Division of Geological \& Geophysical Surveys

MISCELLANEOUS PUBLICATION 129

SURVEY OF GEOLOGY, GEOLOGIC MATERIALS, AND GEOLOGIC HAZARDS IN PROPOSED ACCESS CORRIDORS, ALASKA

This booklet accompanies Miscellaneous Publications 45 through 122 and provides a detailed explanation of the rationale, methodology, and data presented on the maps. It is strongly suggested that this information be available to users of any of the maps in this series.

$\$ 7.00$

2003

THESE MAPS AND REPORT HAVE RECEIVED MODEST TECHNICAL REVIEW AND CONFORM TO THE EDITORIAL STANDARDS OF DGGS.

\author{
Released by \\ STATE OF ALASKA \\ DEPARTMENT OF NATURAL RESOURCES \\ Division of Geological \& Geophysical Surveys \\ 3354 College Rd.
}

Fairbanks, Alaska 99709-3707 


\section{Survey of Geology, Geologic Materials, and Geologic Hazards in Proposed ACCESS CORRIDORS IN ALASKA}

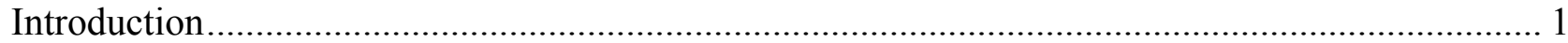

Rationale

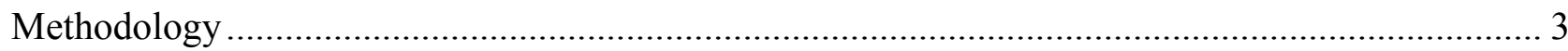

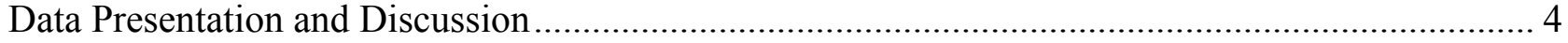

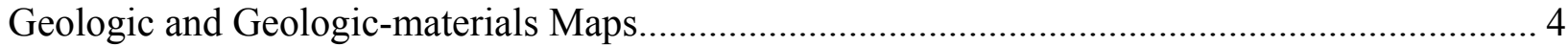

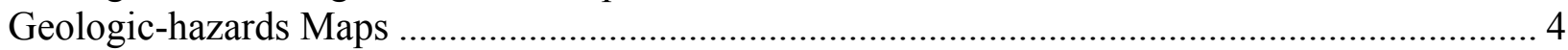

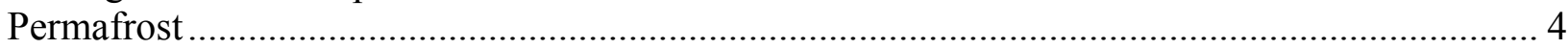

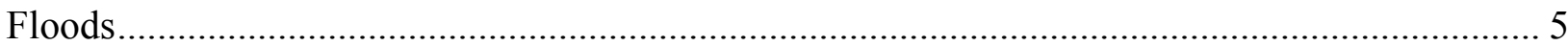

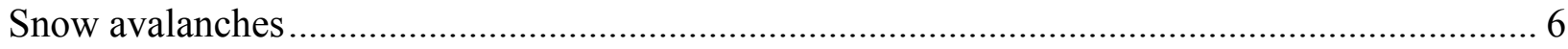

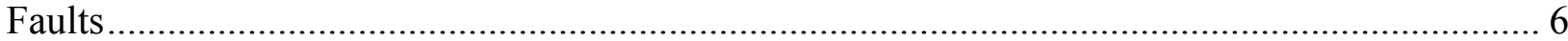

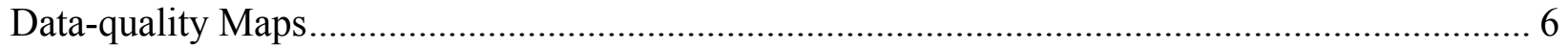

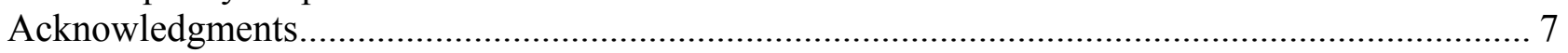

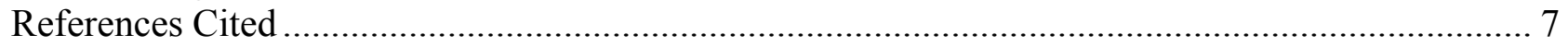

FIGURES

Figure 1. Index map of proposed transportation corridors in Alaska that were evaluated for geology, geologic materials, and geologic hazards.................................................................. 9

Figure 2. Work plan for geologic evaluation of proposed access routes in Alaska......................... 10

Figure 3. Derivation of geologic-hazards maps during evaluation of proposed access routes in

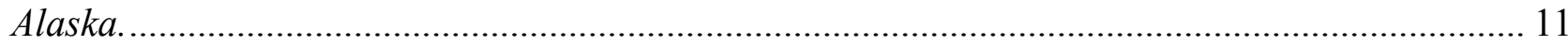

TABLES

Table 1. Relations between materials classes, dominant landforms, and geologic units in geologic

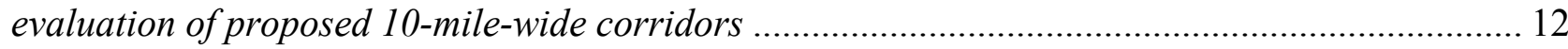

Table 2. Genetic relations of geologic deposits and hazardous processes...................................... 15

Table 3. Genetic relations of geologic-materials classes and hazardous processes ........................ 17

Table 4. Zones of permafrost identified during corridor evaluation .............................................. 19

Table 5. Distribution of permafrost zones by quadrangle crossed by proposed 10-mile-wide

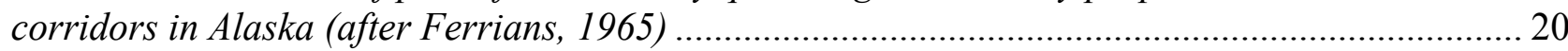

Table 6. Distribution of stream drainages subject to periodic outburst floods in 10-mile-wide corridors containing proposed access routes (Post and Mayo, 1971) .......................................... 21 Table 7. Distribution of stream drainages subject to seasonal icings (naleds) in proposed 10-milewide corridors containing proposed access routes (Dean, 1984a). Numerous small, unnamed

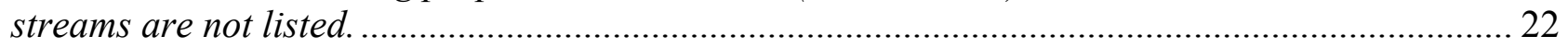

Table 8. Criteria of classes of fault activity used in geologic evaluation of proposed access corridors in Alaska (modified from Howard and others, 1978) ................................................... 25

Table 9. Criteria for data-quality classes used during geologic evaluation of proposed access

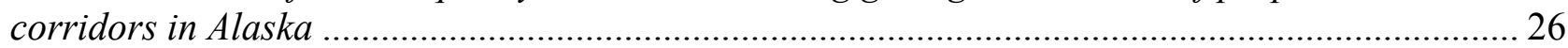
APPENDICES

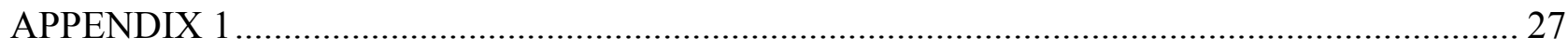

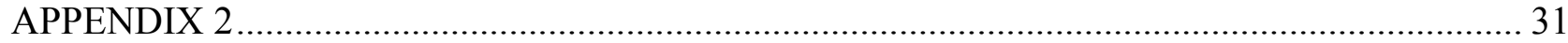

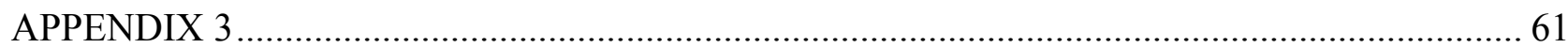

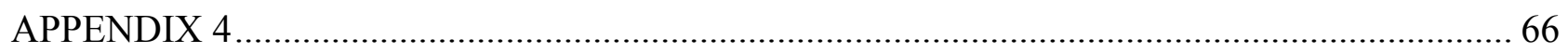




\title{
Survey of Geology, Geologic Materials, and Geologic Hazards in Proposed ACCESS CORRIDORS IN ALASKA
}

\author{
by \\ Richard D. Reger ${ }^{1}$, DeAnne S. P. Stevens ${ }^{2}$, Gina R. Cruse $^{3}$, and Harold R. Livingston ${ }^{4}$
}

\section{INTRODUCTION}

Access to Alaska's mineral lands is a strategic issue for the mineral industry and the state and federal governments. During previous campaigns undertaken by the state of Alaska to choose statehood entitlement lands, many potential access corridors were identified and linked in a conceptual long-range transportation grid. This grid is the basis for much of the state's current transportation planning and is consulted when considering access to new mineral discoveries. In 1992, the Alaska Division of Geological \& Geophysical Surveys (DGGS) compiled a series of 1:250,000-scale digital geologic maps for these transportation corridors from compilations of previously published and unpublished reports, and interpretations of aerial photographs and satellite imagery. The 10-mile-wide corridors straddling proposed access routes were compiled, by quadrangle, so that 1-mile-wide access corridors connecting strategically important centers of population, ports, pinch points, and resource-rich lands could ultimately be located to accommodate favorable terrain and avoid natural hazards and so that available geologic-materials resources could also be selected.

The corridor maps were used for planning during the State land selection process but were never formally released. By 1999 it became apparent that the digital map files were no longer useable because they were made for a plotter technology that was no longer supported. Thus, these maps were no longer available to policy makers, mineral explorationists, and engineers who have need of the information that they portray. In 2001, DGGS instituted a project to formally release the map products and data compiled during the previous study in order to provide public access to geologic, geologic-materials, and engineering-geologic information that can affect decisions about transportation options for mineral development and other diverse enterprises.

The goals of the 1992 DGGS corridor project were: (1) to prepare 1:250,000-scale maps of the geology, geologic materials, and geologic hazards for 10-mile-wide corridors containing proposed access routes and traversing 78 quadrangles (fig. 1); (2) to establish the quality of the data from which these maps were prepared; and (3) to document the results of the study. This report presents the results of those previous efforts, and provides a summary overview to accompany the re-

\footnotetext{
${ }^{1}$ Retired from Engineering Geology Section, Division of Geological \& Geophysical Surveys, 3354 College Rd., Fairbanks, Alaska 99709-3707.

${ }^{2}$ Engineering Geology Section, Division of Geological \& Geophysical Surveys, 3354 College Rd., Fairbanks, Alaska 99709-3707. Deanne_Stevens@dnr.state.ak.us

${ }^{3}$ Employed by Division of Geological \& Geophysical Surveys at the time of original data compilation. Current contact Gina.Graham@alyeska-pipeline.com.

${ }^{4}$ Retired from Geology Section, Design and Construction Division, Department of Transportation and Public Facilities, Northern Regional Office, 2301 Peger Rd., Fairbanks, Alaska 99707.
} 
drafted, single-quadrangle digital map suites. The user should note that the only changes made to the original 1992 products are to the digital data files and map cartography; none of the reference information has been updated to reflect subsequent mapping in the quadrangles traversed by the proposed access corridors.

\section{RATIONALE}

Geologic maps portray the general distribution of geologic units at the surface of the Earth. These maps are prepared by geologists during studies of exposed rocks and sediments or landforms that result from the presence or placement of rocks and sediments. Field and laboratory studies of materials comprising geologic units and landforms in Alaska demonstrate that each geologic unit or landform has a definite composition or range of composition wherever that feature is present (table 1) (Kreig and Reger, 1976, 1982). Thus, we derived a series of geologic-materials maps from geologic maps or by interpreting landforms (in 14 quadrangles where map information is inadequate $^{5}$ (map a). Our geologic-materials maps are generalized. They do not attempt to show exact locations of specific materials but are intended to indicate general areas that deserve consideration for certain materials and to eliminate other general areas from consideration for these materials. Local variations are common, especially near unit boundaries. Precise economic evaluations of specific deposits as sources of construction materials will require detailed examination of each deposit, including areal extent, volume, grain-size variation, thickness of overburden, thermal state of the ground, and depth to water table as well as logistical factors and demand.

Data-quality maps (map b) present our best judgment of the reliability of available geologic information based on several factors, including the type and date of topographic maps used as the base upon which the data were initially plotted, the presence or absence of published or unpublished information, the type and date of the geologic report, who prepared the report (specialist vs. nonspecialist), the complexity of the area mapped, the amount of field verification, and (where we interpreted aerial photographs or satellite imagery to produce geologic and geologic-materials maps) the date and scale of the aerial photography and satellite imagery.

For the 64 quadrangles where geologic mapping is reliable ${ }^{6}$, geologic-hazards maps were derived directly from geologic maps because (1) the processes that produced the geologic deposits also threaten property and human lives and activities or (2) conditions in the deposits are hazardous (table 2) (map c, sheet 1, if applicable). In the 14 quadrangles where geologic mapping is not reliable $^{7}$, derivative geologic-hazards maps were developed from geologic-materials maps and contain complex hazards units, including several combined hazardous processes (table 3) (map c, sheet 1, if applicable).

\footnotetext{
${ }^{5}$ Landforms were interpreted for Baird Mountains, Bradfield Canal, Dillingham, Iditarod, Juneau, Lake Clark, Lime Hills, Naknek, Noatak, Ruby, Sagavanirktok, Sleetmute, Taku River, and Taylor Mountains quadrangles because geologic maps were not available.

${ }^{6}$ During project reliable geologic mapping did not exist for the Baird Mountains, Bradfield Canal, Dillingham, Iditarod, Juneau, Lake Clark, Lime Hills, Naknek, Noatak, Ruby, Sagavanirktok, Sleetmute, Taku River, and Taylor Mountains quadrangles.

${ }^{7}$ Ibid.
} 
Non-derivative (additional) geologic-hazards maps, which illustrate areas of significant potential for snow avalanches and drainages that are subject to stream icings and outburst floods, were developed from an interpretation of 1:250,000-scale topographic maps or from information published in the technical literature (map c, sheet 2). Source slopes for snow avalanches are inferred from slope angles, which are reflected in the spacing of contours on 1:250,000-scale topographic maps. We use an inclination of $30^{\circ}$ (58 percent) as a lower threshold for slopes of moderate to high potential for producing snow avalanches because this is generally the shallowest slope for initiating dry-snow and slab avalanches (Perla and Martinelli, 1976). However, wet-snow avalanches are initiated on slopes as gentle as $15^{\circ}(27$ percent $)$ and factors (like surface roughness, wind drifting, temperature gradients, snow metamorphism, water content, and snow creep) that affect the failure of snow packs are complex (Perla and Martinelli, 1975). Similarly, runout distances, directions, and effects vary considerably and are difficult to predict without detailed studies of past avalanche paths (Martinelli, 1974; Burrows and Burrows, 1976; Mears, 1976).

Although they are the source data for many of the aforementioned maps, the geologic maps are discussed last because they are not available for all 78 quadrangles that this report summarizes. Geologic maps for the 64 quadrangles where geologic mapping is reliable portray simplified geology compiled from published sources (map d, if applicable). Age distinctions were eliminated and geologic units combined based on similar lithologic character, which could then be used to derive the construction-materials and geologic-hazards maps.

\section{METHODOLOGY}

After centerlines of proposed access routes were located through most of Alaska, corridor limits were established at 5 miles on either side of the centerlines (fig. 2). Geologic information on these corridors was assembled, reviewed, and compiled from published and unpublished reports, aerial photographs, and satellite imagery.

In quadrangles where the published or unpublished information is reliable, maps of surficial and bedrock geology were traced and then digitized into the DGGS geographic information system (GIS), where the data could be stored, retrieved, manipulated, and printed (fig. 2). Then geologicmaterials and geologic-hazards maps were derived from the geologic maps and printed.

Where the published or unpublished information is unreliable or nonexistent, maps of surficial geology and unconsolidated geologic materials were produced by interpreting 1,097 false-color, infrared aerial photographs (Baird Mountains, Barter Island, Bettles, Bradfield Canal, Dillingham, Holy Cross, Hughes, Iditarod, Juneau, Lake Clark, Lime Hills, Melozitna, Naknek, Noatak, Ruby, Sagavanirktok, Sleetmute, Taku River, and Taylor Mountains quadrangles)(app. 1), and one scene of 1:250,000-scale, enhanced and rectified satellite imagery (scene 10756-21121 dated 8-18-74) was interpreted to prepare a geologic-materials map of the Port Moller Quadrangle (fig. 2).

Geologic-hazards maps were prepared only for 10-mile-wide corridors, except that active fault zones are delineated throughout the 1:250,000-scale quadrangles traversed by the corridors, and streams that are susceptible to outburst floods are identified outside the corridor in the Cordova Quadrangle. Sources of information needed to prepare geologic-hazards maps include (1) geologic 
and geologic-materials maps previously developed during this study, (2) 1:60,000-scale false-color, infrared aerial photographs, (3) supplemental technical literature, and (4) 1:250,000-scale topographic maps (fig. 3).

\section{DATA PRESENTATION AND DiscUSSION}

The primary products of the DGGS Corridor Evaluation Project are the 376 1:250,000-scale geologic-materials, data-quality, derivative geologic-hazards, non-derivative (additional) geologichazards, and geologic maps that accompany this report.

\section{Geologic and Geologic-materials Maps}

A total of 190 sources of technical information were consulted during our extensive compilation of data on corridor geology and geologic materials. These references are alphabetically listed by quadrangle in appendix 2.

\section{Geologic-hazards Maps}

A total of 83 sources of information on geologic hazards were consulted during our research. In addition to identifying potentially active faults and drainages affected by thick stream icings and outburst floods on non-derivative (additional) hazards maps, we established 13 classes of geologic hazards on derivative-hazards maps (table 3). In general, our main concerns in lowland areas are natural hazards related to a lack or loss of bearing (shear) strength (saturated, organic-rich swamp deposits and thawing of ice-rich permafrost) and flooding. In highlands, mass movements are locally a serious concern. Based on topography, we identified terrain where there generally is moderate to high potential for snow avalanches, including probable runout zones. Local, unevaluated factors affecting other forms of mass movement (rock avalanches, landslides, and debris flows) include textures of sediments, bedrock structures, and water content. Faulting and associated earthquakes produce sudden displacements by shaking and impacts and by widespread liquefaction of foundations soils in both lowlands and highlands.

\section{Permafrost}

Permafrost is rock or soil, or both, that has natural temperatures that are consistently less than $0^{\circ} \mathrm{C}$ for 2 years or more (Péwé, 1982). This perennially frozen ground seriously affects many human activities in Alaska, especially the construction and maintenance of roads, railroads, airfields, bridges, buildings, dams, sewers, oil and gas pipelines, and communication and utility systems. Fundamental problems include: (1) thawing of ice-rich foundation materials beneath roads, airfields, and agricultural fields; (2) ground subsidence under heated structures; (3) intensification of frost action due to impeded drainage; and (4) freezing of buried sewer, water, and oil lines (Péwé, 1982). 
In mountainous terrain, which is typically complex and rugged, great differences in elevation, materials, snowfall, vegetation cover, soil moisture, and insolation (solar radiation received at the ground surface) result in considerable variation in the temperature, ice content, thickness, and distribution of permafrost. In uplands with less relief and in lowlands, temperature, thickness, and distribution of permafrost are less variable.

Local permafrost distribution is extremely complex because it depends on complicated microclimatic factors, including aspect, slope angle, temperature at the ground surface, material type, and ground insulation. Therefore, for the purposes of this report, we only identify the general distribution of permafrost in each 1:250,000-scale quadrangle through which 10-mile-wide corridors pass. Six zones of general distribution include (1) continuous, (2) transitional from continuous to discontinuous, (3) discontinuous, (4) transitional from discontinuous to sporadic, (5) sporadic, and (6) no permafrost (table 4).

Among the quadrangles crossed by the 10-mile-wide corridors that we evaluated, only 11 quadrangles (14.1 percent) do not include at least some perennially frozen ground (table 5).

\section{Floods}

Low-relief, planar surfaces (floodplains) bordering streams are normally inundated, at least in part, each year by floods during spring melting of seasonal snow cover and during sustained or heavy rainfall. However, special conditions exist in Alaska that periodically produce exceptional flooding.

Sudden drainage of the waters impounded in mountain valleys blocked by glaciers produces brief floods characterized by stream stages well above normal flood levels (Post and Mayo, 1971). These outburst floods or jökulhlaups are not necessarily annual events, although they occur annually in some drainages. Impounded waters break out of their basins when they overflow low thresholds on the ice dam and cut rapidly down through the ice or when waters percolating under the glacier buoy it up enough to start subglacial drainage. Outburst floods are particularly hazardous during middle to late winter when stream channels are choked by seasonal ice and ice-jam flooding exacerbates the problem. In Alaska, significant outburst floods are limited to mountainous areas from the Alaska Range south (Post and Mayo, 1971). During our survey, we located 34 drainages that are susceptible to outburst flooding in 10 of 78 quadrangles (12.8 percent) traversed by 10 -mile-wide corridors containing proposed access routes (table 6). In our study we take the conservative approach that outbursts from glacier-blocked lakes in headwater reaches affect the entire drainage, although bank storage in downstream reaches generally attenuates the abrupt rise in stream-stage level normally associated with jökulhlaups.

Each winter, braided streams and shallow reaches of other streams freeze to the bottom, producing exceptionally thick stream icings (naleds) by the freezing of successive thin sheets of overflow water forced by hydraulic stress up through contraction cracks in the seasonal ice cover (Sloan and others, 1976). Similar seasonal-icing sheets develop on slopes below springs. The gradual buildup of seasonal-ice sheets slowly inundates facilities and other property sited above the normal highwater level of nearby streams (Nelson, 1978; Péwé, 1982). Seasonal icings are widely distributed in Alaska but are more common in the northern half of the state (Sloan and others, 1976; Harden and 
others, 1977; Dean, 1984a, b). During our survey, we located 193 streams and numerous additional unnamed tributaries subject to seasonal icings in 55 of 78 quadrangles ( 70.5 percent) traversed by 10-mile-wide corridors (table 7).

Snow avalanches

Snow avalanches are sliding and turbulently flowing masses of snow, air, water, and debris that are capable of destroying people, structures, and property because of their spontaneity and the considerable forces that they generate (Mellor, 1968). In our brief reconnaissance, we have identified areas that we believe have moderate to high potential for snow avalanches, regardless of type, including estimated runout zones in 38 of 78 quadrangles (48.7 percent).

\section{Faults}

Faults are breaks in the Earth's crust along which significant movement has occurred. Sudden displacements along faults generate earthquakes that can be hazardous to people, structures, and property because of falling objects, slope failures, ground shaking and cracking, and liquefaction effects (Hansen, 1966). Hazards of infrequent, strong earthquakes (like the March 27, 1964 Prince William Sound earthquake) are regional in scale, but consideration of potential regional effects during future earthquakes is beyond the scope of our study.

Realizing that the Aleutian megathrust, which is not a surface fault, is the most extensive and dangerous source of future earthquakes in Alaska (Plafker, 1969), for the purposes of our evaluation of proposed access routes, we focus on more local influences of faulting by identifying and mapping surface faults in those quadrangles traversed by the 10-mile-wide corridors being evaluated (app. 3). Faults are not evaluated in quadrangles not crossed by corridors, even though these structural breaks may generate significant earthquakes there. Depending on when the last movement occurred along them, surface faults are designated as definitely active, probably active, or possibly active (table 8); inactive faults are not considered to have engineering significance. Where adequate data are available, we identify fault segments with different levels of activity. However, where data are not sufficiently detailed to separate segments, we take a conservative approach that the most active fault segment is the best measure of potential future displacements along the entire fault.

\section{Data-quality Maps}

Each source of map information on geology and geologic materials was evaluated for data quality (app. 4). Five classes of data quality range from very good to poor (table 9). 


\section{ACKNOWLEDGMENTS}

The concept of identifying and evaluating access corridors through most of Alaska for the purposes of land selection was first championed by Jerry Brossia, State Pipeline Coordinator, who got the ball rolling in Department of Natural Resources. Richard "OD" Odsather, State Pipeline Coordinator's Office, became our very capable project leader in July 1991 and soon organized the Corridor Selection Steering Committee, of which our team was a small part. OD personally spent much time, thought, and effort in the complicated process of locating routes for us to evaluate. During this process, the knowledgeable counsel of Edwin "Rocky" Rhoads was invaluable. Both Jerry and OD supported us with enthusiasm, timely arm-twisting, and vital funds when we needed them the most.

Our evaluation team received considerable timely help with digitizing from Nori Bowman, Kathy Campbell, and John Roe. Richard Clement and Gail March helped immeasurably with training and supervision of everyone working with the new DGGS geographic information system.

Florence R. Weber kindly loaned us her personal copies of USGS reports that we could not locate in local libraries.

Robin Smith, intern in the Engineering Geology Section, DGGS, updated the 362 corridor coverages, and underlaid quadrangle topography base maps to create the finished product in 2002.

Without the generosity and support of all these folks, our products would have been inferior and our deadlines not met.

\section{REFERENCES CITED}

Burrows, C.J., and Burrows, V.L., 1976, Procedures for the study of snow avalanche chronology using growth layers of woody plants: Institute of Arctic and Alpine Research Occasional Paper 23, 54 p.

Dean, K.G., 1984a, Stream-icing zones in Alaska: Alaska Division of Geological \& Geophysical Surveys Report of Investigations 84-16, 20 p., scale 1:250,000, 102 sheets.

1984b, Remote sensing in the north: Alaskan stream icings: The Northern Engineer, v. 16, no. 1, p. 4-7.

Ferrians, O.J., Jr., 1965, Permafrost map of Alaska: U.S. Geological Survey Miscellaneous Geologic Investigations Map I-445, scale 1:2,500,000, 1 sheet.

Hansen, W.R., 1966, Effects of the earthquake of March 27, 1964, at Anchorage, Alaska: U.S. Geological Survey Professional Paper 542-F, 68 p, scale 1:2400, 1 sheet.

Harden, Deborah, Barnes, Peter, and Reimnitz, Erk, 1977, Distribution and character of naleds in northeastern Alaska: Arctic, v. 30, no. 1, p. 28-40. 
Howard, K.A., Aaron, J.M., Brabb, E.E., Brock, M.R., Gower, H.D., Hunt, S.J., Milton, D.J., Muehlberger, W.R., Nakata, J.K., Plafker, G., Prowell, D.C., Wallace, R.E., and Witkind, I.J., 1978, Preliminary map of young faults in the United States as a guide to possible fault activity: U.S. Geological Survey Miscellaneous Field Studies Map MF-916, scales $1: 5,000,000$ and $1: 750,000,2$ sheets.

Kreig, R.A., and Reger, R.D., 1976, Preconstruction terrain evaluation for the trans-Alaska pipeline project, in Coates, D.R., ed., Geomorphology and Engineering, Stroudsburg, Dowden, Hutchinson, and Ross, p. 55-76.

1982, Air-photo analysis and summary of landform soil properties along the route of the trans-Alaska pipeline system: Alaska Division of Geological \& Geophysical Surveys Geologic Report 66, 149 p.

Martinelli, M., 1974, Snow avalanche sites, their identification and evaluation: U.S. Forest Service Agriculture Information Bulletin 360, 27 p.

Mears, A.I., 1976, Guidelines and methods for detailed snow avalanche hazard investigations in Colorado: Colorado Geological Survey Bulletin 38, 125 p.

Mellor, Malcolm, 1968, Avalanches: U.S. Army Cold Regions Research and Engineering Laboratory Cold Regions Science and Engineering Monograph III-A3d, 215 p.

Nelson, G.L., 1978, Hydrologic information for land-use planning, Fairbanks vicinity, Alaska: U.S. Geological Survey Open-file Report 78-959, 47 p.

Perla, R.I., and Martinelli, M., Jr., 1976, Avalanche handbook: U.S. Forest Service Agriculture Handbook 489, 238 p.

Péwé, T.L., 1982, Geologic hazards of the Fairbanks area, Alaska: Alaska Division of Geological \& Geophysical Surveys Special Report 15, 109 p.

Plafker, George, 1969, Tectonics of the March 27, 1964, Alaska earthquake: U.S. Geological Survey Professional Paper 543-I, 74 p., scales 1:500,000 and 1:2,000,000, 2 sheets.

Post, Austin, and Mayo, L.R., 1971, Glacier dammed lakes and outburst floods in Alaska: U.S. Geological Survey Hydrologic Atlas HA-455, 9 p., scale 1:1,000,000, 3 sheets.

Sloan, C.E., Zenone, Chester, and Mayo, L.R., 1976, Icings along the trans-Alaska pipeline route: U.S. Geological Survey Professional Paper 979, 31 p. 
EXPLANATION

Centerline of proposed transportation corrido 1:250,000-scale U.S.G.S. quadrangle boundary

Existing road

- Towns with populations greater than 1,000 people

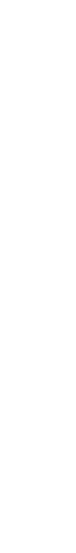
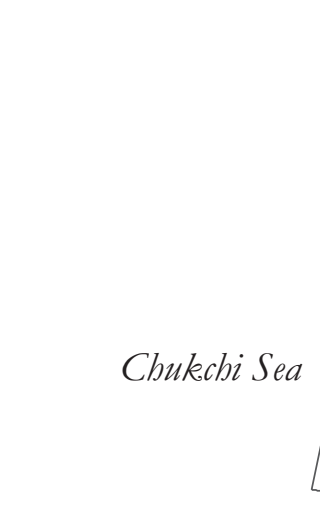

Arctic Ocean
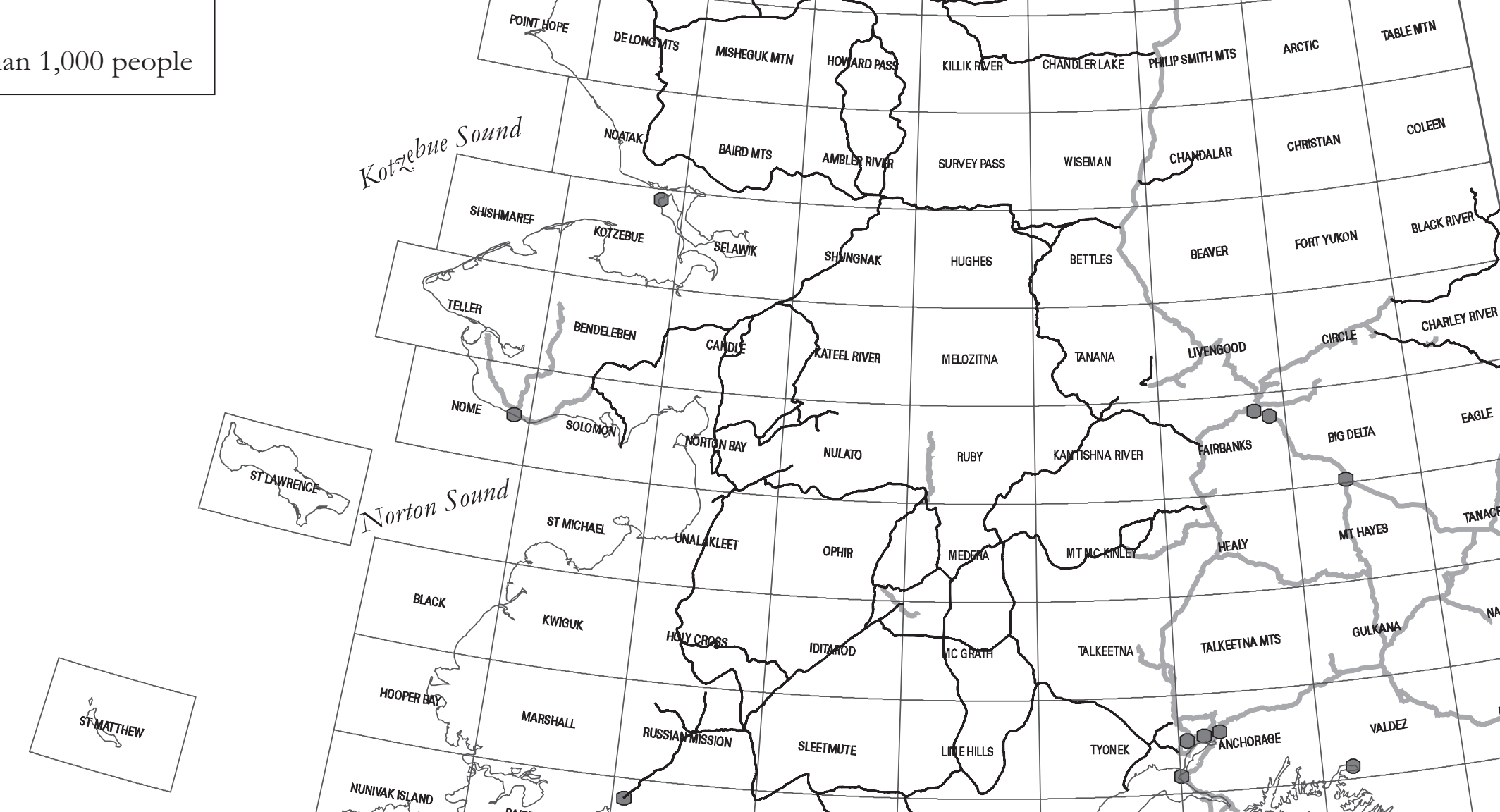

\section{C \\ A}

A
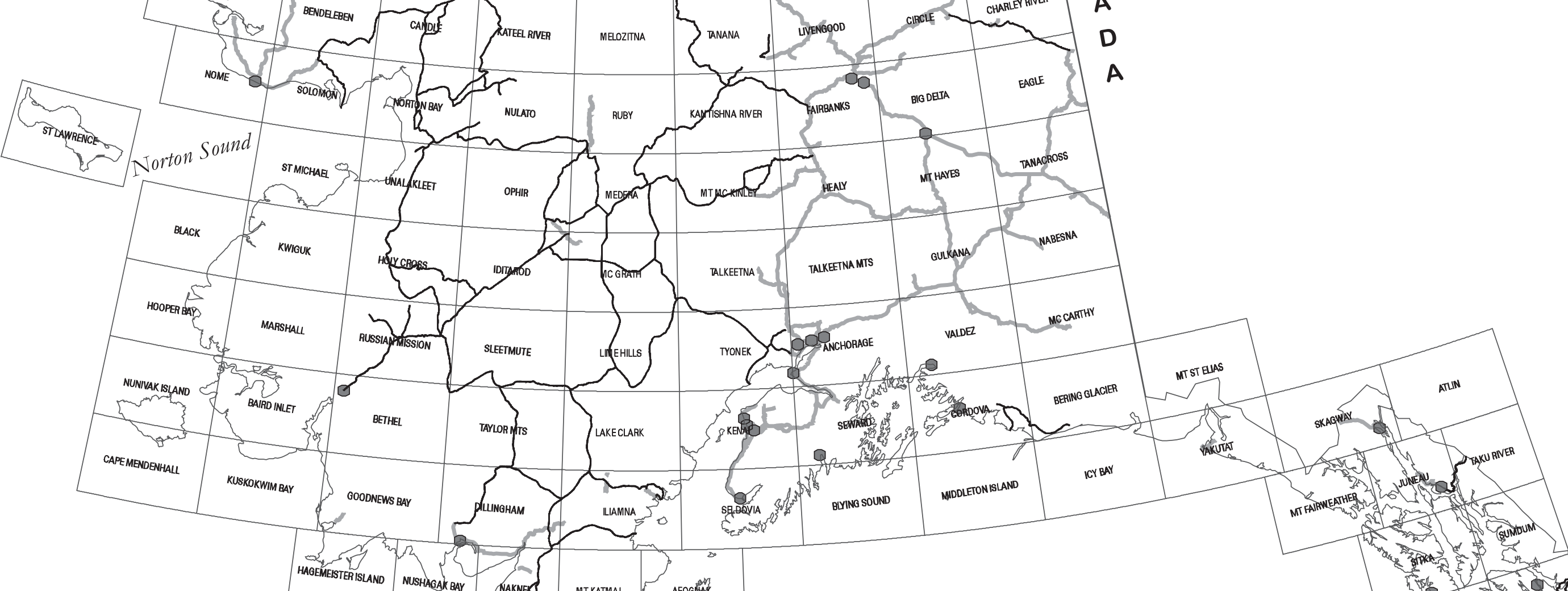

$$
\text { Problor ISLANDS }
$$

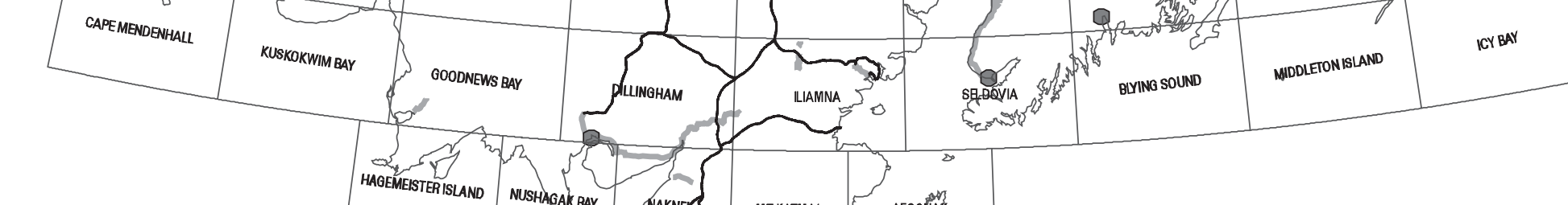

Gulf of Alaska

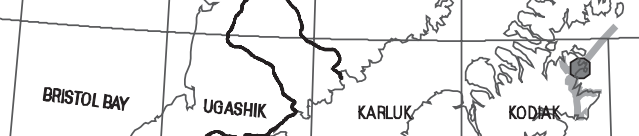

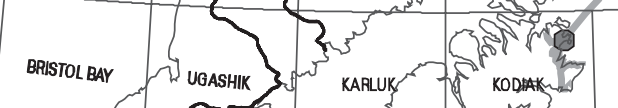

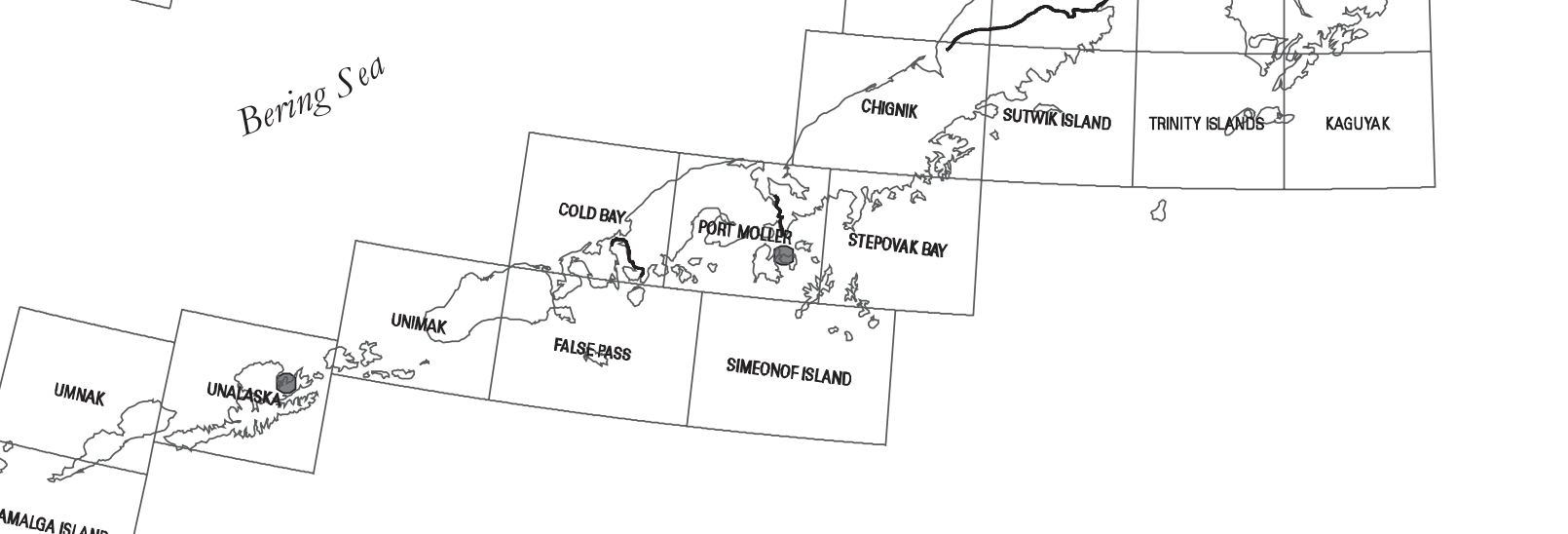

$250 \quad 500$ Kilometers

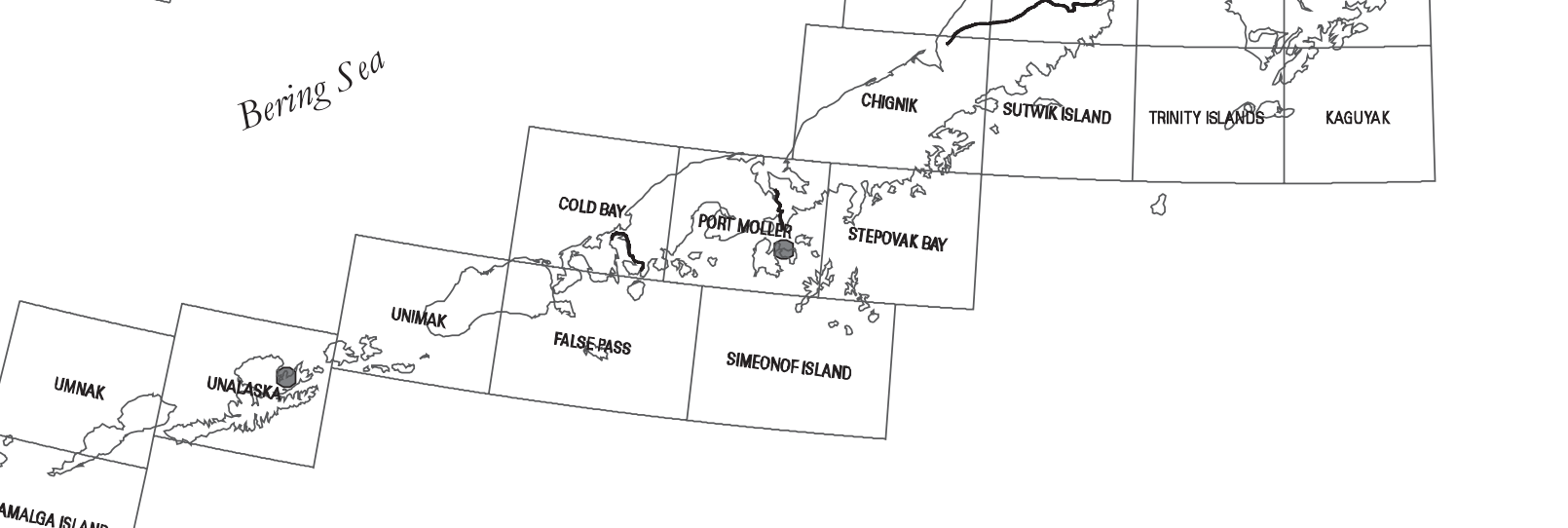

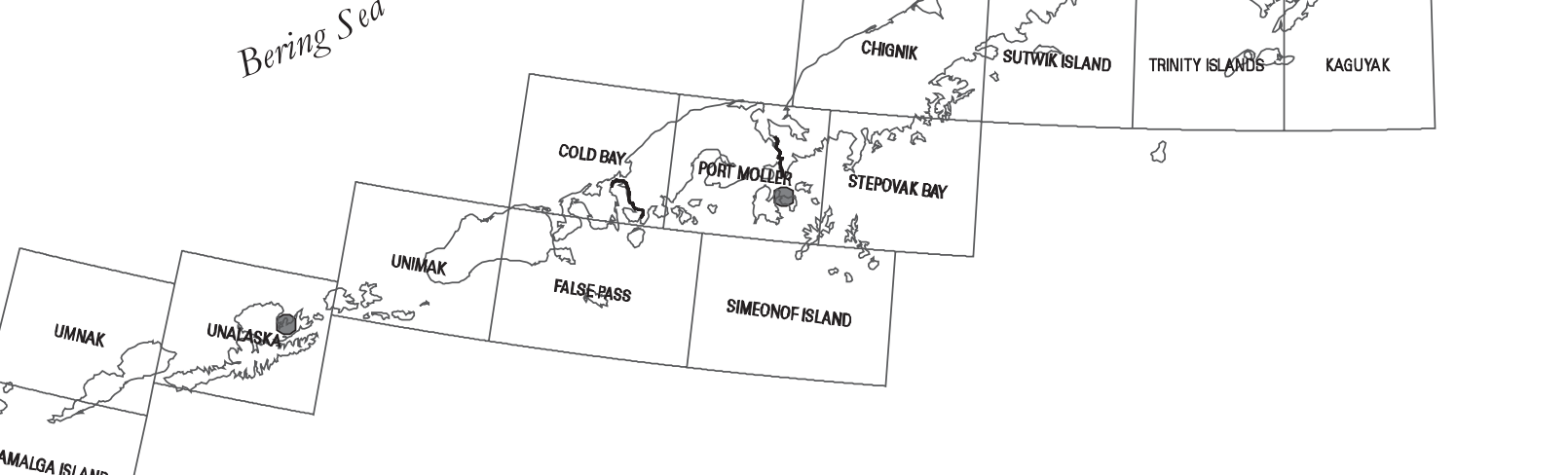

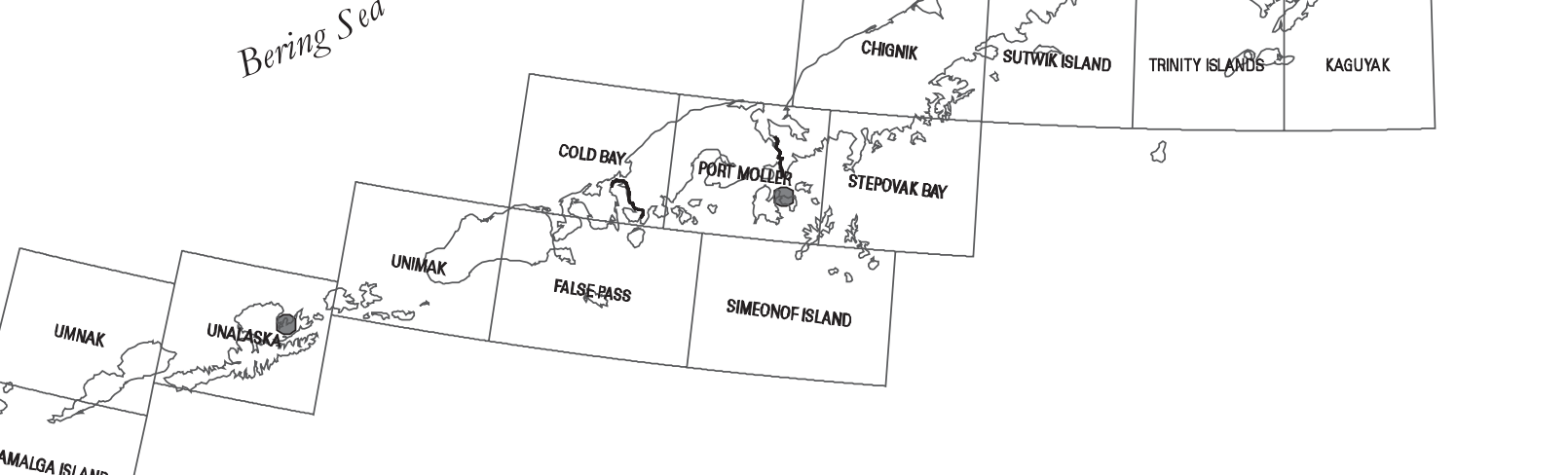

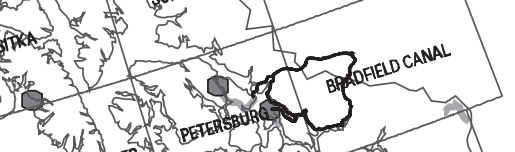

5 s

porf

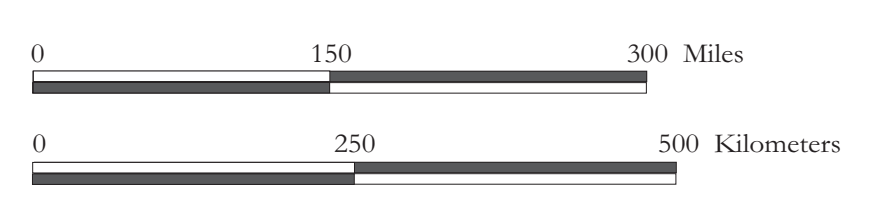

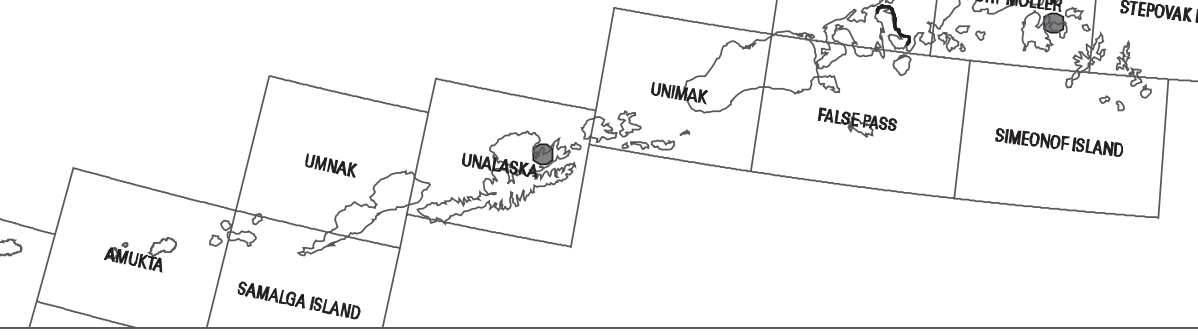

Figure 1. Index map of proposed transportation corridors in Alaska that were evaluated for geology, geologic materials, and geologic hazards. 


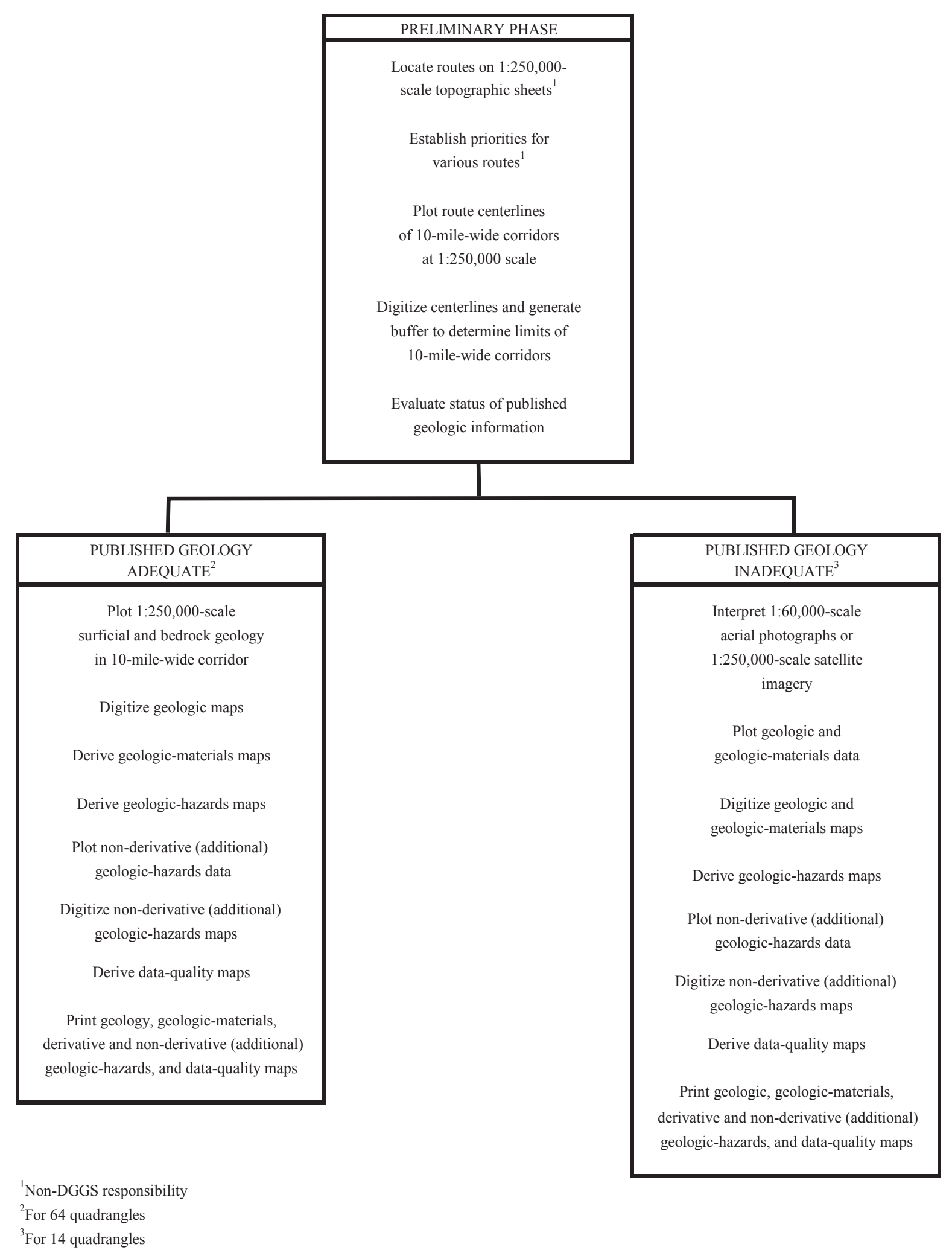

Figure 2. Work plan for geologic evaluation of proposed access routes in Alaska. 


\section{DATA SOURCES ACTION PRODUCT}
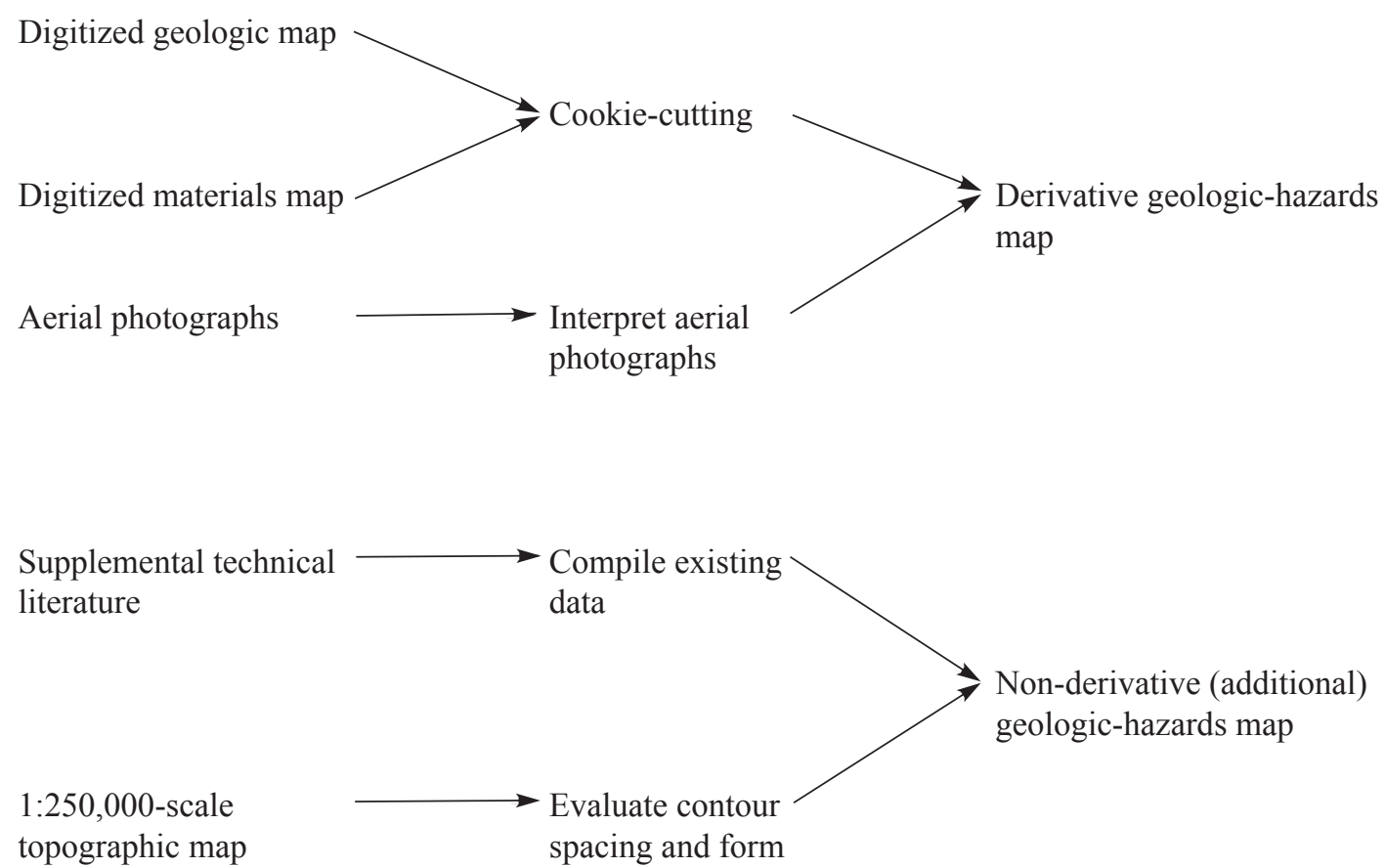

Figure 3. Derivation of geologic-hazards maps during evaluation of proposed access routes in Alaska. 
Table 1. Relations between materials classes, dominant landforms, and geologic units in geologic evaluation of proposed 10-mile-wide corridors

\begin{tabular}{|c|c|c|c|c|}
\hline $\begin{array}{c}\text { Materials } \\
\text { unit }\end{array}$ & Composition & Primary products & Component landforms & Geologic unit \\
\hline SA & $\begin{array}{l}\text { Chiefly (estimated 80\%) sand } \\
\text { Includes SW and SP (Unified } \\
\text { Soil Classification) }\end{array}$ & Unclassified fine fills & $\begin{array}{l}\text { Eolian sand dune, sheet, dune } \\
\text { complex } \\
\text { Retransported sand fan, } \\
\text { apron, sheet }\end{array}$ & $\begin{array}{l}\text { Eolian sand (Qes) } \\
\text { Undifferentiated eolian } \\
\text { sand and silt (Qe) } \\
\text { Reworked eolian deposits } \\
\text { (Qer) }\end{array}$ \\
\hline
\end{tabular}




\begin{tabular}{|c|c|c|c|c|}
\hline $\begin{array}{l}\text { Materials } \\
\text { unit }\end{array}$ & Composition & Primary products & Component landforms & Geologic unit \\
\hline OR & $\begin{array}{l}\text { Chiefly organic materials } \\
\text { Estimated }>50 \% \text { peat, organic } \\
\text { sand, or organic silt } \\
\text { Includes Pt (Unified Soil } \\
\text { Classification) }\end{array}$ & Unclassified fine fills & $\begin{array}{l}\text { Thaw-lake basin } \\
\text { Swamp }\end{array}$ & $\begin{array}{l}\text { Thaw-lake deposits (Qtl) } \\
\text { Swamp deposits (Qs) }\end{array}$ \\
\hline $\mathrm{BV}$ & $\begin{array}{l}\text { Medium-jointed, fine-grained } \\
\text { igneous rocks and their } \\
\text { metamorphic equivalents } \\
\text { May be deeply weathered }\end{array}$ & $\begin{array}{l}\text { Riprap, drain rock } \\
\text { Crushed rock } \\
\text { Unclassified fills }\end{array}$ & $\begin{array}{l}\text { Volcanic flow rock outcrop } \\
\text { Welded tuff outcrop } \\
\text { Greenstone outcrop }\end{array}$ & $\begin{array}{l}\text { Volcanic rocks }(\mathrm{Biv}) \\
\text { Volcanic rocks }(\mathrm{Biv}) \\
\text { Greenstone }(\mathrm{Bmg})\end{array}$ \\
\hline
\end{tabular}




\begin{tabular}{|c|c|c|c|c|}
\hline $\begin{array}{c}\text { Materials } \\
\text { unit }\end{array}$ & Composition & Primary products & Component landforms & Geologic unit \\
\hline $\mathrm{BO}$ & $\begin{array}{l}\text { All other lithologies } \\
\text { May be deeply weathered }\end{array}$ & Unclassified fills & $\begin{array}{l}\text { Outcrops of all bedrock } \\
\text { classes not included in } \\
\text { above groups }\end{array}$ & $\begin{array}{l}\text { Chert (Bsch), } \\
\text { Shale (Bsh), } \\
\text { Siltstone (Bsi), } \\
\text { Schist, serpentine, } \\
\text { phyllite, fine-grained } \\
\text { gneiss (Bms) }\end{array}$ \\
\hline $\mathrm{BU}$ & $\begin{array}{l}\text { Undifferentiated, unknown } \\
\text { bedrock } \\
\text { May be deeply weathered }\end{array}$ & Unclassified fills & Undifferentiated bedrock & $\begin{array}{l}\text { Undifferentiated igneous } \\
\text { rock (Biu), } \\
\text { Undifferentiated } \\
\text { sedimentary rock }(\mathrm{Bsu}) \text {, } \\
\text { Undifferentiated } \\
\text { metamorphic rock } \\
(\mathrm{Bmm}), \\
\text { Undifferentiated, } \\
\text { unknown rock }(\mathrm{Bu})\end{array}$ \\
\hline
\end{tabular}


Table 2. Genetic relations of geologic deposits and hazardous processes

\begin{tabular}{|c|c|c|c|c|c|}
\hline \multirow{2}{*}{$\begin{array}{l}\text { SURFICIAL } \\
\text { DEPOSIT }\end{array}$} & \multicolumn{5}{|c|}{ TYPE OF GEOLOGIC HAZARD } \\
\hline & $\begin{array}{l}\text { Rapid mass } \\
\text { movements }\end{array}$ & $\begin{array}{l}\text { Significant } \\
\text { liquefaction } \\
\text { potential }^{2}\end{array}$ & $\begin{array}{l}\text { Significant ground- } \\
\text { ice content }\end{array}$ & Seasonal flooding $^{4}$ & $\begin{array}{c}\text { Snow } \\
\text { avalanching }^{5}\end{array}$ \\
\hline $\begin{array}{l}\text { Undifferentiated alluvium } \\
\text { (Qa) }\end{array}$ & - & $\mathrm{X}$ & - & $\mathrm{X}$ & - \\
\hline Alluvial-fan deposits (Qaf) & $\mathrm{X}$ & - & - & $\mathrm{X}$ & $\mathrm{X}$ \\
\hline Terrace alluvium (Qat) & - & - & X (SM only) & - & - \\
\hline Placer-mine tailings (Qmt) & - & - & - & - & - \\
\hline Fine-grained alluvium (Qsm) & - & $\mathrm{X}$ & $\mathrm{X}$ & $\mathrm{X}$ & - \\
\hline $\begin{array}{l}\text { Undifferentiated colluvium } \\
\text { (Qc) }\end{array}$ & - & - & $\mathrm{X}$ & - & - \\
\hline Debris-flow deposits (Qcd) & $\mathrm{X}$ & - & - & - & - \\
\hline Landslide deposits (Qcl) & $\mathrm{X}$ & - & - & - & $\mathrm{X}$ \\
\hline Solifluction deposits (Qcs) & - & - & $\mathrm{X}$ & - & - \\
\hline Talus (Qct) & $\mathrm{X}$ & - & $\mathrm{X}$ & - & $\mathrm{X}$ \\
\hline Rock-glacier deposits (Qrg) & $\mathrm{X}$ & - & $\mathrm{X}$ & - & $\mathrm{X}$ \\
\hline $\begin{array}{l}\text { Undifferentiated eolian sand } \\
\text { and silt (Qe) }\end{array}$ & - & - & $X$ & - & - \\
\hline Loess (Qel) & - & - & $\mathrm{X}$ & - & - \\
\hline $\begin{array}{l}\text { Reworked eolian deposits } \\
\text { (Qer) }\end{array}$ & - & $X$ & $\mathrm{X}$ & - & - \\
\hline Dune sand (Qes) & - & - & - & - & - \\
\hline
\end{tabular}

\footnotetext{
${ }^{1}$ Includes landslides, avalanches, and debris flows. Significant potential for displacements and impacts.

${ }^{2}$ Where fine grained (sand and silt), saturated, and thawed, there is significant potential for loss of shear strength during conditions of dynamic loading (shaking).

${ }^{3}$ Where perennially frozen, contains significant pervasive or massive ice, or both. During and after melting, susceptible to excessive and differential settlements and loss of shear strength.

${ }^{4}$ Includes seasonal stream icings.

${ }^{5}$ Includes slushflows during periods of rapid spring melting in Brooks Range and on Seward Peninsula.
} 


\begin{tabular}{|c|c|c|c|c|c|}
\hline \multirow{2}{*}{$\begin{array}{l}\text { SURFICIAL } \\
\text { DEPOSIT }\end{array}$} & \multicolumn{5}{|c|}{ TYPE OF GEOLOGIC HAZARD } \\
\hline & $\begin{array}{l}\text { Rapid mass } \\
\text { movements }\end{array}$ & $\begin{array}{l}\text { Significant } \\
\text { liquefaction } \\
\text { potential }^{2}\end{array}$ & $\begin{array}{l}\text { Significant ground- } \\
\text { ice content }\end{array}$ & Seasonal flooding $^{4}$ & $\begin{array}{c}\text { Snow } \\
\text { avalanching }^{5}\end{array}$ \\
\hline $\begin{array}{l}\text { Undifferentiated lacustrine } \\
\text { deposits (Q1) }\end{array}$ & - & $\mathrm{X}$ & $\mathrm{X}$ & - & - \\
\hline Lake-beach deposits (Qlb) & - & - & - & - & - \\
\hline Fan-delta deposits (Qld) & - & $\mathrm{X}$ & $\mathrm{X}$ & - & - \\
\hline Thaw-lake deposits (Qtl) & - & $\mathrm{X}$ & $\mathrm{X}$ & $\mathrm{X}$ & - \\
\hline $\begin{array}{l}\text { Marine-beach deposits } \\
\text { (Qmb) }\end{array}$ & - & - & - & - & - \\
\hline Estuarine deposits (Qme) & - & $\mathrm{X}$ & $\mathrm{X}$ & $\mathrm{X}$ & - \\
\hline $\begin{array}{l}\text { Deltaic and lagoonal deposits } \\
\text { (Qml) }\end{array}$ & - & $\mathrm{X}$ & $\mathrm{X}$ & $X$ & - \\
\hline Undifferentiated drift (Qd) & - & - & $\mathrm{X}$ & - & - \\
\hline Outwash/inwash (Qo) & - & - & - & $\mathrm{X}$ & - \\
\hline Ice-stagnation deposits (Qis) & - & - & - & - & - \\
\hline Swamp deposits (Qs) & - & - & $\mathrm{X}$ & $\mathrm{X}$ & - \\
\hline $\begin{array}{l}\text { Undifferentiated surficial } \\
\text { deposits }(\mathrm{Qu})\end{array}$ & $?$ & $?$ & $?$ & $?$ & $?$ \\
\hline Glacial ice (Ice) & $X$ & - & - & - & $\mathrm{X}$ \\
\hline
\end{tabular}


Table 3. Genetic relations of geologic-materials classes and hazardous processes

\begin{tabular}{|c|c|c|c|c|c|}
\hline \multirow{2}{*}{$\begin{array}{l}\text { MATERIALS } \\
\text { CLASS }\end{array}$} & \multicolumn{5}{|c|}{ TYPE OF GEOLOGIC HAZARD } \\
\hline & $\begin{array}{l}\text { Rapid mass } \\
\text { movements }\end{array}$ & $\begin{array}{l}\text { Significant } \\
\text { liquefaction } \\
\text { potential }^{2}\end{array}$ & $\begin{array}{l}\text { Significant ground- } \\
\text { ice content }\end{array}$ & Seasonal flooding $^{4}$ & $\begin{array}{c}\text { Snow } \\
\text { avalanching }^{5}\end{array}$ \\
\hline Chiefly sand and gravel (GS) & - & $\mathrm{X}$ & $\mathrm{X}$ & $\mathrm{X}$ & - \\
\hline $\begin{array}{l}\text { Mixed coarse and fine } \\
\text { material }(\mathrm{GM})\end{array}$ & $\mathrm{X}$ & $\mathrm{X}$ & $\mathrm{X}$ & $\mathrm{X}$ & $\mathrm{X}$ \\
\hline Chiefly sand (SA) & - & - & - & - & - \\
\hline Chiefly fine materials (SM) & - & $\mathrm{X}$ & $\mathrm{X}$ & $\mathrm{X}$ & - \\
\hline $\begin{array}{l}\text { Chiefly organic material } \\
\text { (OR) }\end{array}$ & - & $\mathrm{X}$ & $\mathrm{X}$ & $\mathrm{X}$ & - \\
\hline $\begin{array}{l}\text { Coarse-jointed, coarse- } \\
\text { grained igneous lithologies } \\
(\mathrm{BG})^{6}\end{array}$ & - & - & - & - & - \\
\hline $\begin{array}{l}\text { Medium-jointed, fine- } \\
\text { grained igneous lithologies } \\
(\mathrm{BV})^{6}\end{array}$ & - & - & - & - & - \\
\hline $\begin{array}{l}\text { Medium-jointed, fine- to } \\
\text { coarse-grained sedimentary } \\
\text { carbonate rocks and their } \\
\text { metamorphic equivalents } \\
\text { (BC) }{ }^{6}\end{array}$ & - & - & - & - & - \\
\hline
\end{tabular}

\footnotetext{
${ }^{1}$ Includes landslides, avalanches, and debris flows. Significant potential for displacements and impacts.

${ }^{2}$ Where fine grained (sand and silt), saturated, and thawed, there is significant potential for loss of shear strength during conditions of dynamic loading (shaking).

${ }^{3}$ Where perennially frozen, contains significant pervasive or massive ice, or both. During and after melting, susceptible to excessive and differential settlements and loss of shear strength.

${ }^{4}$ Includes seasonal stream icings.

${ }^{5}$ Includes slushflows during periods of rapid spring melting in Brooks Range and on Seward Peninsula.

${ }^{6}$ Steep bedrock outcrops are susceptible to snow avalanching, However, this hazard is related to the topography, which was identified during this study by a study of topographic maps. Landslides in bedrock are mapped as slope failures, not bedrock.
} 
Table 3. (cont'd.)

\begin{tabular}{|l|c|c|c|c|}
\hline \multicolumn{1}{|c|}{$\begin{array}{c}\text { MATERIALS } \\
\text { CLASS }\end{array}$} & $\begin{array}{c}\text { Rapid mass } \\
\text { movements }\end{array}$ & $\begin{array}{c}\text { Significant } \\
\text { liquefaction } \\
\text { potential }\end{array}$ & $\begin{array}{c}\text { Significant ground- } \\
\text { ice content }\end{array}$ & Seasonal flooding \\
\hline $\begin{array}{l}\text { Snow } \\
\text { avalanching }\end{array}$ \\
\hline $\begin{array}{l}\text { All other lithologies (BO) } \\
\text { Undifferentiated, unknown } \\
\text { bedrock (BU) }\end{array}$ & - & - & - & - \\
\hline Glacial ice (ICE) & - & - & - & - \\
\hline
\end{tabular}


Table 4. Zones of permafrost identified during corridor evaluation

\begin{tabular}{|c|l|}
\hline Zone & \multicolumn{1}{|c|}{ Explanation } \\
\hline Continuous & $\begin{array}{l}\text { Perennially frozen ground present beneath > 90 percent of } \\
\text { landscape. (Ferrians, 1965; Kreig and Reger, 1982) }\end{array}$ \\
\hline $\begin{array}{c}\text { Transitional from } \\
\text { Continuous to } \\
\text { Discontinuous }\end{array}$ & $\begin{array}{l}\text { Both continuous and discontinuous permafrost are present. } \\
\text { (Ferrians, 1965) }\end{array}$ \\
\hline $\begin{array}{c}\text { Discontinuous } \\
\text { of lanndscape (Kreig and Reger, 1982). Generally absent } \\
\text { close to large water bodies. (Ferrians, 1965) }\end{array}$ \\
\hline $\begin{array}{c}\text { Transitional from } \\
\text { Discontinuous to } \\
\text { Sporadic }\end{array}$ & $\begin{array}{l}\text { Both discontinuous and sporadic permafrost are present. } \\
\text { (Ferrians, 1965) }\end{array}$ \\
\hline Sporadic & $\begin{array}{l}\text { Perennially frozen ground present beneath }<\text { 50 percent of } \\
\text { landscape; generally present as isolated, relict masses at } \\
\text { considerable depth or as local, thin lenses near ground } \\
\text { surface where peat is thick enough to provide adequate } \\
\text { insulation. (Kreig and Reger, 1982) }\end{array}$ \\
\hline No permafrost & $\begin{array}{l}\text { Perennially frozen ground is not present even in favorable } \\
\text { locations. (Ferrians, 1965) }\end{array}$ \\
\hline
\end{tabular}


Table 5. Distribution of permafrost zones by quadrangle crossed by proposed 10-mile-wide corridors in Alaska (after Ferrians, 1965)

\begin{tabular}{|c|c|c|}
\hline $\begin{array}{l}\text { Permafrost } \\
\text { Zone }\end{array}$ & $\begin{array}{l}\text { Quadrangles } \\
\text { Included }\end{array}$ & $\begin{array}{l}\text { Percent of all } \\
\text { Quadrangles }\end{array}$ \\
\hline Continuous & $\begin{array}{l}\text { Barrow, Barter Island, Beechey Pont, } \\
\text { Chandler Lake, DeLong Mountains, } \\
\text { Demarcation Point, Flaxman Island, Harrison } \\
\text { Bay, Howard Pass, Ikpikpuk River, Killik } \\
\text { River, Lookout Ridge, Meade River, } \\
\text { Misheguk Mountain, Mount Michelson, } \\
\text { Philip Smith Mountains, Sagavanirktok, } \\
\text { Teshekpuk, Umiat, Utukok River (n=20) }\end{array}$ & 25.6 \\
\hline $\begin{array}{l}\text { Transitional from } \\
\text { Continuous to } \\
\text { Discontinuous }\end{array}$ & $\begin{array}{l}\text { Ambler River, Baird Mountains, Bettles, } \\
\text { Chandalar, Hughes, Noatak, Selawik, } \\
\text { Shungnak, Survey Pass, Wiseman }(n=10)\end{array}$ & 12.8 \\
\hline Discontinuous & $\begin{array}{l}\text { Bendeleben, Black River, Candle, Charley } \\
\text { River, Circle, Healy, Holy Cross, Kateel } \\
\text { River, Livengood, Melozitna, Norton Bay, } \\
\text { Nulato, Ruby, Solomon, Tanana, Unalakleet } \\
(\mathrm{n}=15)\end{array}$ & 19.2 \\
\hline Sporadic & Dillingham, Iliamna, Naknek $(\mathrm{n}=3)$ & 3.8 \\
\hline \multirow[t]{2}{*}{ No permafrost } & $\begin{array}{l}\text { Bradfield Canal, Chignik, Cold Bay, Juneau, } \\
\text { Karluk, Mount Katmai, Petersburg, Port } \\
\text { Moller, Taku River, Ugashik, Yakutat }(\mathrm{n}=11)\end{array}$ & 14.1 \\
\hline & Total: & 99.9 \\
\hline
\end{tabular}


Table 6. Distribution of stream drainages subject to periodic outburst floods in 10-mile-wide corridors containing proposed access routes (Post and Mayo, 1971)

\begin{tabular}{|c|c|}
\hline Quadrangle & $\begin{array}{l}\text { Drainage affected by } \\
\text { outburst flooding }\end{array}$ \\
\hline Bering Glacier & $\begin{array}{l}\text { Campbell River, Edwardes River, Kiklukh River, Nichawak } \\
\text { River, Seal River, Tashalich River, Tsirat River, Tsiu River }\end{array}$ \\
\hline Bradfield Canal & Stikine River \\
\hline Cordova & $\begin{array}{l}\text { Bering River, Campbell River, Copper River, Edwardes River, } \\
\text { Gadil River, Martin River, Nichawak River, Oaklee River, } \\
\text { Sheep Creek }\end{array}$ \\
\hline Healy & Nenana River \\
\hline Juneau & Stream draining North Fork Norris Glacier \\
\hline Lime Hills & South Fork Kuskokwim River, Swift River \\
\hline Mount McKinley & Foraker River, Herron River, McKinley River \\
\hline Taku River & Taku River \\
\hline Tyonek & $\begin{array}{l}\text { Beluga River, Hayes River, Skwentna River, Susitna River, } \\
\text { Yentna River }\end{array}$ \\
\hline
\end{tabular}


Table 7. Distribution of stream drainages subject to seasonal icings (naleds) in proposed 10mile-wide corridors containing proposed access routes (Dean, 1984a). Numerous small, unnamed streams are not listed.

\begin{tabular}{|c|c|}
\hline Quadrangle & Stream affected by seasonal icings[dsp1] \\
\hline Ambler River & $\begin{array}{l}\text { Agnes Creek, Akiak Creek, Akillik River, Ambler River, Cross Creek, Cutler } \\
\text { River, Hunt River, Ipnelivik River, Kogoluktuk River, Miluet Creek, } \\
\text { Naniratkohort Creek, Nekakte Creek, Noatak River, Nushralutak Creek, Redstone } \\
\text { River, Tunukuchiak Creek }\end{array}$ \\
\hline Baird Mountains & Squirrel River, Tutuksuk River \\
\hline Barter Island & Okpilak River \\
\hline Bendeleben & $\begin{array}{l}\text { Boston Creek, Fish River, Lava Creek, Nutmoyuk Creek, Ophir Creek, Telephone } \\
\text { Creek, Virginia Creek, Windy Creek }\end{array}$ \\
\hline Bethel & Aniak River, Kipchuk River \\
\hline Bettles & West Fork Henshaw (Sozhekla) Creek \\
\hline Black River & Grayling Fork Black River, Salmon Fork Black River \\
\hline Candle & $\begin{array}{l}\text { Brush Creek, Connolly Creek, Little Kalusuk Creek, North Fork Buckland River, } \\
\text { West Fork Buckland River }\end{array}$ \\
\hline Chandalar & Crooked Creek, Slate Creek, South Fork Koyukuk River \\
\hline Chandler Lake & $\begin{array}{l}\text { Anaktuvuk River, Chandler River, Cobblestone Creek, Confusion Creek, } \\
\text { Desolation Creek, Kanayut River, May Creek, Nanushuk River, Siksikpuk River }\end{array}$ \\
\hline Charley River & Charley River, Webber Creek \\
\hline Chignik & Barbara Creek, Birthday Creek, Plenty Bear Creek, Reindeer Creek \\
\hline Cold Bay & Joshua Green River \\
\hline Cordova & Copper River \\
\hline De Long Mountains & Wulik River \\
\hline Demarcation Point & Okpilak River \\
\hline Dillingham & $\begin{array}{l}\text { Alagnak River, Kokwok River, Kvichak River, Stuyahok River, unnamed } \\
\text { tributaries to Kaskanak Creek, unnamed tributaries to Mulchatna River }\end{array}$ \\
\hline Flaxman Island & Hulahula River \\
\hline Healy & Nenana River, Savage River, Sushana River, Teklanika River \\
\hline Holy Cross & Beaver Creek \\
\hline Howard Pass & Colville River, Nigu River, Rough Mountain Creek \\
\hline Hughes & Unnamed stream \\
\hline Iditarod & Unnamed north tributary to Bonanza Creek \\
\hline Iliamna & Dunuletak Creek, Iliamna River, Koktuli River, Paint River \\
\hline
\end{tabular}


Table 7. (cont'd).

\begin{tabular}{|c|c|}
\hline Quadrangle & Stream affected by seasonal icings[dsp1] \\
\hline Kantishna River & Unnamed tributaries to Cosna River, unnamed tributaries to Titna River \\
\hline Karluk & Unnamed tributary to Kejulik River \\
\hline Kateel River & Kateel River \\
\hline Killik River & $\begin{array}{l}\text { Killik River, Kurupa River, Okpikruak River, Oolamnagavik River, Travelair } \\
\text { Creek }\end{array}$ \\
\hline Lime Hills & $\begin{array}{l}\text { Big River, Hartman River, North Fork Big River, Ptarmigan Creek, South Fork } \\
\text { Kuskokwim River, Stony River, Styx River }\end{array}$ \\
\hline Livengood & Hutlinana Creek \\
\hline McGrath & $\begin{array}{l}\text { Big River, Jones River, South Fork Kuskokwim River, Tatina River, Windy Fork } \\
\text { Kuskokwim River }\end{array}$ \\
\hline Medfra & $\begin{array}{l}\text { Black Creek, Brown's Fork Susulatna River, Dennis Creek, East Fork } \\
\text { Kuskokwim River, Eden Creek, Granite Creek, Hardscrabble Creek, North Fork } \\
\text { Jones Creek, Shepherd Creek, Silver Creek, South Fork Sulatna River, Timber } \\
\text { Creek, Tonzona River, unnamed tributaries to Susulatna River. }\end{array}$ \\
\hline Mount McKinley & $\begin{array}{l}\text { Bearpaw River, Birch Creek, Clearwater Fork Toklat River, East Fort Toklat } \\
\text { River, Fish Creek, Foraker River, Herron River, McKinley Creek, Moonlight } \\
\text { Creek, Shisnona River, Slippery Creek, Stony Creek, Toklat River }\end{array}$ \\
\hline Mount Michelson & $\begin{array}{l}\text { Canning River, Hulahula River, Itkilyariak Creek, Katakturuk River, March } \\
\text { Creek, Sadlerochit River, Tamayariak River }\end{array}$ \\
\hline Naknek & King Salmon River, Naknek River \\
\hline Noatak & Agashashok River, Evaingiknuk Creek, Kiyak Creek, Noatak River \\
\hline Norton Bay & $\begin{array}{l}\text { Anakeksik Creek, Egavik Creek, North River, Nulato River, Shaktoolik River, } \\
\text { Unalakleet River, Ungalik River }\end{array}$ \\
\hline Nulato & $\begin{array}{l}\text { Arvesta Creek, Gisasa River, Little Mud River, Nulato River, West Fork Little } \\
\text { Mud River }\end{array}$ \\
\hline Philip Smith Mountains & Unnamed tributary to Itkillik River \\
\hline Port Moller & Unnamed streams \\
\hline Ruby & Unnamed tributaries to Sulukna River, unnamed tributaries to Telsitna River \\
\hline Russian Mission & $\begin{array}{l}\text { Aniak River, Buckstock River, Kipchuk River, Mission Creek, Salmon River, } \\
\text { Timber Creek }\end{array}$ \\
\hline Sagavanirktok & Kavik River, Sagavanirktok River, Shaviovik River, Toolik River \\
\hline Selawik & Unnamed tributary to Tagagawik River \\
\hline Shungnak & Black River, Cosmos Creek, Ingruksukruk Creek, Wesley Creek \\
\hline Sleetmute & Buckstock River, Timber Creek \\
\hline Solomon & Eagle Creek, Kachauik River, Niukluk River, Richter Creek, Yuonglik River \\
\hline Survey Pass & Malamute Fork Alatna River \\
\hline Tanana & $\begin{array}{l}\text { Big Denver Creek, Dagislakhna Creek, Hutlinana Creek, Nethkahati Creek, } \\
\text { Slokhenjikh Creek, Tenmile Creek }\end{array}$ \\
\hline Teshekpuk & Ikpikpuk River \\
\hline
\end{tabular}


Table 7. (cont'd).

\begin{tabular}{|c|l|}
\hline $\begin{array}{c}\text { Quadrangle } \\
\text { Tyonek }\end{array}$ & \multicolumn{1}{|c|}{ Stream affected by seasonal icings[dsp1] } \\
\hline Umiat & Anaktuvuk River, Itkillik River, Kuparuk River, Nanushuk River \\
\hline Unalakleet & North River, Swift River, unnamed tributaries of Unalakleet River \\
\hline Utukok River & Utukok River \\
\hline Wiseman & Middle Fork Koyukuk River \\
\hline
\end{tabular}


Table 8. Criteria of classes of fault activity used in geologic evaluation of proposed access corridors in Alaska (modified from Howard and others, 1978)

\begin{tabular}{|c|l|}
\hline \multicolumn{1}{|c|}{ Class } & \multicolumn{1}{c|}{ Criteria } \\
\hline Definitely active & $\begin{array}{l}\text { Movement demonstrated during last 10,000 } \\
\text { years }\end{array}$ \\
\hline Probably active & $\begin{array}{l}\text { Possible evidence of displacement during last } \\
10,000 \text { years }\end{array}$ \\
\hline Possibly active & $\begin{array}{l}\text { Possible evidence of displacement during last } \\
500,000 \text { years }\end{array}$ \\
\hline
\end{tabular}


Table 9. Criteria for data-quality classes used during geologic evaluation of proposed access corridors in Alaska

\begin{tabular}{|c|l|}
\hline Class & \multicolumn{1}{|c|}{ Criteria } \\
\hline Very good & $\begin{array}{l}\text { One or more 1:63,360- or larger scale geologic } \\
\text { maps available; verified by numerous detailed field } \\
\text { observations, multiple laboratory tests, or both; } \\
\text { interpretation of 1:65,000- or larger scale aerial } \\
\text { photographs has considerable field verification. }\end{array}$ \\
\hline Good & $\begin{array}{l}\text { A 1:63,360- or larger scale geologic map available; } \\
\text { partially verified by field studies, a few laboratory } \\
\text { tests, or both; interpretation of 1:65,000- or larger } \\
\text { scale aerial photographs has limited or spotty field } \\
\text { verification. }\end{array}$ \\
\hline Moderate & $\begin{array}{l}\text { A 1:250,000- or 1:125,000-scale geologic map } \\
\text { available; known to be generally reliable because } \\
\text { of limited field observations, a few laboratory tests, } \\
\text { or both; interpretation of 1:65,000- or larger scale } \\
\text { aerial photographs is not verified by field } \\
\text { observations. }\end{array}$ \\
\hline Fair & $\begin{array}{l}\text { A reconnaissance-series 1:250,000-scale geologic } \\
\text { map available but has not been reliably verified by } \\
\text { field observations, laboratory tests, or both; } \\
\text { interpretation of 1:250,000-scale satellite imagery } \\
\text { or topographic maps are composite and } \\
\text { identification of individual landforms is not } \\
\text { possible. }\end{array}$ \\
\hline Poor & $\begin{array}{l}\text { Only 1:1,000,000-scale geologic map available; } \\
\text { only some obvious composite landforms can be } \\
\text { identified on 1:63,360-scale or larger topographic } \\
\text { maps. }\end{array}$ \\
\hline
\end{tabular}




\section{APPENDIX 1}

IDENTIFICATION OF 1:60,000-SCALE FALSE-COLOR INFRARED AERIAL PHOTOGRAPHS OF ALK 60 CIR SERIES INTERPRETED TO PREPARE GEOLOGIC, MATERIALS, AND HAZARDS MAPS DURING THIS INVESTIGATION

\begin{tabular}{|c|c|c|c|}
\hline $\begin{array}{c}\text { PRIMARY } \\
\text { QUADRANGLE }{ }^{1}\end{array}$ & $\begin{array}{c}\text { DATE OF } \\
\text { PHOTOGRAPHY }\end{array}$ & $\begin{array}{c}\text { ACCESSION OR } \\
\text { ROLL } \\
\text { NUMBER } \\
\end{array}$ & $\begin{array}{c}\text { FRAME } \\
\text { NUMBERS }\end{array}$ \\
\hline Baird Mountains & July 5, 1978 & Roll 15 & $69-77$ \\
\hline " & " & $"$ & $83-94$ \\
\hline " & " & " & $107-125$ \\
\hline " & July 14,1978 & Roll 29 & $225-232$ \\
\hline$"$ & July 14,1979 & 2788 & $2888-2891$ \\
\hline$"$ & $"$ & $"$ & $2903-2910$ \\
\hline " & $"$ & $"$ & $2927-2931$ \\
\hline " & July 5,1978 & 2626 & $6075-6085$ \\
\hline Barter Island & July 13,1979 & 2786 & $2391-2394$ \\
\hline " & $"$ & $"$ & $2515-2521$ \\
\hline $\begin{array}{l}\text { Bettles (Hughes, } \\
\text { Melozitna) }\end{array}$ & June 27, 1978 & Roll 9 & $303-308$ \\
\hline (Hughes) & " & " & $370-376$ \\
\hline (Hughes) & July 25,1981 & 2998 & $1641-1658$ \\
\hline (Hughes) & $"$ & " & $1670-1680$ \\
\hline (Hughes) & " & $"$ & $1777-1783$ \\
\hline (Hughes) & August 2, 1981 & 3004 & $1905-1911$ \\
\hline Bettles & " & $"$ & $2000-2006$ \\
\hline (Hughes) & July 14,1979 & 2788 & $2552-2557$ \\
\hline (Hughes) & " & $"$ & $2659-2664$ \\
\hline (Hughes) & August 1, 1979 & 2794 & $3549-3556$ \\
\hline (Hughes) & " & " & $3562-3572$ \\
\hline Bettles & " & $"$ & $3620-3633$ \\
\hline$"$ & $"$ & $"$ & $3646-3653$ \\
\hline Bradfield Canal & August 11, 1979 & 2804 & $4365-4368$ \\
\hline$"$ & August 12,1979 & 2806 & $4640-4647$ \\
\hline$"$ & " & $"$ & $4703-4716$ \\
\hline$"$ & " & $"$ & $4726-4730$ \\
\hline$"$ & " & $"$ & $4784-4790$ \\
\hline$"$ & $"$ & $"$ & $4847-4851$ \\
\hline " & August 14,1979 & 2811 & $5054-5057$ \\
\hline
\end{tabular}

\footnotetext{
${ }^{1}$ Parts of corridors in quadrangles inside parentheses are also covered by photographs listed, but represent less land area than primary quadrangle.
} 


\begin{tabular}{|c|c|c|c|}
\hline $\begin{array}{c}\text { PRIMARY } \\
\text { QUADRANGLE }\end{array}$ & $\begin{array}{c}\text { DATE OF } \\
\text { PHOTOGRAPHY }\end{array}$ & $\begin{array}{c}\text { ACCESSION OR } \\
\text { ROLL } \\
\text { NUMBER } \\
\end{array}$ & $\begin{array}{c}\text { FRAME } \\
\text { NUMBERS }\end{array}$ \\
\hline Dillingham & August 15,1984 & 3391 & $76-82$ \\
\hline " & " & " & $150-156$ \\
\hline$"$ & August 41982 & 3110 & $180-184$ \\
\hline$"$ & $"$ & " & $198-204$ \\
\hline$"$ & " & $"$ & $245-249$ \\
\hline$"$ & $"$ & " & $283-287$ \\
\hline " & August 20, 1982 & 3122 & $1775-1780$ \\
\hline " & August 19,1983 & 3271 & $5604-5610$ \\
\hline$"$ & $"$ & $"$ & $5614-5623$ \\
\hline$"$ & $"$ & $"$ & $5665-5669$ \\
\hline " & $"$ & $"$ & $5677-5682$ \\
\hline$"$ & July 17,1980 & 2907 & $5794-5798$ \\
\hline " & " & $"$ & $5808-5813$ \\
\hline$"$ & $"$ & " & $5832-5836$ \\
\hline " & August 8, 1984 & 3376 & $6622-6627$ \\
\hline$"$ & & $"$ & $6661-6668$ \\
\hline Holy Cross & July 17,1980 & Roll 17 & $42-46$ \\
\hline$"$ & July 21,1980 & Roll 14 & $60-77$ \\
\hline " & July 17,1980 & Roll 7 & $95-101$ \\
\hline " & July 21,1980 & Roll 14 & $118-136$ \\
\hline " & July 17,1980 & 2907 & $5620-5623$ \\
\hline " & July 19,1980 & 2911 & $6257-6268$ \\
\hline " & July 23,1980 & 2917 & $7173-7177$ \\
\hline$"$ & & 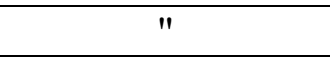 & $7220-7239$ \\
\hline$"$ & July 24,1980 & 2919 & $7514-7529$ \\
\hline$"$ & (u & $"$ & $7571-7583$ \\
\hline & & & 2 \\
\hline Hughes & July 12,1979 & 2784 & $1672-1684$ \\
\hline " & August 2, 1981 & 3004 & $1970-1991$ \\
\hline Iditarod & July 21,1980 & Roll 14 & $40-49$ \\
\hline " & 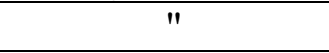 & $"$ & $137-142$ \\
\hline$"$ & " & $"$ & $147-154$ \\
\hline$"$ & July 17,1980 & Roll 7 & $157-166$ \\
\hline " & July 21,1980 & Roll 14 & $221-227$ \\
\hline (Holy Cross) & " & 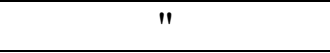 & $194-211$ \\
\hline Iditarod & $"$ & $"$ & $296-301$ \\
\hline$"$ & August 21,1982 & 3124 & $2091-2098$ \\
\hline (Sleetmute) & July 19,1980 & 2911 & $6246-6254$ \\
\hline Juneau & August 11, 1979 & 2804 & $4257-4260$ \\
\hline
\end{tabular}




\begin{tabular}{|c|c|c|c|}
\hline $\begin{array}{c}\text { PRIMARY } \\
\text { QUADRANGLE }^{1}\end{array}$ & $\begin{array}{c}\text { DATE OF } \\
\text { PHOTOGRAPHY }\end{array}$ & $\begin{array}{c}\text { ACCESSION OR } \\
\text { ROLL } \\
\text { NUMBER } \\
\end{array}$ & $\begin{array}{c}\text { FRAME } \\
\text { NUMBERS }\end{array}$ \\
\hline$"$ & $"$ & 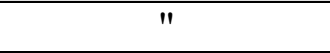 & $4322-4325$ \\
\hline$"$ & $"$ & Roll 11 & $384-387$ \\
\hline Lake Clark & August 3,1982 & 3109 & $13-16$ \\
\hline " & August 13,1984 & 3385 & $100-104$ \\
\hline$"$ & June 20,1978 & 2616 & $4704-4710$ \\
\hline$"$ & " & " & $4753-4756$ \\
\hline$"$ & " & " & $4852-4857$ \\
\hline$"$ & August 26,1978 & 2667 & $7569-7572$ \\
\hline$"$ & " & " & $7741-7745$ \\
\hline$"$ & $"$ & $"$ & $7795-7799$ \\
\hline Lime Hills & August 13,1984 & 3385 & $79-92$ \\
\hline " & " & $"$ & $125-129$ \\
\hline$"$ & " & $"$ & $134-137$ \\
\hline$"$ & July 21,1980 & Roll 14 & $345-349$ \\
\hline$"$ & July 20,1980 & 2913 & $6611-6626$ \\
\hline (Lake Clark) & August 25,1978 & 2664 & $7353-7358$ \\
\hline Lime Hills & August 26,1978 & 2667 & $7806-7813$ \\
\hline " & " & " & $7828-7832$ \\
\hline$"$ & " & $"$ & $7835-7841$ \\
\hline$"$ & " & $"$ & $7842-7846$ \\
\hline$"$ & $"$ & $"$ & $7850-7855$ \\
\hline Melozitna & June 27,1978 & Roll 9 & $183-191$ \\
\hline " & " & $"$ & $208-213$ \\
\hline$"$ & $"$ & " & $281-287$ \\
\hline Naknek & August 15,1984 & 3391 & $287-292$ \\
\hline$"$ & $"$ & $"$ & $315-320$ \\
\hline$"$ & " & $"$ & $428-432$ \\
\hline$"$ & " & $"$ & $441-446$ \\
\hline (Dillingham) & August 20,1982 & 3122 & $1775-1780$ \\
\hline Naknek & $"$ & $"$ & $1796-1800$ \\
\hline 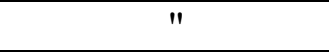 & $"$ & $"$ & $1888-1894$ \\
\hline$"$ & August 27, 1983 & 3281 & $6667-6671$ \\
\hline$"$ & 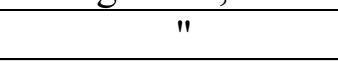 & $"$ & $6715-6721$ \\
\hline Noatak & July 14,1979 & 2788 & $2772-2777$ \\
\hline$"$ & 1 & " & $2793-2797$ \\
\hline$"$ & $"$ & $"$ & $2864-2868$ \\
\hline$"$ & $"$ & $"$ & $2878-2888$ \\
\hline$"$ & $"$ & $"$ & $2931-2941$ \\
\hline
\end{tabular}




\begin{tabular}{|c|c|c|c|}
\hline $\begin{array}{c}\text { PRIMARY } \\
\text { QUADRANGLE }^{1}\end{array}$ & $\begin{array}{c}\text { DATE OF } \\
\text { PHOTOGRAPHY }\end{array}$ & $\begin{array}{c}\text { ACCESSION OR } \\
\text { ROLL } \\
\text { NUMBER } \\
\end{array}$ & $\begin{array}{c}\text { FRAME } \\
\text { NUMBERS }\end{array}$ \\
\hline$"$ & $"$ & " & $2946-2951$ \\
\hline$"$ & July 3,1978 & 2624 & $5868-5872$ \\
\hline Ruby & July 23,1980 & Roll 15 & $23-28$ \\
\hline " & " & $"$ & $42-49$ \\
\hline$"$ & " & " & $136-141$ \\
\hline$"$ & " & $"$ & $153-159$ \\
\hline$"$ & " & $"$ & $190-196$ \\
\hline$"$ & $"$ & $"$ & $209-216$ \\
\hline$"$ & " & $"$ & $326-331$ \\
\hline Sagavanirktok & June 28,1978 & 2622 & $5604-5613$ \\
\hline$"$ & " & $"$ & $5632-5640$ \\
\hline " & July 13,1979 & 2786 & $2359-2369$ \\
\hline$"$ & " & " & $2417-2429$ \\
\hline$"$ & July 8,1978 & Roll 19 & $294-303$ \\
\hline$"$ & " & $"$ & $307-316$ \\
\hline Sleetmute & August 13,1984 & 3385 & $70-78$ \\
\hline Sleetmute & August 5, 1982 & 3112 & $313-326$ \\
\hline Sleetmute & $"$ & $"$ & $355-357$ \\
\hline " & " & $"$ & $370-376$ \\
\hline " & " & $"$ & $379-388$ \\
\hline " & " & $"$ & $393-401$ \\
\hline$"$ & June 20,1978 & 2616 & $4647-4649$ \\
\hline$"$ & July 11,1980 & 2911 & $6285-6290$ \\
\hline$"$ & July 20,1980 & 2913 & $6689-6690$ \\
\hline Taku River & August 11,1979 & 2804 & $4403-4407$ \\
\hline$"$ & August 12,1979 & 2806 & $4890-4896$ \\
\hline Taylor Mountains & July 20,1980 & Roll 10 & $13-17$ \\
\hline$"$ & August 13,1984 & 3385 & $14-18$ \\
\hline$"$ & $"$ & $"$ & $50-54$ \\
\hline (Dillingham) & August 15,1984 & 3391 & $87-97$ \\
\hline Taylor Mountains & July 20,1980 & Roll 10 & $222-224$ \\
\hline " & 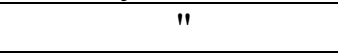 & " & $250-254$ \\
\hline (Sleetmute) & August 5, 1982 & 3112 & $327-349$ \\
\hline Taylor Mountains & June 20,1978 & 2616 & $4868-4869$ \\
\hline$"$ & August 18,1983 & 3269 & $4870-4875$ \\
\hline$"$ & " & $"$ & $4917-4915$ \\
\hline$"$ & $"$ & $"$ & $4919-4925$ \\
\hline$"$ & " & $"$ & $4944-4948$ \\
\hline
\end{tabular}




\begin{abstract}
APPENDIX 2
LISTING BY QUADRANGLE OF TECHNICAL REFERENCES USED DURING EVALUATION OF GEOLOGY, CONSTRUCTION MATERIALS, AND NATURAL HAZARDS IN PROPOSED ACCESS CORRIDORS IN ALASKA
\end{abstract}

Ambler River

Dean, K.G., 1984a, Stream-icing zones in Alaska: Alaska Division of Geological \& Geophysical Surveys Report of Investigations 84-16, 20 p., scale 1:250,000, 102 sheets.

Hamilton, T.D., 1984, Surficial geologic map of the Ambler River Quadrangle, Alaska: U.S. Geological Survey Miscellaneous Field Studies Map MF-1678, scale 1:250,000, 1 sheet.

Pessel, G.H., and Brosgé, W.P., 1977, Preliminary reconnaissance geologic map of Ambler River Quadrangle, Alaska: U.S. Geological Survey Open-file Report 77-28, scale 1:250,000, 1 sheet.

\title{
Anchorage
}

Bruhn, R.L., 1979, Holocene displacements measured by trenching the Castle Mountain fault near Houston, Alaska, in Short Notes on Alaskan Geology: Alaska Division of Geological \& Geophysical Surveys., Geologic Report 61, p. 1-4.

Burns, L.E., Pessel, G.H., Little, T.A., Pavlis, T.L., Newberry, R.J., Winkler, G.R., and Decker, John, 1991, Geology of the northern Chugach Mountains, southcentral Alaska: Alaska Division of Geological \& Geophysical Surveys Professional Report 94, 63 p., scale $1: 63,360,2$ sheets.

Detterman, R.L., Plafker, George, Hudson, Travis, Tysdal, R.G., and Pavoni, Nazario, 1974, Surface geology and Holocene breaks along the Susitna segment of the Castle Mountain fault, Alaska: U.S. Geological Survey Miscellaneous Field Studies Map MF-618, scale $1: 24,000,1$ sheet.

Detterman, R.L., Plafker, George, Hudson, Travis, and Tysdal, R.G., 1975, Earthquake hazards investigated along Castle Mountain fault in Matanuska Valley, in Yount, M.E., ed., United States Geological Survey Alaska Program, 1975: U.S. Geological Survey Circular 722, p. 48.

Detterman, R.L., Plafker, George, Tysdal, R.G., and Hudson, Travis, 1976, Geology and surface features along part of the Talkeetna segment of the Castle Mountain-Caribou fault system, Alaska: U.S. Geological Survey Miscellaneous Field Studies MF-738, scale 1:63,360, 1 sheet. 
Grantz, Arthur, 1961, Geologic map and cross sections of the Anchorage D-2 Quadrangle and northeasternmost part of the Anchorage D-3 Quadrangle, Alaska: U.S. Geological Survey Miscellaneous Geologic Investigations Map I-342, scale 1:48,000, 1 sheet.

Lahr, J.C., and Page, R.A., 1986, Evidence for activity of the Castle Mountain fault system: A review for the 1985 NEPEC Workshop, in Shearer, C.F., ed., Minutes of the National Earthquake Prediction Evaluation Council, September 8 and 9, 1985, Anchorage, Alaska: U.S. Geological Survey Open-file Report 86-92, Appendix A6, p. 103-118.

Lahr, J.C., Page, R.A., Fogleman, K.A., and Stephens, C.D., 1985, New evidence for activity on the Talkeetna segment, Castle Mountain-Caribou fault system: The 1984 Sutton earthquake, in Bartsch-Winkler, Susan, ed., The United States Geological Survey in Alaska: Accomplishments during 1984: U.S. Geological Survey Circular 967, p. 62-63.

Lahr, J.C., Page, R.A., Stephens, C.D., and Fogleman, K.A., 1986, Sutton, Alaska, earthquake of 1984: Evidence for activity on the Talkeetna segment of the Castle Mountain fault system: Bulletin of the Seismological Society of America, v. 76, no. 4, p. 967-983.

Magoon, L.B., Adkison, W.L., and Egbert, R.M., 1976, Map showing geology, wildcat wells, Tertiary plant fossil localities, K-Ar age dates, and petroleum operations, Cook Inlet area, Alaska: U.S. Geological Survey Miscellaneous Investigations Map I-1019, scale 1:250,000, 3 sheets.

Page, Robert, and Lahr, John, 1971, Measurements for fault slip on the Denali, Fairweather, and Castle Mountain faults, Alaska: Journal of Geophysical Research, v.76, no. 35, p. 85348543.

Reger, R.D., 1978, Reconnaissance geology of the new capital site and vicinity, Anchorage Quadrangle, Alaska: Alaska Division of Geological \& Geophysical Surveys Open-file Report 113A, scale 1:63,360, 1 sheet.

1981a, Geologic and materials maps of the Anchorage C-8 SW Quadrangle, Alaska: Alaska Division of Geological \& Geophysical Surveys Geologic Report 68, scale 1:25,000, 2 sheets.

1981b, Geologic and materials maps of the Anchorage B-8 NW Quadrangle, Alaska: Alaska Division of Geological \& Geophysical Surveys Geologic Report 70, scale 1:25,000, 2 sheets.

1981c, Geologic and materials maps of the Anchorage C-8 NE Quadrangle, Alaska: Alaska Division of Geological \& Geophysical Surveys Geologic Report 65, scale 1:25,000, 2 sheets.

Reger, R.D., and Updike, R.G., 1983, Upper Cook Inlet and the Matanuska Valley, in Péwé, T.L., and Reger, R.D., eds., Guidebook to permafrost and Quaternary geology along the 
Richardson and Glenn Highways between Fairbanks and Anchorage, Alaska: Alaska Division of Geological \& Geophysical Surveys Guidebook 1, p. 185-263, scale 1:250,000, 1 sheet.

\section{Baird Mountains}

Dean, K.G., 1984a, Stream-icing zones in Alaska: Alaska Division of Geological \& Geophysical Surveys Report of Investigations 84-16, 20 p., scale 1:250,00O, 102 sheets.

Karl, S.M., Dumoulin, J.A., Ellersieck, Inyo, Harris, A.G., and Schmidt, J.M., 1990, Preliminary geologic map of the Baird Mountains and part of the Selawik Quadrangles, Alaska: U.S. Geological Survey Open-file Report 89-551, 65 p., scale 1:250,000, 1 sheet.

Patton, W.W., Jr., and Miller, T.P., 1968, Regional geologic map of the Selawik and southeastern Baird Mountains Quadrangles, Alaska: U.S. Geological Survey Miscellaneous Geologic Investigations Map I-530, scale 1:250,000, 1 sheet.

\section{Barrow}

Williams, J.R., and Carter, L.D., 1984, Engineering-geologic maps of northern Alaska, Barrow Quadrangle: U.S. Geological Survey Open-file Report 84-124, 39 p., scale 1:250,000, 2 sheets.

Barter Island

Dean, K.G., 1984a, Stream-icing zones in Alaska: Alaska Division of Geological \& Geophysical Surveys Report of Investigations 84-16, 20 p., scale 1:250,000, 102 sheets.

Beechey Point

Hickmott, D.R., 1986, Surficial-geologic map of the Beechey Point A-2 Quadrangle, Alaska: Alaska Division of Geological \& Geophysical Surveys Report of Investigations 86-7, scale $1: 63,360,1$ sheet.

Rawlinson, S.E., 1986a, Surficial-geologic map of the Beechey Point A-3 Quadrangle, Alaska: Alaska Division of Geological \& Geophysical Surveys Report of Investigations 86-8, scale $1: 63,360,1$ sheet.

1986b, Surficial-geologic map of the Beechey Point A-4 Quadrangle, Alaska: Alaska Division of Geological \& Geophysical Surveys Report of Investigations 86-9, scale 1:63,360, 1 sheet.

1990, Surficial geology and morphology of the Alaskan central Arctic Coastal Plain: Fairbanks, University of Alaska PhD dissertation, 311 p., scale 1:63,360, 6 sheets. 
Bendeleben

Dean, K.G., 1984a, Stream-icing zones in Alaska: Alaska Division of Geological \& Geophysical Surveys Report of Investigations 84-16, 20 p., scale 1:250,000, 102 sheets.

Hudson, Travis, and Plafker, George, 1978, Kigluaik and Bendeleben faults, Seward Peninsula, in Johnson, K.M., ed., The United States Geological Survey in Alaska: Accomplishments during 1977: U.S. Geological Survey Circular 772-B, p. B47-B50.

Kaufman, D.S., 1985, Windy Creek and Crater Creek faults, Seward Peninsula, in BartschWinkler, Susan, and Reed, K.M., eds., The United States Geological Survey in Alaska: Accomplishments during 1983: U.S. Geological Survey Circular 945, p. 22-24.

1986, Surficial geologic map of the Solomon, Bendeleben, and southern part of the Kotzebue Quadrangle, western Alaska: U.S. Geological Survey Miscellaneous Field Studies Map MF-1838A, scale 1:250,000, 1 sheet.

Till, A.B., Dumoulin, J.A., Gamble, B.M., Kaufman, D.S., and Carroll, P.I., 1986, Preliminary geologic map and fossil data, Solomon, Bendeleben, and southern Kotzebue Quadrangles, Seward Peninsula, Alaska: U.S. Geological Survey Open-file Report 86-276, 60 p., scale $1: 250,000,3$ sheets.

\section{Bering Glacier}

Miller, D.J., 1961, Geology of the Katalla district, Gulf of Alaska Tertiary province, Alaska: U.S. Geological Survey Open-file Report 61-99, scale 1:96,000, 2 sheets.

1971, Geologic map of the Yakataga district, Gulf of Alaska Tertiary province, Alaska: U.S. Geological Survey Miscellaneous Geologic Investigations Map I-610, 6 p., scale 1:125,000, 1 sheet.

Post, Austin, and Mayo, L.R., 1971, Glacier dammed lakes and outburst floods in Alaska: U.S. Geological Survey Hydrologic Investigations Atlas HA-455, 9 p., scale 1:1,000,000, 3 sheets.

\section{Bethel}

Dean, K.G., 1984a, Stream-icing zones in Alaska: Alaska Division of Geological \& Geophysical Surveys Report of Investigations 84-16, 20 p., scale 1:250,000, 102 sheets.

Hoare, J.M., and Coonrad, W.L., 1959a, Geology of the Bethel Quadrangle, Alaska: U.S. Geological Survey Miscellaneous Geologic Investigations Map I-285, scale 1:250,000, 1 sheet. 
Plafker, George, Hudson, Travis, and Richter, D.H., 1977, Preliminary observations on late Cenozoic displacements along the Totschunda and Denali fault systems, in Blean, K.M., ed., The United States Geological Survey in Alaska: Accomplishments during 1976: U.S. Geological Survey Circular 75 I-B, p. B67-B69.

\section{Bettles}

Brogan, G.E., Cluff, L.S., Korringa, M.K., and Slemmons, D.B., 1975, Active faults in Alaska, in Pavoni, N., and Green, R., eds., Recent crustal movements: Tectonophysics, v.29, no. 1-4, p. 73-85.

Dean, K.G., 1984a, Stream-icing zones in Alaska: Alaska Division of Geological \& Geophysical Surveys Report of Investigations 84-16, 20 p., scale 1:250,000, 102 sheets.

Hamilton, T.D., 1984, Late Quaternary offsets along the Kobuk and related fault zones, northwestern Alaska (abs.): Geological Society of America Abstracts with Programs, v.16, no. 5 , p. 288.

Patton, W.W., Jr., 1973, Reconnaissance geology of the northern Yukon-Koyukuk province, Alaska: U.S. Geological Survey Professional Paper 774-A, 17 p.

Patton, W.W., Jr., and Miller, T.P., 1973, Bedrock geologic map of Bettles and southern part of Wiseman Quadrangles, Alaska: U.S. Geological Survey Miscellaneous Field Studies Map MF-492, scale 1:250,000, 1 sheet.

\section{Black River}

Brabb, E.E., 1970, Preliminary geologic map of the Black River Quadrangle, east-central Alaska: U.S. Geological Survey Miscellaneous Geologic Investigations Map I-601, scale 1:250,000, 1 sheet.

Dean, K.G., 1984a, Stream-icing zones in Alaska: Alaska Division of Geological \& Geophysical Surveys Report of Investigations 84-16, 20 p., scale 1:250,000, 102 sheets.

\section{Bradfield Canal}

Gehrels, G.E., and Berg, H.C., 1984, Geologic map of southeastern Alaska: U.S. Geological Survey Open-file Report 84-886, 28 p., scale 1:600,000, 1 sheet.

Post, Austin, and Mayo, L.R., 1971, Glacier dammed lakes and outburst floods in Alaska: U.S. Geological Survey Hydrologic Investigations Atlas HA-455, 9 p., scale 1:1,000,000, 3 sheets. 


\section{Candle}

Dean, K.G., 1984a, Stream-icing zones in Alaska: Alaska Division of Geological \& Geophysical Surveys Report of Investigations 84-16, 20 p., scale 1:250,000, 102 sheets.

Patton, W.W., Jr., 1967, Regional geologic map of the Candle Quadrangle, Alaska: U.S. Geological Survey Miscellaneous Geologic Investigations Map I-492, scale 1:250,000.1 sheet.

\section{Chandalar}

Brosgé, W.P., and Reiser, H.N., 1964, Geologic map and section of the Chandalar Quadrangle, Alaska: U.S. Geological Survey Miscellaneous Geologic Investigations Map I-375, scale 1:250,000, 1 sheet.

Dean, K.G., 1984a, Stream-icing zones in Alaska: Alaska Division of Geological \& Geophysical Surveys Report of Investigations 84-16, 20 p., scale 1:250,000, 102 sheets.

Hamilton, T.D., 1978, Surficial geologic map of the Chandalar, Quadrangle, Alaska: U.S. Geological Survey Miscellaneous Field Studies Map MF-878A, scale 1:250,000, 1 sheet.

Chandler Lake

Dean, K.G., 1984a, Stream-icing zones in Alaska: Alaska Division of Geological \& Geophysical Surveys Report of Investigations 84-16, 20 p., scale 1:250,000, 102 sheets.

Hamilton, T.D., 1979, Surficial geologic map of the Chandler Lake Quadrangle, Alaska: U.S. Geological Survey Miscellaneous Field Studies Map MF-I 121, scale 1:250,000, 1 sheet.

Kelley, J.S., 1990, Generalized geologic map of the Chandler Lake Quadrangle, north-central Alaska: U.S. Geological Survey Miscellaneous Field Studies Map MF-2144A, 19 p., scale 1:250,000, 1 sheet.

\section{Charley River}

Dean, K.G., 1984, Stream-icing zones in Alaska: Alaska Division of Geological \& Geophysical Surveys Report of Investigations 84-16, 20 p., scale 1:250,000, 102 sheets.

Dover, J.H., and Miyaoka, R.T., 1988, Reinterpreted geologic map and fossil data, Charley River Quadrangle, east-central Alaska: U.S. Geological Survey Miscellaneous Field Studies Map MF-2004, scale 1:250,000, 2 sheets.

Patton, W.W., Jr., 1973, Reconnaissance geology of the northern Yukon-Koyukuk province, Alaska: U.S. Geological Survey Professional Paper 774-A, 17 p. 


\section{Chignik}

Dean, K.G., 1984a, Stream-icing zones in Alaska: Alaska Division of Geological \& Geophysical Surveys Report of Investigations 84-16, 20 p., scale 1:250,000, 102 sheets.

Detterman, R.L., Miller, T.P., Yount, M.E., and Wilson, F.H., 1981a, Geologic map of the Chignik and Sutwik Island Quadrangles, Alaska: U.S Geological Survey Miscellaneous Investigations Map I-1229, scale 1:250,000, 1 sheet.

1981b, Quaternary geologic map of the Chignik and Sutwik Island Quadrangles, Alaska: U.S. Geological Survey Miscellaneous Investigations Map I-1292, scale 1:250,000, 1 sheet.

\section{Circle}

Foster, H.L., Laird, Jo, Keith, T.E.C., Cushing, G.W., and Menzie, W.D., 1983, Preliminary geologic map of the Circle Quadrangle, Alaska: U.S. Geological Survey Open-file Report 83-170A, 30 p., scale 1:250,000, 1 sheet.

Weber, F.R., and Foster, H.L., 1982, Tertiary(?) conglomerate and Quaternary faulting in the Circle Quadrangle, Alaska, in Coonrad, W.L., ed., The United States Geological Survey in Alaska: Accomplishments during 1980: U.S. Geological Survey Circular 844, p. 58-60.

Yeend, Warren, 1989, Late Cenozoic sedimentary history along major fault zones, Alaska, in Carter, L.D., Hamilton, T.D., and Galloway, J.P., eds., Late Cenozoic history of the interior basins of Alaska and the Yukon: U.S. Geological Survey Circular 1026, p. 55-59.

\section{Cold Bay}

Dean, K.G., 1984a, Stream-icing zones in Alaska: Alaska Division of Geological \& Geophysical Surveys Report of Investigations 84-16, 20 p., scale 1:250,000, 102 sheets.

DuBois, G.D., Wilson, F.H., Detterman, R.L., and Hopkins, R.T., Jr., 1989, Reconnaissance geology and exploration geochemistry of King Cove, Alaska Peninsula: U.S. Geological Survey Open-file Report 89-350, 23 p.

McLean, Hugh, Engelhardt, C.L., and Howell, D.G., 1978, Reconnaissance geologic map of the Cold Bay and False Pass Quadrangles, Alaska: U.S. Geological Survey Open-file Report 78-323, scale 1:250,000, 1 sheet.

Waldron, H.H., 1961, Geologic reconnaissance of Frosty Peak Volcano and vicinity, Alaska: U.S. Geological Survey Bulletin 1028T, p. 677-708, scale 1:250,000, 1 sheet.

\section{Cordova}

Dean, K.G., 1984a, Stream-icing zones in Alaska: Alaska Division of Geological \& Geophysical Surveys Report f Investigations 84-16, 20 p., scale 1:250,000, 102 sheets. 
Kachadoorian, Reuben, 1960, Engineering geology of the Katalla area, Alaska: U.S. Geological Survey Miscellaneous Geologic Investigations Map I-308, scale 1:63,360, 1 sheet.

Nelson, S.W., Dumoulin, J.A., and Miller, M.L., 1985, Geologic map of the Chugach National Forest, Alaska: U.S. Geological Survey Miscellaneous Field Studies Map MF-1645B, 15 p., scale 1:250,000, 1 sheet.

Post, Austin, and Mayo, L.R., 1971, Glacier dammed lakes and outburst floods in Alaska: U.S. Geological Survey Hydrologic Investigations Atlas HA-455, 9 p., scale 1:1,000,000, 3 sheets.

Tysdal, R.G., Hudson, Travis, and Plafker, George, 1976, Surface features and recent movement along the Ragged Mountain fault, southcentral Alaska: U.S. Geological Survey Miscellaneous Field Studies Map MF-782, scale 1:24,000, 1 sheet.

Winkler, G.R., and Plafker, George, 1981, Geologic map and cross sections of the Cordova and Middleton Island Quadrangles, southern Alaska: U.S. Geological Survey Open-file Report 81-1164, 25 p., scale 1:250,000, 1 sheet.

\section{De Long Mountains}

Dean, K.G., 1984a, Stream-icing zones in Alaska: Alaska Division of Geological \& Geophysical Surveys Report of Investigations 84-16, 20 p., scale 1:250,000, 102 sheets.

Ellersieck, Inyo, Curtis, S.M., Mayfield, C.F., and Tailleur, I.L., 1990, Reconnaissance geologic map of the DeLong Mountains A-2 and B-2 Quadrangles and part of the C-2 Quadrangle, Alaska: U.S. Geological Survey Miscellaneous Investigations Map I-1931, scale 1:63,360, 2 sheets.

Mayfield, C.F., Curtis, S.M., Ellersieck, Inyo, and Tailleur, I.L., 1990, Reconnaissance geologic map of the DeLong Mountains A-3 and B-3 Quadrangles and parts of the A-4 and B-4 Quadrangles, Alaska: U.S. Geological Survey Miscellaneous Investigations Map I-1929, scale $1: 63,360,2$ sheets.

Mull, C.G., unpublished data, Compilation of bedrock geology of DeLong Mountains Quadrangle, Alaska: scale 1:250,000, 1 sheet.

Sable, E.G., Chapman, R.M., and Tailleur, I.L., 1984, Geologic map of the western KukpowrukNuka Rivers region, northwestern Alaska: U.S. Geological Survey Miscellaneous Field Studies Map MF-1668, scale 1:63,360, 2 sheets.

Sable, E.G., and Mangus, M.D., 1984, Geologic map of the west-central Kukpowruk-Nuka Rivers region, northwestern Alaska: U.S. Geological Survey Miscellaneous Field Studies Map MF-1669, scale 1:63,360, 2 sheets. 


\section{Demarcation Point}

Dean, K.G., 1984a, Stream-icing zones in Alaska: Alaska Division of Geological \& Geophysical Surveys Report of Investigations 84-16, 20 p., scale 1:250,000, 102 sheets.

Reiser, H.N., Brosgé, W.P., Dutro, J.T., Jr., and Detterman, R.L., 1980, Geologic map of the Demarcation Point Quadrangle, Alaska: U.S. Geological Survey Miscellaneous Investigations Map I-1133, scale 1:250,000, 1 sheet.

\section{Dillingham}

Beikman, H.M., 1974, Preliminary geologic map of the southwest quadrant of Alaska: U.S. Geological Survey Miscellaneous Field Studies Map MF-61 1, scale 1:1,000,000, 2 sheets.

Dean, K.G., 1984a, Stream-icing zones in Alaska: Alaska Division of Geological \& Geophysical Surveys Report of Investigations 84-16, 20 p., scale 1:250,000, 102 sheets.

Mertie, J.B., Jr., 1938, The Nushagak district, Alaska: U.S Geological Survey Bulletin 903, 96 p., scale $1: 250,000,2$ sheets.

\section{Eagle}

Foster, H.L., 1976, Geologic map of the Eagle Quadrangle, Alaska: U.S. Geological Survey Miscellaneous Investigations Map I-922, scale 1:250,000, 1 sheet.

Patton, W.W., Jr., 1973, Reconnaissance geology of the northern Yukon-Koyukuk province, Alaska: U.S. Geological Survey Professional Paper 774-A, 17 p.

\section{Fairbanks}

Combellick, R.A., 1984, Potential for earthquake-induced liquefaction in the Fairbanks-Nenana area, Alaska: Alaska Division of Geological \& Geophysical Surveys Report of Investigations $84-5,10$ p., scale 1:250,000, 1 sheet.

Péwé, T.L., Wahrhaftig, Clyde, and Weber, Florence, 1966, Geologic map of the Fairbanks Quadrangle, Alaska: U.S. Geological Survey Miscellaneous Geological Investigations Map I-455, 5 p., scale 1:250,000, 1 sheet.

Pulpan, Hans, 1988, Seismic-hazard analysis of the Nenana agricultural development area, central Alaska: Alaska Division of Geological \& Geophysical Surveys., Report of Investigations 88-1, 29 p.

Rawlinson, S.E., 1987, Preliminary photointerpretive maps of the geology, geologic materials, permafrost, and wetlands classification, Fairbanks B-5 Quadrangle, Alaska: Alaska Division of Geological \& Geophysical Surveys Public-data File 87-17, 26 p., scale 1:250,000, 4 sheets. 
Reger, R.D., 1987a, Preliminary photointerpretive maps of the geology, geologic materials, permafrost, and wetlands classification, Fairbanks C-5 Quadrangle, Alaska: Alaska Division of Geological \& Geophysical Surveys Public-data File 87-19, 22 p., scale $1: 31,680,4$ sheets.

1987b, Preliminary photointerpretive maps of the geology, geologic materials, permafrost, and wetlands classification, Fairbanks C-6 Quadrangle, Alaska: Alaska Division of Geological \& Geophysical Surveys Public-data File 87-20, 16 p., scale 1:31,680, 4 sheets.

Flaxman Island

Carter, L.D., Ferrians, 0.J., Jr., and Galloway, J.P., 1986, Engineering-geologic maps of northern Alaska, coastal plain and foothills of the Arctic National Wildlife Refuge: U.S. Geological Survey Open-file Report 86-334, 9 p., scale 1:250,000, 2 sheets.

Dean, K.G., 1984a, Stream-icing zones in Alaska: Alaska Division of Geological \& Geophysical Surveys Report of Investigations 84-16, 20 p., scale 1:250,000, 102 sheets.

\section{Harrison Bay}

Carter, L.D., and Galloway, J.P., 1985, Engineering-geologic maps of northern Alaska, Harrison Bay Quadrangle: U.S. Geological Survey Open-file Report 85-256, 47 p., scale 1:250,000, 2 sheets.

Rawlinson, S.E., 1990, Surficial geology and morphology of the Alaskan central Arctic Coastal Plain: Fairbanks, University of Alaska PhD dissertation, 311 p., scale 1:63,360, 6 sheets.

\section{Healy}

Brogan, G.E., Cluff, L.S., Korriga, M.K., and Slemmons, D.B., 1975, Active faults in Alaska, in Pavoni, N., and Green, R., eds., Recent crustal movements: Tectonophysics, v.29, no. 1-4, p. 73-85.

Csejtey, Bela, Jr., Mullen, M.W., Cox, D.P., Gilbert, W.G., Yeend, W.E., Smith, T.E., Wahrhaftig, Clyde, Craddock, Campbell, Brewer, W.M., Sherwood, K.W., Hickman, R.G., Stricker, G.D., St. Aubin, D.R., and Goerz, D.J., III, 1986, Geology and geochronology of the Healy Quadrangle, Alaska: U.S. Geological Survey Open-file Report 86-396, 96 p., scale 1:250,000, 4 sheets.

Dean, K.G., 1984a, Stream-icing zones in Alaska: Alaska Division of Geological \& Geophysical Surveys Report of Investigations 84-16, 20 p., scale 1:250,000, 102 sheets.

Gilbert, W.G., and Bundtzen, T.K., 1976, General geology and geochemistry of Healy D-5 and D-6 Quadrangles, Alaska: Alaska Division of Geological \& Geophysical Surveys Open-file Report 101, 6 p., scale 1:63,360, 2 sheets. 
Post, Austin, and Mayo, L.R., 1971, Glacier dammed lakes and outburst floods in Alaska: U.S. Geological Survey Hydrologic Investigations Atlas HA-455, 9 p., scale 1:1,000,000, 3 sheets.

Thorson, R.M., 1979, Recurrent late Quaternary faulting near Healy, Alaska, in Short Notes on Alaskan Geology: Alaska Division of Geological \& Geophysical Surveys Geologic Report 61, p. 10-14.

Wahrhaftig, Clyde, 1970a, Geologic map of the Healy D4 Quadrangle, Alaska: U.S. Geological Survey Geologic Quadrangle Map GQ-806, scale 1:63,360, 1 sheet.

1970b, Geologic map of the Healy D-5 Quadrangle, Alaska: U.S. Geological Survey Geologic Quadrangle Map GQ-807, scale 1:63,360, 1 sheet.

\section{Holy Cross}

Beikman, H.M., 1974, Preliminary geologic map of the southwest quadrant of Alaska: U.S. Geological Survey Miscellaneous Field Studies Map MF-61 1, scale 1:1,000,000, 2 sheets.

Dean, K.G., 1984a, Stream-icing zones in Alaska: Alaska Division of Geological \& Geophysical Surveys Report of Investigations 84-16, 20 p., scale 1:250,000, 102 sheets.

Livingston, H.R., 1983, Holy Cross Airport (Project F25341): Fairbanks, Alaska Department of Transportation and Public Facilities engineering geology and soils report, $11 \mathrm{p}$.

\section{Howard Pass}

Dean, K.G., 1984a, Stream-icing zones in Alaska: Alaska Division of Geological \& Geophysical Surveys Report of Investigations 84-16, 20 p., scale 1:250,000, 102 sheets.

Hamilton, T.D., 1984, Surficial geologic map of the Howard Pass Quadrangle, Alaska: U.S. Geological Survey Miscellaneous Field Studies Map MF-1677, scale 1:250,000, 1 sheet.

Mull, C.G., unpublished data, Compilation of bedrock geology of Howard Pass Quadrangle, Alaska: scale 1:250,000, 1 sheet.

Hughes

Dean, K.G., 1984a, Stream-icing zones in Alaska: Alaska Division of Geological \& Geophysical Surveys Report of Investigations 84-16, 20 p., scale 1:250,000, 102 sheets.

Hamilton, T.D., 1984, Late Quaternary offsets along the Kobuk and related fault zones, northwestern Alaska (abs.): Geological Society of America Abstracts with Programs, v.16, no. 5 , p. 288. 
Patton, W.W., Jr., 1973, Reconnaissance geology of the northern Yukon-Koyukuk province, Alaska: U.S. Geological Survey Professional Paper 774-A, 17 p.

Patton, W.W., Jr., and Miller, T.P., 1966, Regional geologic map of the Hughes Quadrangle, Alaska: U.S. Geological Survey Miscellaneous Geologic Investigations Map I-459, scale $1: 250,000,1$ sheet.

\section{Iditarod}

Bundtzen, T.K., and Laird, G.M., 1982, Geologic map of the Iditarod D-2 and eastern D-3 Quadrangles, Alaska: Alaska Division of Geological \& Geophysical Surveys Geologic Report 72, scale 1:63,360, 1 sheet.

1983a, Geologic map of the Iditarod D-1 Quadrangle, Alaska: Alaska Division of Geological \& Geophysical Surveys Professional Report 78, scale 1:63,360, 1 sheet.

Bundtzen, T.K., Laird, G.M., and Lockwood, M.S., 1988, Geologic map of the Iditarod C-3 Quadrangle, Alaska: Alaska Division of Geological \& Geophysical Surveys Professional Report 96, 13 p., scale 1:63,360, 1 sheet.

Bundtzen, T.K., Miller, M.L., Laird, G.M., and Bull, K.F., 1992, Geology and mineral resources of the Iditarod mining district, Iditarod B-4 and eastern B-5 Quadrangles, southwestern Alaska: Alaska Division of Geological \& Geophysical Surveys Professional Report 97, 46 p., scale 1:63,360, 2 sheets.

Cady, W.M., Wallace, R.E., Hoare, J.M., and Webber, E.J., 1955, The central Kuskokwim region, Alaska: U.S. Geological Survey Professional Paper 268, 132 p., scale 1:200,000, 5 sheets.

Dean, K.G., 1984a, Stream-icing zones in Alaska: Alaska Division of Geological \& Geophysical Surveys Report of Investigations 84-16, 20 p., scale 1:250,000, 102 sheets.

Miller, M.L., and Bundtzen, T.K., 1988, Right-lateral offset solution for the Iditarod-Nixon Fork fault, western Alaska, in Galloway, J.P., and Hamilton, T.D., eds., Geologic studies in Alaska by the U.S. Geological Survey during 1987: U.S. Geological Survey Circular 1016, p. 99-103.

in press, Simplified geologic map of the Iditarod Quadrangle and K-Ar, major oxide, fossil, paleocurrent, and archeological data: U.S. Geological Survey Miscellaneous field studies map.

Ikpikpuk River

Carter, L.D., and Galloway, J.P., 1988, Engineering-geologic maps of northern Alaska, Ikpikpuk River Quadrangle: U.S. Geological Survey Open-file Report 88-375, 19 p., scale 1:250,000, 2 sheets. 
Iliamna

Dean, K.G., 1984a, Stream-icing zones in Alaska: Alaska Division of Geological \& Geophysical Surveys Report of Investigations 84-16, 20 p., scale 1:250,000, 102 sheets.

Detterman, R.L., and Reed, B.L., 1973, Surficial deposits of the Iliamna Quadrangle, Alaska: U.S. Geological Survey Bulletin 1368A, 64 p., scale 1:250,000, 1 sheet.

Detterman, R.L., and Reed, B.L., 1980, Stratigraphy, structure, and economic geology of the Iliamna Quadrangle, Alaska: U.S. Geological Survey Bulletin 1368B, 86 p., scale $1: 250,000,1$ sheet.

Magoon, L.B., Adkison, W.L., and Egbert, R.M., 1976, Map showing geology, wildcat wells, Tertiary plant fossil localities, K-Ar age dates, and petroleum operations, Cook Inlet area, Alaska: U.S. Geological Survey Miscellaneous Investigations Map I-1019, scale 1:250,000, 3 sheets.

\section{Juneau}

Beikman, H.M., 1975, Preliminary geologic map of southeastern Alaska: U.S. Geological Survey Miscellaneous Field Studies Map MF-673, scale 1:1,000,000, 2 sheets.

Plafker, George, Hudson, Travis, and Bruns, Terry, 1978, Late Quaternary offsets along the Fairweather fault and crustal plate interactions in southern Alaska: Canadian Journal of Earth Sciences, v.15, no. 5, p. 805-816.

Post, Austin, and Mayo, L.R., 1971, Glacier dammed lakes and outburst floods in Alaska: U.S. Geological Survey Hydrologic Investigations Atlas HA-455, 9 p., scale 1:1,000,000, 3 sheets.

\section{Kantishna River}

Chapman, R.M., Yeend, W.E., Brosgé, W.P., and Reiser, H.N., 1975, Preliminary geologic map of the Tanana and northeast part of the Kantishna River Quadrangles, Alaska: U.S. Geological Survey Open-file Report 75-337, scale 1:250,000, 1 sheet.

Chapman, R.M., Yeend, W.E., and Patton, W.W., Jr., 1975, Preliminary reconnaissance geologic map of the western half of Kantishna River Quadrangle, Alaska: U.S. Geological Survey Open-file Report 75-351, scale 1:250,000, 1 sheet.

Collins, F.R., 1985, Map showing a vegetated dune field in central Alaska: U.S. Geological Survey Miscellaneous Field Studies Map MF-1708, 20 p., scale 1:250,000, 1 sheet.

Dean, K.G., 1984a, Stream-icing zones in Alaska: Alaska Division of Geological \& Geophysical Surveys Report of Investigations 84-16, 20 p., scale 1:250,000, 102 sheets. 


\section{Karluk}

Dean, K.G., 1984a, Stream-icing zones in Alaska: Alaska Division of Geological \& Geophysical Surveys Report of Investigations 84-16, 20 p., scale 1:250,000, 102 sheets.

Detterman, R.L., Wilson, F.H., Yount, M.E., and Miller, T.P., 1987, Quaternary geologic map of the Ugashik, Bristol Bay, and western part of Karluk Quadrangles, Alaska: U.S. Geological Survey Miscellaneous Investigations Map I-1801, scale 1:250,000, 1 sheet.

\section{Kateel River}

Brogan, G.E., Cluff, L.S., Korringa, M.K., and Slemmons, D.B., 1975, Active faults in Alaska, in Pavoni, N., and Green, R., eds., Recent crustal movements: Tectonophysics, v.29, no. 1-4, p. 73-85.

Dean, K.G., 1984a, Stream-icing zones in Alaska: Alaska Division of Geological \& Geophysical Surveys Report of Investigations 84-16, 20 p., scale 1:250,000, 102 sheets.

Patton, W.W., Jr., 1966, Regional geology of the Kateel River Quadrangle, Alaska: U.S. Geological Survey Miscellaneous Geologic Investigations Map I-437, scale 1:250,000, 1 sheet.

Weber, F.R., and Péwé, T.L., 1970, Surficial and engineering geology of the central part of the Yukon-Koyukuk lowland, Alaska: U.S. Geological Survey Miscellaneous Geologic Investigations Map I-590, scale 1:125,000, 2 sheets.

\section{Killik River}

Chapman, R.M., Detterman, R.L., and Mangus, M.D., 1964, Geology of the Killik-Etivluk Rivers region, Alaska: U.S. Geological Survey Professional Paper 303F, p. 325-407, scale $1: 125,000,2$ sheets, scale 1:250,000, 1 sheet.

Dean, K.G., 1984a, Stream-icing zones in Alaska: Alaska Division of Geological \& Geophysical Surveys Report of Investigations 84-16, 20 p., scale 1:250,000, 102 sheets.

Hamilton, T.D., 1980, Surficial geologic map of the Killik River Quadrangle, Alaska: U.S. Geological Survey Miscellaneous Field Studies Map MF-1234, scale 1:250,000, 1 sheet.

\section{Lake Clark}

Magoon, L.B., Adkison, W.L., and Egbert, R.M., 1976, Map showing geology, wildcat wells, Tertiary plant fossil localities, K-Ar age dates, and petroleum operations, Cook Inlet area Alaska: U.S. Geological Survey Miscellaneous Investigations Map I-1019, scale 1:250,000, 3 sheets. 
Nelson, W.H., Carlson, Christine, and Case, J.E., 1983, Geologic map of the Lake Clark Quadrangle, Alaska: U.S. Geological Survey Miscellaneous Field Studies Map MF-1114A, scale 1:250,000, 1 sheet.

\section{Lime Hills}

Capps, S.R., 1930, The Chakachamna-Stony region, Alaska: U.S. Geological Survey Bulletin 813B, p. 97-123.

Dean, K.G., 1984a, Stream-icing zones in Alaska: Alaska Division of Geological \& Geophysical Surveys Report of Investigations 84-16, 20 p., scale 1:250,000, 102 sheets.

DGGS, unpublished data, Compilation of geology of the Lime Hills Quadrangle: scale 1:250,000, 1 sheet.

Gilbert, W.G., 1981, Preliminary geologic map and geochemical data, Cheeneetnuk River area, Alaska: Alaska Division of Geological \& Geophysical Surveys Open-file Report 153, scale 1:63,360, 1 sheet.

Gilbert, W.G., Bundtzen, T.K., Kline, J.T., and Laird, G.M., 1990, Preliminary geology and geochemistry of the southwest part of the Lime Hills D-4 Quadrangle, Alaska: Alaska Division of Geological \& Geophysical Surveys Report of Investigations 90-6, scale 1:63,360, 1 sheet.

Post, Austin, and Mayo, L.R., 1971, Glacier dammed lakes and outburst floods in Alaska: U.S. Geological Survey Hydrologic Investigations Atlas HA-455, 9 p., scale 1:1,000,000, 3 sheets.

Weber, F.R., 196 1, Reconnaissance engineering geology for selection of highway route from Talkeetna to McGrath, Alaska: U.S. Geological Survey Open-file Report 61-169, scale 1:250,000, 15 sheets.

\section{Livengood}

Chapman, R.M., Weber, F.R., and Taber, Bond, 1971, Preliminary geologic Map of the Livengood Quadrangle, Alaska: U.S. Geological Survey Open-file Report 71-66, scale 1:250,000, 2 sheets.

Dean, K.G., 1984a, Stream-icing zones in Alaska: Alaska Division of Geological \& Geophysical Surveys Report of Investigations 84-16, 20 p., scale 1:250,000, 102 sheets.

\section{Lookout Ridge}

Yeend, Warren, 1983, Engineering-geologic maps of northern Alaska, Lookout Ridge Quadrangle: U.S. Geological Survey Open-file Report 83-279, scale 1:250,000, 2 sheets. 


\section{McGrath}

Blodgett, R.B., and Gilbert, W.G., 1983, The Cheeneetnuk Limestone, a new Early (?) to Middle Devonian Formation in the McGrath A-4 and A-5 Quadrangles, west-central Alaska: Alaska Division of Geological \& Geophysical Surveys Professional Report 85, 6 p., scale $1: 63,360,1$ sheet.

Bundtzen, T.K., Kline, J.T., and Clough, J.G., 1982, Preliminary geologic map of the McGrath B-2 Quadrangle, Alaska: Alaska Division of Geological \& Geophysical Surveys Open-file Report 149, 22 p., scale 1:40,000, 1 sheet.

Bundtzen, T.K., and Laird, G.M., 1983b, Geologic map of the McGrath D-6 Quadrangle, Alaska: Alaska Division of Geological \& Geophysical Surveys Professional Report 79, scale $1: 63,360,1$ sheet.

Dean, K.G., 1984a, Stream-icing zones in Alaska: Alaska Division of Geological \& Geophysical Surveys Report of Investigations 84-16, 20 p., scale 1:250,000, 102 sheets.

DGGS, unpublished data, Compilation of geology of the McGrath Quadrangle, Alaska: scale $1: 250,000,1$ sheet.

Fernald, A.T., 1960, Geomorphology of the upper Kuskokwim region, Alaska: U.S. Geological Survey Bulletin 1071G, p. 191-279, scale 1:250,000, 2 sheets.

Gilbert, W.G., 1981, Preliminary geologic map and geochemical data, Cheeneetnuk River area, Alaska: Alaska Division of Geological \& Geophysical Surveys Open-file Report 153, scale 1:63,360, 1 sheet.

Gilbert, W.G., Solie, D.N., and Kline, J.T., 1988, Geologic map of the McGrath A-3 Quadrangle, Alaska: Alaska Division of Geological \& Geophysical Surveys Professional Report 92, scale 1:63,360, 2 sheets.

Gilbert, W.G., Solie, D.N., Kline, J.T., and Dickey, D.B., 1990, Geologic map of the McGrath B-3 Quadrangle, Alaska: Alaska Division of Geological \& Geophysical Surveys Professional Report 102, scale 1:63,360, 2 sheets.

Kline, J.T., Gilbert, W.G., and Bundtzen, T.K., 1986, Preliminary geologic map of the McGrath C-1 Quadrangle, Alaska: Alaska Division of Geological \& Geophysical Surveys Report of Investigations 86-25, scale 1:63,360, 1 sheet.

Miller, M.L., and Bundtzen, T.K., 1988, Right-lateral offset solution for the Iditarod-Nixon Fork fault, western Alaska, in Galloway, J.P., and Hamilton, T.D., eds., Geologic studies in Alaska by the U.S. Geological Survey during 1987: U.S. Geological Survey Circular 1016, p. 99-103. 
Plafker, George, Hudson, Travis, and Richter, D.H., 1977, Preliminary observations on late Cenozoic displacements along the Totschunda and Denali fault systems, in Blean, K.M., ed., The United States Geological Survey in Alaska: Accomplishments during 1976: U.S. Geological Survey Circular 751-B, p. B67-B69.

Reed, B.L., and Elliott, R.L., 1970, Reconnaissance geologic map, analyses of bedrock and stream sediment samples, and an aeromagnetic map of parts of the southern Alaska Range: U.S. Geological Survey Open-file Report 70-271, 145 p., scale 1:250,000, 1 sheet.

Weber, F.R., 1961, Reconnaissance engineering geology for selection of highway route from Talkeetna to McGrath, Alaska: U.S. Geological Survey Open-file Report 61-169, scale 1:250,000, 15 sheets.

\section{Meade River}

Williams J.R., 1983, Engineering-geologic maps of northern Alaska, Meade River Quadrangle: U.S. Geological Survey Open-file Report 83-294, 29 p., scale 1:250,000, 1 sheet.

Medfra

Dean, K.G., 1984a, Stream-icing zones in Alaska: Alaska Division of Geological \& Geophysical Surveys Report of Investigations 84-16, 20 p., scale 1:250,000, 102 sheets.

Fernald, A.T., 1960, Geomorphology of the upper Kuskokwim region, Alaska: U.S. Geological Survey Bulletin 1071G, p. 191-279, scale 1:250,000, 2 sheets.

Miller, M.L., and Bundtzen, T.K., 1988, Right-lateral offset solution for the Iditarod-Nixon Fork fault, western Alaska, in Galloway, J.P., and Hamilton, T.D., eds., Geologic studies in Alaska by the U.S. Geological Survey during 1987: U.S. Geological Survey Circular 1016, p. 99-103.

Patton, W.W., Jr., Moll, E.J., Dutro, J.T., Jr., Silberman, M.L., and Chapman, R.M., 1980, Preliminary geologic map of the Medfra Quadrangle, Alaska: U.S. Geological Survey Open-file Report 80-811A, scale 1:250,000, 1 sheet.

\section{Melozitna}

Patton, W.W., Jr., Miller, T.P., Chapman, R.M., and Yeend, Warren, 1978, Geologic map of the Melozitna Quadrangle, Alaska: U.S. Geological Survey Miscellaneous Investigations Map I-1071, scale 1:250,000, 1 sheet.

\section{Misheguk Mountain}

Sable, E.G., and Mangus, M.D., 1984, Geologic map of the west-central Kukpowruk-Nuka Rivers region, northwestern Alaska: U.S. Geological Survey Miscellaneous Field Studies Map MF-1669, scale 1:63,360, 2 sheets. 
Sable, E.G., Mangus, M.D., Morris, R.H., and Dutro, J.T., Jr., 1984, Geologic map of the eastcentral Kukpowruk-Nuka Rivers region, northwestern Alaska: U.S. Geological Survey Miscellaneous Field Studies Map MF-1670, scale 1:63,360, 2 sheets.

Sable, E.G., Dutro, J.T., Jr., Morris, R.H., and Tailleur, I.L., 1984, Geologic map of the eastern Kukpowruk-Nuka Rivers region, northwestern Alaska: U.S. Geological Survey Miscellaneous Field Studies Map MF-1671, scale 1:63,360, 2 sheets.

\section{Mount Katmai}

Magoon, L.B., Adkison, W.L., and Egbert, R.M., 1976, Map showing geology, wildcat wells, Tertiary plant fossil localities, K-Ar age dates, and petroleum operations, Cook Inlet area, Alaska: U.S. Geological Survey Miscellaneous Investigations Map I-1019, scale 1:250,000, 3 sheets.

Riehle, J.R., Detterman, R.L., Yount, M.E., and Miller, J.W., 1987, Preliminary geologic map of the Mount Katmai Quadrangle and portions, of the Afognak and Naknek Quadrangles, Alaska: U.S. Geological Survey Open-file Report 87-593, scale 1:250,000, 1 sheet.

\section{Mount McKinley}

Bundtzen, T.K., Smith, T.E., and Tosdal, R.M., 1976, Progress report: Geology and mineral deposits of the Kantishna Hills, Alaska: Alaska Division of Geological \& Geophysical Surveys Open-file Report 98, 79 p., scale 1:63,360, 2 sheets.

Dean, K.G., 1984a, Stream-icing zones in Alaska: Alaska Division of Geological \& Geophysical Surveys Report of Investigations 84-16, 20 p., scale 1:250,000, 102 sheets.

Plafker, George, Hudson, Travis, and Richter, D.H., 1977, Preliminary observations on late Cenozoic displacements along the Totschunda and Denali fault systems, in Blean, K.M., ed., The United States Geological Survey in Alaska: Accomplishments during 1976: U.S. Geological Survey Circular 751 -B, p. B67-B69.

Post, Austin, and Mayo, L.R., 1971, Glacier dammed lakes and outburst floods in Alaska: U.S. Geological Survey Hydrologic Investigations Atlas HA-455, 9 p., scale 1:1,000,000, 3 sheets.

Reed, J.C., Jr., 1961, Geology of the Mount McKinley Quadrangle, Alaska: U.S. Geological Survey Bulletin 1108A, 36 p., scale 1:250,000, 1 sheet.

Ten Brink, N.W., 1984, North Alaska Range Project: Final report on 1978-1982 geoarcheological studies: report to National Geographic Society and National Park Service, 856 p., scale $1: 63,360,11$ sheets. 


\section{Mount Michelson}

Carter, L.D., Ferrians, 0.J., Jr., and Galloway, J.P., 1986, Engineering-geologic maps of northern Alaska, coastal plain and foothills of the Arctic National Wildlife Refuge: U.S. Geological Survey Open-file Report 86-334, 9 p., scale 1:250,000, 2 sheets.

Dean, K.G., 1984a, Stream-icing zones in Alaska: Alaska Division of Geological \& Geophysical Surveys Report of Investigations; 84-16, 20 p., scale 1:250,000, 102 sheets.

Rawlinson, S.E., 1990, Surficial geology and morphology of the Alaska central Arctic Coastal Plain: Fairbanks, University of Alaska PhD dissertation, 311 p., scale 1:63,360, 6 sheets.

Naknek

Dean, K.G., 1984a, Stream-icing zones in Alaska: Alaska Division of Geological \& Geophysical Surveys Report of Investigations 84-16, 20 p., scale 1:250,000, 102 sheets.

Ottley, Tom, 1982, King Salmon East Apron materials investigation (Project D21121): Anchorage, Alaska Department of Transportation and Public Facilities engineering geology and soils report, $58 \mathrm{p}$.

Riehle, J.R., Detterman, R.L., Yount, M.E., and Miller, J.W., 1987, Preliminary geologic map of the Mount Katmai Quadrangle and portions of the Afognak and Naknek Quadrangles, Alaska: U.S. Geological Survey Open-file Report 87-593, scale 1:250,000, 1 sheet.

\section{Noatak}

Beikman, H.M., and Lathram, E.H., 1976, Preliminary geologic map of northern Alaska: U.S. Geological Survey Miscellaneous Field Studies Map MF-789, scale 1:1,000,000, 2 sheets.

Dean, K.G., 1984a, Stream-icing zones in Alaska: Alaska Division of Geological \& Geophysical Surveys Report of Investigations 84-16, 20 p., scale 1:250,000, 102 sheets.

Krause, K., 1985, Noatak Quadrangle engineering geology: Alaska Division of Geological \& Geophysical Surveys Public-data File Report 8542A, scale 1:250,000, 1 sheet.

\section{Norton Bay}

Cass, J.T., 1959a, Reconnaissance geologic map of the Norton Bay Quadrangle: U.S. Geological Survey Miscellaneous Geologic Investigations Map I-286, scale 1:250,000, 1 sheet.

Dean, K.G., 1984a, Stream-icing zones in Alaska: Alaska Division of Geological \& Geophysical Surveys Report of Investigations 84-16, 20 p., scale 1:250,000, 102 sheets. 
Patton, W.W., Jr., 1973, Reconnaissance geology of the northern Yukon-Koyukuk province, Alaska: U.S. Geological Survey Professional Paper 774-A, 17 p.

Patton, W.W., Jr., and Hoare, J.M., 1968, The Kaltag fault, west-central Alaska, in Geological Survey research, 1968: U.S. Geological Survey Professional Paper 600-D, p. D147-D153.

Riehle, J.R., Emmel, K.S., and Bolm, J.G., 1981, Reconnaissance report on surficial geology of coastal area from Tolstoi Point to Point Nome, Norton Sound, Alaska: Alaska Division of Geological \& Geophysical Surveys Open-file Report 125, 24 p., scale 1:63,360, 3 sheets.

Nulato

Bickel, R.S., and Patton, W.W., Jr., 1957, Preliminary geologic map of the Nulato and Kateel Rivers area, Alaska: U.S. Geological Survey Miscellaneous Geologic Investigations Map I249, scale 1:125,000, 1 sheet.

Cass, J.T., 1959b, Reconnaissance geologic map of the Nulato Quadrangle, Alaska: Miscellaneous Geologic Investigations Map I-291, scale 1:250,000.1 sheet.

Dean, K.G., 1984a, Stream-icing zones in Alaska: Alaska Division of Geological \& Geophysical Surveys Report of Investigations 84-16, 20 p., scale 1:250,000, 102 sheets.

Martinson, M.A., 1982, Centerline soils and materials sources, Kaltag Airport (Project D19921): Anchorage, Alaska Department of Transportation and Public Facilities engineering geology and soils report, $7 \mathrm{p}$.

Patton, W.W., Jr., 1973, Reconnaissance geology of the northern Yukon-Koyukuk province, Alaska: U.S. Geological Survey Professional Paper 774-A, 17 p.

Patton, W.W., Jr., and Hoare, J.M., 1968, The Kaltag fault, west-central Alaska, in Geological Survey research, 1968: U.S. Geological Survey Professional Paper 600-D, p. D147-D153.

Weber, F.R., and Péwé, T.L., 1971, Surficial and engineering geology of the central part of the Yukon-Koyukuk lowland, Alaska: U.S. Geological Survey Miscellaneous Geologic Investigations Map I-590, scale 1:125,000, 2 sheets.

\section{Ophir}

Bundtzen, T.K., and Laird, G.M., 1980, Preliminary geology of the McGrath-Innoko River area, western interior Alaska: Alaska Division of Geological \& Geophysical Surveys Open-file Report 134, 36 p., scales 1:63,360 and 1:125,000, 2 sheets.

Chapman, R.M., Patton, W.W., Jr., and Moll, E.J., 1985, Reconnaissance geologic map of the Ophir Quadrangle, Alaska: U.S. Geological Survey Open-file Report 85-203, 17 p., scale $1: 250,000,1$ sheet. 


\section{Petersburg}

Brew, D.A., Ovenshine, A.T., Karl, S.M., and Hunt, S.J., 1984, Preliminary reconnaissance geologic map of the Petersburg and parts of the Port Alexander and Sumdum 1:250,000 Quadrangles, southeastern Alaska: U.S. Geological Survey Open-file Report 84-405, 43 p., scale $1: 250,000,2$ sheets.

Post, Austin, and Mayo, L.R., 1971, Glacier dammed lakes and outburst floods in Alaska: U.S. Geological Survey Hydrologic Investigations Atlas HA-455, 9 p., scale 1:1,000,000, 3 sheets.

\section{Philip Smith Mountains}

Brosgé, W.P., Reiser, H.N., Dutro, J.T., Jr., and Detterman, R.L., 1979, Bedrock geologic map of the Philip Smith Mountains Quadrangle, Alaska: U.S. Geological Survey Miscellaneous Field Studies Map MF-879B, scale 1:250,000, 2 sheets.

Dean, K.G., 1984a, Stream-icing zones in Alaska: Alaska Division of Geological \& Geophysical Surveys Report of Investigations 84-16, 20 p., scale 1:250,000, 102 sheets.

Hamilton, T.D., 1978, Surficial geologic map of the Philip Smith Mountains Quadrangle, Alaska: U.S. Geological Survey Miscellaneous Field Studies Map MF-879A, scale 1:250,000, 1 sheet.

\section{Port Moller}

Dean, K.G., 1984a, Stream-icing zones in Alaska: Alaska Division of Geological \& Geophysical Surveys Report of Investigations 84-16, 20 p., scale 1:250,000, 102 sheets.

Wilson, F.H., Detterman, R.L., and Harris, E.E., 1991, Generalized geologic map of the Port Moller, Stepovak Bay, and Simeonof Island Quadrangles, Alaska Peninsula, Alaska: U.S. Geological Survey Miscellaneous Field Studies Map MF-2155A, scale 1:250,000, 1 sheet.

Ruby

Cass, J.T., 1959c, Reconnaissance geologic map of the Ruby Quadrangle, Alaska: U.S. Geological Survey Miscellaneous Geologic Investigations Map I-289, scale 1:63,360, 1 sheet.

Dean, K.G., 1984a, Stream-icing zones in Alaska: Alaska Division of Geological \& Geophysical Surveys Report of Investigations 84-16, 20 p., scale 1:250,000, 102 sheets.

Patton, W.W., Jr., 1973, Reconnaissance geology of the northern Yukon-Koyukuk province, Alaska: U.S. Geological Survey Professional Paper 774-A, 17 p.

Patton, W.W., Jr., and Hoare, J.M., 1968, The Kaltag fault, west-central Alaska, in Geological Survey research, 1968: U.S. Geological Survey Professional Paper 600-D, p. D147-D153. 


\section{Russian Mission}

Bundtzen, T.K., and Laird, G.M., 1991, Geology and mineral resources of the Russian Mission C-1 Quadrangle, southwest Alaska: Alaska Division of Geological \& Geophysical Surveys Professional Report 109, 24 p., scale 1:63,360, 2 sheets.

Cady, W.M., Wallace, R.E., Hoare, J.M., and Webber, E.J., 1955, The central Kuskokwim region, Alaska: U.S. Geological Survey Professional Paper 268, 132 p., scale 1:200,000, 5 sheets.

Dean, K.G., 1984a, Stream-icing zones in Alaska: Alaska Division of Geological \& Geophysical Surveys Report of Investigations 84-16, 20 p., scale 1:250,000, 102 sheets.

Hoare, J.M., and Coonrad, W.L., 1959b, Geology of the Russian Mission Quadrangle, Alaska: U.S. Geological Survey Miscellaneous Geologic Investigations Map I-292, scale 1:250,000, 1 sheet.

Miller, M.L., and Bundtzen, T.K., 1988, Right-lateral offset solution of the Iditarod-Nixon Fork fault, western Alaska, in Galloway, J.P., and Hamilton, T.D., eds., Geologic studies in Alaska by the U.S. Geological Survey during 1987: U.S. Geological Survey Circular 1016, p. $99-103$.

\section{Sagavanirktok}

Beikman, H.M., and Lathram, E.H., 1976, Preliminary geologic map of northern Alaska: U.S. Geological Survey Miscellaneous Field Studies Map MF-789, scale 1:1,000,000, 2 sheets.

Dean, K.G., 1984a, Stream-icing zones in Alaska: Alaska Division of Geological \& Geophysical Surveys Report of Investigations 84-16, 20 p., scale 1:250,000, 102 sheets.

Ferrians, O.J., Jr., 1971, Preliminary engineering geologic maps of the proposed trans-Alaska pipeline route, Beechey Point and Sagavanirktok Quadrangles: U.S. Geological Survey Open-file Report 71-101, scale 1:125,000, 2 sheets.

Yeend, Warren, 1973, Preliminary geologic map of a prospective transportation route from Prudhoe Bay, Alaska, to Canadian border, Part 1, Beechey Point and Sagavanirktok Quadrangles: U.S. Geological Survey Miscellaneous Field Studies Map MF-489, scale $1: 125,000,2$ sheets.

\section{Selawik}

Dean, K.G., 1984a, Stream-icing zones in Alaska: Alaska Division of Geological \& Geophysical Surveys Report of Investigations 84-16, 20 p., scale 1:250,000, 102 sheets. 
Hamilton, T.D., 1984, Late Quaternary offsets along the Kobuk and related fault zones, northwestern Alaska (abs.): Geological Society of America Abstracts with Programs, v.16, no. 5 , p. 288.

Patton, W.W., Jr., 1973, Reconnaissance geology of the northern Yukon-Koyukuk province, Alaska: U.S. Geological Survey Professional Paper 774-A, 17 p.

Patton, W.W., Jr., and Miller, T.P., 1968, Regional geologic map of the Selawik and southeastern Baird Mountains Quadrangles, Alaska: U.S. Geological Survey Miscellaneous Geologic Investigations Map I-530, scale 1:250,000, 1 sheet.

\section{Shungnak}

Brogan, G.E., Cluff, L.S., Korringa, M.K., and Slemmons, D.B., 1975, Active faults in Alaska, in Pavoni, N., and Green, R., eds., Recent crustal movements: Tectonophysics, v.29, no. 1-4, p. 73-85.

Dean, K.G., 1984a, Stream-icing zones in Alaska: Alaska Division of Geological \& Geophysical Surveys Report of Investigations 84-16, 20 p., scale 1:250,000, 102 sheets.

Fernald, A.T., 1964, Surficial geology of the central Kobuk River valley, northwestern Alaska: U.S. Geological Survey Bulletin 1181K, 31 p., scale 1:250,000, 1 sheet.

Fritts, C.E., 1969, Geology and geochemistry of the southeastern part of the Cosmos Hills, Shungnak D-2 Quadrangle, Alaska: Alaska Division of Mines and Geology Geologic Report 37, $35 \mathrm{p}$.

1970, Geology and geochemistry of the Cosmos Hills, Ambler River and Shungnak Quadrangles, Alaska: Alaska Division of Mines and Geology Geologic Report 39, 69 p.

Hamilton, T.D., 1984, Late Quaternary offsets along the Kobuk and related fault zones, northwestern Alaska (abs.): Geological Society of America Abstracts with Programs, v.16, no. 5 , p. 288 .

Hitzman, M.W., Smith, T.E., and Proffett, J.M., 1982, Bedrock geology of the Ambler district, southwestern Brooks Range, Alaska: Alaska Division of Geological \& Geophysical Surveys Geologic Report 75, scale 1:125,000, 2 sheets.

Pallister, J.S., and Carlson, Christine, 1988, Bedrock geologic map of the Angayucham Mountains, Alaska: U.S. Geological Survey Miscellaneous Field Studies Map MF-2024, scale 1:63,360, 1 sheet.

Patton, W.W., Jr., 1973, Reconnaissance geology of the northern Yukon-Koyukuk province, Alaska: U.S. Geological Survey Professional Paper 774-A, 17 p. 
Patton, W.W., Jr., Miller, T.P., and Tailleur, I.L., 1968, Regional geologic map of the Shungnak and southern part of the Ambler River Quadrangles, Alaska: U.S. Geological Survey Miscellaneous Geologic Investigations Map I-554, scale 1:250,000, 1 sheet.

\section{Sleetmute}

Cady, W.M., Wallace, R.E., Hoare, J.M., and Webber, E.J., 1955, The central Kuskokwim region, Alaska: U.S. Geological Survey Professional Paper 268, 132 p., scale 1:200,000, 5 sheets.

Dean, K.G., 1984a, Stream-icing zones in Alaska: Alaska Division of Geological \& Geophysical Surveys Report of Investigations 84-16, 20 p., scale 1:250,000, 102 sheets.

Decker, John, Robinson, M.S., Murphy, J.M., Reifenstuhl, R.R., and Albanese, M.D., 1984, Geologic map of the Sleetmute A-6 Quadrangle, Alaska: Alaska Division of Geological \& Geophysical Surveys Report of Investigations 84-8, scale 1:40,000, 1 sheet.

Miller, M.L., Belkin, H.E., Blodgett, R.B., Bundtzen, T.K., Cady, J.W., Goldfarb, R.J., Gray, J.E., McGimsey, R.G., and Simpson, S.L., 1989, Pre-field study and mineral resource assessment of the Sleetmute Quadrangle, southwestern Alaska: U.S. Geological Survey Open-file Report 89-363, 115 p., scale 1:250,000, 3 sheets.

Miller, M.L., and Bundtzen, T.K., 1988, Right-lateral offset solution for the Iditarod-Nixon Fork fault, western Alaska, in Galloway, J.P., and Hamilton, T.D., eds., Geologic studies in Alaska by the U.S. Geological Survey during 1987: U.S. Geological Survey Circular 1016, p. 99-103.

Plafker, George, Hudson, Travis, and Richter, D.H., 1977, Preliminary observations on late Cenozoic displacements along the Totschunda and Denali fault systems, in Blean, K.M., ed., The United States Geological Survey in Alaska: Accomplishments during 1976: U.S. Geological Survey Circular 751-B, p. B67-B69.

Reifenstuhl, R.R., Robinson, M.S., Smith, T.E., Albanese, M.D., and Allegro, G.A., 1984, Geologic map of the Sleetmute B-6 Quadrangle, Alaska: Alaska Division of Geological \& Geophysical Surveys Report of Investigations 84-12, scale 1:40,000, 1 sheet.

Robinson, M.S., Decker, John, Reifenstuhl, R.R., Murphy, J.M., and Box, S.E., 1984, Geologic map of the Sleetmute B-5 Quadrangle, Alaska: Alaska Division of Geological \& Geophysical Surveys Report of Investigations 84-10, scale 1:40, 000, 1 sheet.

Waythomas, C.F., 1990, Quaternary geology and late Quaternary environments of the Holitna lowland and Chuilnuk-Kiokluk Mountains region, interior southwestern Alaska: Boulder, University of Colorado PhD dissertation, 268 p., scale 1:63,360, 1 sheet. 


\section{Solomon}

Dean, K.G., 1984, Stream-icing zones in Alaska: Alaska Division of Geological \& Geophysical Surveys Report of Investigations, 84-16, 20 p., scale 1:250,000, 102 sheets.

Kaufman, D.S., 1985, Windy Creek and Crater Creek faults, Seward Peninsula, in BartschWinkler, Susan and Reed, K.M., eds., The United States Geological Survey in Alaska: Accomplishments during 1983: U.S. Geological Survey Circular 945, p. 22-24.

1986, Surficial geologic map of the Solomon, Bendeleben, and southern part of the Kotzebue Quadrangle, western Alaska: U.S. Geological Survey Miscellaneous Field Studies Map MF-1838A, scale 1:250,000, 1 sheet.

Till, A.B., Dumoulin, J.A., Gamble, B.M., Kaufman, D.S., and Caroll, P.I., 1986, Preliminary geologic map and fossil data, Solomon, Bendeleben, and southern Kotzebue Quadrangles, Seward Peninsula, Alaska: U.S. Geological Survey Open-file Report 86-276, 60 p., scale $1: 250,000,3$ sheets.

\section{Survey Pass}

Dean, K.G., 1984a, Stream-icing zones in Alaska: Alaska Division of Geological \& Geophysical Surveys Report of Investigations 84-16, 20 p., scale 1:250,000, 102 sheets.

Hamilton, T.D., 1981, Surficial geologic map of the Survey Pass Quadrangle, Alaska: U.S. Geological Survey Miscellaneous Field Studies Map MF-1320, scale 1:250,000, 1 sheet.

Nelson, S.W., and Grybeck, Donald, 1980, Geologic map of the Survey Pass Quadrangle, Brooks Range, Alaska: U.S. Geological Survey Miscellaneous Field Studies Map MF-I 176A, scale $1: 250,000,2$ sheets.

\section{Taku River}

Beikman, H.M., 1975, Preliminary geologic map of southeastern Alaska: U.S. Geological Survey Miscellaneous Field Studies Map MF-673, scale 1:1,000,000, 2 sheets.

Brew, D.A., and Ford, A.B., 1985, Preliminary reconnaissance geologic map of the Juneau, Taku River, Atlin, and part of the Skagway 1:250,000 Quadrangles, southeastern Alaska: U.S. Geological Survey Open-file Report 85-395, 23 p., scale 1:250,000, 2 sheets.

Post, Austin, and Mayo, L.R., 1971, Glacier dammed lakes and outburst floods in Alaska: U.S. Geological Survey Hydrologic Investigations Atlas HA-455, 9 p., scale 1:1,000,000, 3 sheets.

Talkeetna 
Nelson, S.W., and Reed, B.L., 1978, Surficial deposits map of the Talkeetna Quadrangle, Alaska: U.S. Geological Survey Miscellaneous Field Studies Map MF-870J, scale 1:250,000, 1 sheet.

Plafker, George, Hudson, Travis, and Richter, D.H., 1977, Preliminary observations on late Cenozoic displacements along the Totschunda and Denali fault systems, in Blean, K.M., ed., The United States Geological Survey in Alaska: Accomplishments during 1976: U.S. Geological Survey Circular 751-B, p. B67-B69.

Reed, B.L., and Nelson, S.W., 1980, Geologic map of the Talkeetna Quadrangle, Alaska: U.S. Geological Survey Miscellaneous Investigations Map I-1 174, 15 p., scale 1:250,000, 1 sheet.

Tanana

Brogan, G.E., Cluff, L.S., Korringa, M.K., and Slemmons, D.B., 1975, Active faults in Alaska, in Pavoni, N., and Green, R., eds., Recent crustal movements: Tectonophysics, v.29, no. 1-4, p. 73-85.

Chapman, R.M., Yeend, Warren, Brosgé, W.P., and Reiser, H.N., 1982, Text and tables to accompany the reconnaissance geologic map of the Tanana Quadrangle, Alaska: U.S. Geological Survey Open-file Report 82-734, 18 p., scale 1:250,000, 1 sheet.

Dean, K.G., 1984a, Stream-icing zones in Alaska: Alaska Division of Geological \& Geophysical Surveys Report of Investigations 84-16, 20 p., scale 1:250,000, 102 sheets.

Patton, W.W., Jr., 1973, Reconnaissance geology of the northern Yukon-Koyukuk province, Alaska: U.S. Geological Survey Professional Paper 774-A, 17 p.

Patton, W.W., Jr., and Hoare, J.M., 1968, The Kaltag fault, west-central Alaska, in Geological Survey research, 1968: U.S. Geological Survey Professional Paper 600-D, p. D147-D153.

Yeend, Warren, 1989, Late Cenozoic sedimentary history along major fault zones, Alaska, in Carter, L.D., Hamilton, T.D., and Galloway, J.P., eds., Late Cenozoic history of the interior basins of Alaska and the Yukon: U.S. Geological Survey Circular 1026, p. 55-59.

\section{Taylor Mountains}

Beikman, H.M., 1974, Preliminary geologic map of the southwest quadrant of Alaska: U.S. Geological Survey Miscellaneous Field Studies Map MF-61 1, scale 1:1,000,000, 2 sheets.

Cady, W.M., Wallace, R.E., Hoare, J.M., and Webber, E.J., 1955, The central Kuskokwim region, Alaska: U.S. Geological Survey Professional Paper 268, 132 p., scale 1:200,000, 5 sheets.

DGGS, unpublished data, Compilation of geology of the Taylor Mountains Quadrangle, Alaska: scale $1: 1,000,000,1$ sheet. 
Plafker, George, Hudson, Travis, and Richter, D.H., 1977, Preliminary observations on late Cenozoic displacements along the Totschunda and Denali fault systems, in Blean, K.M., ed., The United States Geological Survey in Alaska: Accomplishments during 1976: U.S. Geological Survey Circular 751-B, p. B67-B69.

\section{Teshekpuk}

Carter, L.D., 1983, Engineering-geologic maps of northern Alaska, Teshekpuk Quadrangle: U.S. Geological Survey Open-file Report 83-634, scale 1:250,000, 1 sheet.

Dean, K.G., 1984a, Stream-icing zones in Alaska: Alaska Division of Geological \& Geophysical Surveys Report of Investigations 84-16, 20 p., scale 1:250,000, 102 sheets.

Tyonek

Barnes, F.F., 1966, Geology and coal resources of the Beluga-Yentna region, Alaska: U.S. Geological Survey Bulletin 1202C, 54 p., scales 1:63,360 and 1:250,000, 3 sheets.

Dean, K.G., 1984a, Stream-icing zones in Alaska: Alaska Division of Geological \& Geophysical Surveys Report of Investigations 84-16, 20 p., scale 1:250,000, 102 sheets.

Detterman, R.L., Plafker, George, Hudson, Travis, Tysdal, R.G., and Pavoni, Nazario, 1974, Surface geology and Holocene breaks along the Susitna segment of the Castle Mountain fault, Alaska: U.S. Geological Survey Miscellaneous Field Studies Map MF-618, scale $1: 24,000,1$ sheet.

Detterman, R.L., Hudson, Travis, and Hoare, J.M., 1975, Bruin Bay fault inactive during the Holocene, in Yount, M.E., ed., United States Geological Survey Alaska Program, 1975: U.S. Geological Survey Circular 722, p. 45.

Detterman, R.L., Hudson, Travis, Plafker, George, Tysdal, R.G., and Hoare, J.M., 1976, Reconnaissance geologic map along Bruin Bay and Lake Clark faults in Kenai and Tyonek Quadrangles, Alaska: U.S. Geological Survey Open-file Report 76-477, 4 p., scale 1:250,000, 1 sheet.

Hackett, S.W., 1977, Gravity survey of Beluga Basin and adjacent area, Cook Inlet region, southcentral Alaska: Alaska Division of Geological \& Geophysical Surveys Geologic Report 49, 26 p., scale 1:500,000, 3 sheets.

Magoon, L.B., Adkison, W.L., and Egbert, R.M., 1976, Map showing geology, wildcat wells, Tertiary plant fossil localities, K-Ar age dates, and petroleum operations, Cook Inlet area, Alaska: U.S. Geological Survey Miscellaneous Investigations Map I-1019, scale 1:250,000, 3 sheets. 
Odum, J.K., Yehle, L.A., Schmoll, H.R., Gardner, C.A., Dearborn, L.L., 1988, Lithological, geotechnical properties analysis, and geophysical log interpretation of U.S. Geological Survey Drill Holes 1C-79, 2C-80, CW 81-2, and CE 82-1, Tyonek Formation, upper Cook Inlet region, Alaska: U.S. Geological Survey Bulletin 1835, 27 p.

Post, Austin, and Mayo, L. R., 1971, Glacier dammed lakes and outburst floods in Alaska: U.S. Geological Survey Hydrologic Investigations Atlas HA 455, 9 p., scale 1:1,000,000, 3 sheets.

Rawlinson, S.E., Huck, R.W., and Hardy, S.B., 1982a, Peat-resource map, Tyonek D-1 Quadrangle, Alaska: Alaska Division of Geological \& Geophysical Surveys Open-file Report 150I, scale 1:31,680, 1 sheet.

1982b, Peat-resource map, Tyonek C-1 Quadrangle, Alaska: Alaska Division of Geological \& Geophysical Surveys Open-file Report 150K, scale 1:31,680, 1 sheet.

1982c, Peat-resource map, Tyonek B-1 Quadrangle, Alaska: Alaska Division of Geological \& Geophysical Surveys Open-file Report 150M, scale 1:31,680, 1 sheet.

Reger, R.D., 1978, Reconnaissance geology of the new capital site and vicinity, Anchorage Quadrangle, Alaska: Alaska Division of Geological \& Geophysical Surveys Open-file Report 113A, scale 1:63,360, 1 sheet.

Reger, R.D., and Updike, R.G., 1983, Upper Cook Inlet and Matanuska Valley, in Péwé, T.L., and Reger, R.D., eds., Guidebook to permafrost and Quaternary geology along the Richardson and Glenn Highways between Fairbanks and Anchorage, Alaska: Alaska Division of Geological \& Geophysical Surveys Guidebook 1, p. 185-263, scale 1:250,000, 1 sheet.

Schmoll, H.R., Chleborad, A.F., Yehle, L.A., and Gardner, C.A., 1981, Reconnaissance engineering geology of the Beluga coal resource area, southcentral Alaska, in Rao, P.D., and Wolff, E.N., eds., Focus on Alaska's coal '80: Fairbanks, University of Alaska Mineral Industries Research Laboratory Report 50, p. 92-110.

Schmoll, H.R., and Yehle, L.A., 1987, Surficial geologic map of the northwestern quarter of the Tyonek A-4 Quadrangle, south-central Alaska: U.S. Geological Survey Miscellaneous Field Studies Map MF-1934, scale 1:31,680, 1 sheet.

Solie, D.N., Gilbert, W.G., Harris, E.E., Kline, J.T., Liss, S.A., and Robinson, M.S., 1991, Preliminary geologic map of Tyonek D-6 and eastern Tyonek D-7 Quadrangles, Alaska: Alaska Division of Geological \& Geophysical Surveys Public-data File 91-10, 16 p., scale 1:63,360, 1 sheet.

Sturm, Matthew, 1986, Formation of a strandline during the 1984 jokulhlaup of Strandline Lake: Arctic, v.39, no. 3, p. 267-269. 
Sturm, Matthew, and Benson, C.S., 1989, Jokulhlaups from Strandline Lake, Alaska, with special attention to the 1982 event: Alaska Division of Geological \& Geophysical Surveys Report of Investigations 88-10, 19 p.

Weber, F.R., 1961, Reconnaissance engineering geology for selection of highway route from Talkeetna to McGrath, Alaska: U.S. Geological Survey Open-file Report 61-169, scale $1: 250,000,15$ sheets.

\section{Ugashik}

Detterman, R.L., Wilson, F.H., Yount, M.E., and Miller, T.P., 1987, Quaternary geologic map of the Ugashik, Bristol Bay, and western part of Karluk Quadrangles, Alaska: U.S. Geological Survey Miscellaneous Investigations Map I-1801, scale 1:250,000, 1 sheet.

\section{Umiat}

Carter, L.D., and Galloway, J.P., 1986, Engineering-geologic maps of northern Alaska, Umiat Quadrangle: U.S. Geological Survey Open-file Report 86-335, 16 p., scale 1:250,000, 2 sheets.

Dean, K.G., 1984a, Stream-icing zones in Alaska: Alaska Division of Geological \& Geophysical Surveys Report of Investigations 84-16, 20 p., scale 1:250,000, 102 sheets.

\section{Unalakleet}

Cass, J.T., 1959d, Reconnaissance geologic map of the Unalakleet Quadrangle, Alaska: U.S. Geological Survey Miscellaneous Geologic Investigations Map I-288, scale 1:250,000, 1 sheet.

Dean, K.G., 1984a, Stream-icing zones in Alaska: Alaska Division of Geological \& Geophysical Surveys Report of Investigations 84-16, 20 p., scale 1:250,000, 102 sheets.

Fisher, M.A., 1982, Preliminary interpretation of geophysical data over the projected offshore location of the Kaltag fault, in Coonrad, W.L., ed., The United States Geological Survey in Alaska: Accomplishments during 1980: U.S. Geological Survey Circular 844, p. 136-139.

Patton, W.W., Jr., 1973, Reconnaissance geology of the northern Yukon-Koyukuk province, Alaska: U.S. Geological Survey Professional Paper 774-A, 17 p.

Patton, W.W., Jr., and Hoare, J.M., 1968, The Kaltag fault, westcentral Alaska, in Geological Survey research, 1968: U.S. Geological Survey Professional Paper 600-D, p. D147-D153.

Patton, W.W., Jr., and Moll, E.J., 1985, Geologic map of northern and central parts of Unalakleet Quadrangle, Alaska: U.S. Geological Survey Miscellaneous Field Studies Map MF-1749, scale 1:250,000.1 sheet. 
Riehle, J.R., Emmel, K.S., and Bolm, J.G., 1981, Reconnaissance report on surficial geology of coastal area from Tolstoi Point to Cape Nome, Norton Sound, Alaska: Alaska Division of Geological \& Geophysical Surveys Open-file Report 125, 24 p., scale 1:63,360, 3 sheets.

\section{Utukok River}

Dean, K.G., 1984a, Stream-icing zones in Alaska: Alaska Division of Geological \& Geophysical Surveys Report of Investigations 84-16, 20 p., scale 1:250,000, 102 sheets.

Yeend, Warren, 1984, Engineering-geologic maps of northern Alaska, Utukok River Quadrangle: U.S. Geological Survey Open-file Report 84-682, scale 1:250,000, 2 sheets.

\section{Wiseman}

Dean, K.G., 1984, Stream-icing zones in Alaska: Alaska Division of Geological \& Geophysical Surveys Report of Investigations 84-16, 20 p., scale 1:250,000, 102 sheets.

Dillon, J.T., Brosgé, W.P., and Dutro, J.T., Jr., 1986, Generalized geologic map of the Wiseman Quadrangle, Alaska: U.S. Geological Survey Open-file Report 86-219, scale 1:250,000, 1 sheet.

Hamilton, T.D., 1979, Surficial geologic map of the Wiseman Quadrangle, Alaska: U.S. Geological Survey Miscellaneous Field Studies Map MF-1122, scale 1:250,000, 1 sheet. 


\section{APPENDIX 3}

TECHNICAL REFERENCES USED TO ASSESS ACTIVITY OF FAULTS IN QUADRANGLES TRAVERSED BY 10-MILE-WIDE PROPOSED CORRIDORS IN ALASKA

\begin{tabular}{|c|c|c|}
\hline Quadrangle & Fault & Reference $^{1}$ \\
\hline \multirow[t]{2}{*}{ Anchorage } & Border Ranges-Knik & $\begin{array}{l}\text { Burns and others (1991) } \\
\text { Magoon and others (1976) } \\
\text { Reger and Updike (1983) }\end{array}$ \\
\hline & Castle Mountain-Caribou & $\begin{array}{l}\text { Bruhn (1979) } \\
\text { Detterman and others }(1974, \\
1975,1976) \\
\text { Grantz (1961) } \\
\text { Lahr and Page }(1986) \\
\text { Lahr and others }(1985,1986) \\
\text { Magoon and others (1976) } \\
\text { Page and Lahr (1971) } \\
\text { Reger (1981c) } \\
\text { Reger and Updike (1983) } \\
\end{array}$ \\
\hline \multirow[t]{3}{*}{ Bendeleben } & Bendeleben & $\begin{array}{l}\text { Hudson and Plafker (1978) } \\
\text { Kaufman }(1985,1986) \\
\text { Till and others }(1986) \\
\end{array}$ \\
\hline & Kigluaik & $\begin{array}{l}\text { Hudson and Plafker (1978) } \\
\text { Kaufman }(1985,1986) \\
\text { Till and others }(1986)\end{array}$ \\
\hline & Windy Creek & $\begin{array}{l}\text { Kaufman }(1985,1986) \\
\text { Till and others }(1986) \\
\end{array}$ \\
\hline Bethel & Milk Creek-Holitna & $\begin{array}{l}\text { Hoare and Coonrad (1959a) } \\
\text { Plafker and others (1977) }\end{array}$ \\
\hline \multirow[t]{2}{*}{ Bettles } & Kanuti & $\begin{array}{l}\text { Brogan and others (1975) } \\
\text { Patton and Miller (1973) }\end{array}$ \\
\hline & Kobuk-Alatna Hills & \begin{tabular}{|l|} 
Hamilton (1984) \\
Patton (1973) \\
Patton and Miller (1973) \\
\end{tabular} \\
\hline Charley River & Tintina & $\begin{array}{l}\text { Dover and Miyaoka (1988) } \\
\text { Patton (1973) }\end{array}$ \\
\hline
\end{tabular}

\footnotetext{
${ }^{1}$ Complete reference citations are given in Appendix 2.
} 


\begin{tabular}{|c|c|c|}
\hline Quadrangle & Fault & Reference \\
\hline Circle & Tintina & $\begin{array}{l}\text { Foster and others (1983) } \\
\text { Patton (1973) } \\
\text { Weber and Foster (1982) } \\
\text { Yeend (1989) } \\
\end{array}$ \\
\hline \multirow[t]{3}{*}{ Cordova } & Etches & $\begin{array}{l}\text { Nelson and others (1985) } \\
\text { Winkler and Plafker (1981) }\end{array}$ \\
\hline & Ragged Mountain & $\begin{array}{l}\text { Kachadoorian (1960) } \\
\text { Nelson and others (1985) } \\
\text { Tysdal and others (1976) } \\
\text { Winkler and Plafker (1981) }\end{array}$ \\
\hline & Rude River & Winkler and Plafker (1981) \\
\hline Eagle & Tintina & \begin{tabular}{|l|} 
Foster (1976) \\
Patton (1973) \\
\end{tabular} \\
\hline \multirow[t]{2}{*}{ Fairbanks } & Minto & $\begin{array}{l}\text { Péwé and others (1966) } \\
\text { Puplan (1988) } \\
\text { Reger (1987a) }\end{array}$ \\
\hline & (liquefaction) & Combellick (1984) \\
\hline \multirow[t]{2}{*}{ Healy } & Healy & Thorson (1979) \\
\hline & Healy Creek & $\begin{array}{l}\text { Brogan and others (1975) } \\
\text { Wahrhaftig (1970a) }\end{array}$ \\
\hline Hughes & Kobuk-Alatna Hills & $\begin{array}{l}\text { Hamilton (1984) } \\
\text { Patton (1973) } \\
\text { Patton and Miller (1966) } \\
\end{array}$ \\
\hline Iditarod & Iditarod-Nixon Fork & $\begin{array}{l}\text { Bundtzen and Laird (1982, } \\
\text { 1983A) } \\
\text { Bundtzen and others (1988, } \\
\text { 1992) } \\
\text { Cady and others (1955) } \\
\text { Miller and Bundtzen (1988, } \\
\text { 1994) }\end{array}$ \\
\hline Iliamna & Bruin Bay & Magoon and others (1976) \\
\hline Juneau & Chatham Strait & Plafker and others (1978) \\
\hline
\end{tabular}


Appendix 3. (cont'd.)

\begin{tabular}{|c|c|c|}
\hline Quadrangle & Fault & Reference \\
\hline \multirow[t]{2}{*}{ Kateel River } & Huslia & $\begin{array}{l}\text { Brogan and others (1975) } \\
\text { Patton (1966) }\end{array}$ \\
\hline & Shoestring Dune & $\begin{array}{l}\text { Brogan and others (1975) } \\
\text { Patton (1966) } \\
\text { Weber and Péwé (1970) } \\
\end{array}$ \\
\hline Lake Clark & Lake Clark & $\begin{array}{l}\text { Magoon and others (1976) } \\
\text { Nelson and others (1983) }\end{array}$ \\
\hline Lime Hills & Denali-Farewell & $\begin{array}{l}\text { DGGS (unpub. data) } \\
\text { Gilbert (1981) }\end{array}$ \\
\hline \multirow[t]{2}{*}{ McGrath } & Denali-Farewell & $\begin{array}{l}\text { Blodgett and Gilbert (1983) } \\
\text { Bundtzen and others (1982) } \\
\text { DGGS (unpub. data) } \\
\text { Fernald (1960) } \\
\text { Gilbert (1981) } \\
\text { Gilbert and others (1990) } \\
\text { Plafker and others (1977) }\end{array}$ \\
\hline & Iditarod-Nixon Fork & Miller and Bundtzen (1988) \\
\hline Medfra & Iditarod-Nixon Fork & $\begin{array}{l}\text { Fernald (1960) } \\
\text { Miller and Bundtzen (1988) } \\
\text { Patton and others (1980) }\end{array}$ \\
\hline \multirow[t]{2}{*}{ Melozitna } & Huslia & Patton and others (1978) \\
\hline & Kaltag & \\
\hline Mount Katmai & Bruin Bay & Magoon and others (1976) \\
\hline Mount McKinley & Denali & $\begin{array}{l}\text { Plafker and others (1977) } \\
\text { Reed (1961) }\end{array}$ \\
\hline Norton Bay & Kaltag & $\begin{array}{l}\text { Cass (1959a) } \\
\text { Patton (1973) } \\
\text { Patton and Hoare (1968) }\end{array}$ \\
\hline Nulato & Kaltag & \begin{tabular}{|l|} 
Cass (1959b) \\
Patton (1973) \\
Patton and Hoare (1968) \\
Weber and Péwé (1970)
\end{tabular} \\
\hline & & \\
\hline
\end{tabular}




\begin{tabular}{|c|c|l|}
\hline Quadrangle & \multicolumn{1}{|c|}{ Fault } & \multicolumn{1}{|c|}{ Reference } \\
\hline Ruby & Kaltag & Patton (1973) \\
Patton and Hoare (1968)
\end{tabular}


Appendix 3. (cont'd.)

\begin{tabular}{|c|c|c|}
\hline Quadrangle & Fault & Reference \\
\hline \multirow[t]{4}{*}{ Talkeetna } & Chelatna Lake & $\begin{array}{l}\text { Nelson and Reed (1978) } \\
\text { Reed and Nelson (1980) }\end{array}$ \\
\hline & Denali & $\begin{array}{l}\text { Plafker and others (1977) } \\
\text { Reed and Nelson (1980) }\end{array}$ \\
\hline & Moose Creek & $\begin{array}{l}\text { Nelson and Reed (1978) } \\
\text { Reed and Nelson (1980 }\end{array}$ \\
\hline & Pingston Creek & $\begin{array}{l}\text { Nelson and Reed (1978) } \\
\text { Reed and Nelson (1980) }\end{array}$ \\
\hline Tanana & Kaltag & $\begin{array}{l}\text { Brogan and others (1975) } \\
\text { Chapman and others (1982) }\end{array}$ \\
\hline \multirow[t]{3}{*}{ Taylor Mountains } & Atsaksovluk & Cady and others (1955) \\
\hline & Boss & Cady and others (1955) \\
\hline & Holitna & $\begin{array}{l}\text { Cady and others (1955) } \\
\text { Plafker and others (1977) }\end{array}$ \\
\hline \multirow[t]{3}{*}{ Tyonek } & Bruin Bay & $\begin{array}{l}\text { Detterman and others (1975, } \\
1976) \\
\text { Hackett (1977) } \\
\text { Magoon and others (1976) }\end{array}$ \\
\hline & Castle Mountain & $\begin{array}{l}\text { Detterman and others (1974, } \\
\text { 1976) } \\
\text { Hackett (1977) } \\
\text { Magoon and others (1976) } \\
\text { Reger and Updike (1983) } \\
\text { Schmoll and others (1981) }\end{array}$ \\
\hline & Lake Clark & $\begin{array}{l}\text { Detterman and others (1976) } \\
\text { Hackett (1977) } \\
\text { Magoon and others (1976) } \\
\text { Schmoll and others (1981) }\end{array}$ \\
\hline Unalakleet & Kaltag & $\begin{array}{l}\text { Fisher (1982) } \\
\text { Patton (1973) } \\
\text { Patton and Hoare (1968) } \\
\text { Patton and Moll (1985) }\end{array}$ \\
\hline
\end{tabular}




\section{APPENDIX 4}

QUALITY OF MAP DATA IN TECHNICAL REFERENCES USED DURING GEOLOGIC EVALUATION OF PROPOSED ACCESS CORRIDORS IN ALASKA

\begin{tabular}{|c|c|c|c|}
\hline \multirow[t]{2}{*}{ Quadrangle } & \multirow[t]{2}{*}{ Reference $^{1}$} & \multicolumn{2}{|c|}{ Data Quality } \\
\hline & & Bedrock Geology & Surficial Geology \\
\hline Ambler River & $\begin{array}{c}\text { Hamilton (1984) } \\
\text { Pessel and Brosgé (1977) }\end{array}$ & $\overline{3}$ & 3 \\
\hline Anchorage & Reger $(1978,1981 \mathrm{a}, \mathrm{b})$ & $\begin{array}{l}- \\
- \\
-\end{array}$ & $\begin{array}{l}5 \\
5 \\
5\end{array}$ \\
\hline Baird Mountains & $\begin{array}{c}\text { Karl and others (1990) } \\
\text { Patton and Miller (1968) } \\
\text { Pinney (1992 photointerpretation) }\end{array}$ & $\begin{array}{l}3 \\
3 \\
-\end{array}$ & $\begin{array}{l}- \\
2 \\
3\end{array}$ \\
\hline Barrow & Williams and Carter (1984) & - & 3 \\
\hline Barter Island & Pinney (1992 photointerpretation) & - & 3 \\
\hline Beechey Point & $\begin{array}{c}\text { Hickmott (1986) } \\
\text { Rawlinson }(1986 \mathrm{a}, \mathrm{b}, \mathrm{c})\end{array}$ & $\begin{array}{l}5 \\
5 \\
- \\
5\end{array}$ & $\begin{array}{l}5 \\
5 \\
5 \\
5\end{array}$ \\
\hline Bendeleben & $\begin{array}{c}\text { Kaufman (1986) } \\
\text { Till and others (1986) }\end{array}$ & $\overline{-}$ & $\begin{array}{l}3 \\
-\end{array}$ \\
\hline Bering Glacier & Miller $(1961,1971)$ & $\begin{array}{l}3 \\
3\end{array}$ & $\begin{array}{l}3 \\
3\end{array}$ \\
\hline Bethel & Hoare and Coonrad (1959) & 2 & 2 \\
\hline Bettles & $\begin{array}{c}\text { Patton and Miller (1973) } \\
\text { Reger (1992 photointerpretation) }\end{array}$ & $\begin{array}{l}3 \\
-\end{array}$ & $\overline{3}$ \\
\hline Black River & Brabb (1970) & 2 & 2 \\
\hline Bradfield Canal & $\begin{array}{c}\text { Gehrels and Berg (1984) } \\
\text { Pinney (1992 photointpretation) }\end{array}$ & 1 & - \\
\hline
\end{tabular}

\footnotetext{
${ }^{1}$ Complete reference citations are given in Appendix 2.
} 


\begin{tabular}{|c|c|c|c|}
\hline \multirow[t]{2}{*}{ Quadrangle } & \multirow[t]{2}{*}{ Reference } & \multicolumn{2}{|c|}{ Data Quality } \\
\hline & & Bedrock Geology & Surficial Geology \\
\hline Candle & Patton (1967) & 3 & 2 \\
\hline Chandalar & $\begin{array}{c}\text { Brosgé and Reiser (1964) } \\
\text { Hamilton (1978) }\end{array}$ & 3 & $\overline{-}$ \\
\hline Chandler Lake & $\begin{array}{l}\text { Hamilton (1979) } \\
\text { Kelley (1990) }\end{array}$ & - & $\begin{array}{l}3 \\
-\end{array}$ \\
\hline Charley River & Dover and Miyaoka (1988) & 3 & 3 \\
\hline Chignik & Detterman and others $(1981 \mathrm{a}, \mathrm{b})$ & $\begin{array}{l}3 \\
-\end{array}$ & - \\
\hline Circle & Foster and others (1983) & 3 & 3 \\
\hline Cold Bay & $\begin{array}{l}\text { DuBois and others (1989) } \\
\text { McLean and others (1978) } \\
\text { Waldron (1961) }\end{array}$ & $\begin{array}{l}3 \\
3 \\
3\end{array}$ & $\begin{array}{l}- \\
2 \\
2\end{array}$ \\
\hline Cordova & $\begin{array}{c}\text { Kachadoorian (1960) } \\
\text { Winkler and Plafker (1981) }\end{array}$ & $\overline{3}$ & $\begin{array}{l}5 \\
2\end{array}$ \\
\hline $\begin{array}{c}\text { DeLong } \\
\text { Mountains }\end{array}$ & $\begin{array}{l}\text { Ellersieck and others (1990) } \\
\text { Mayfield and others (1990) } \\
\text { Mull (unpub. data) } \\
\text { Sable and others (1984) } \\
\text { Sable and Mangus (1984) }\end{array}$ & $\begin{array}{l}5 \\
5 \\
3 \\
5 \\
5\end{array}$ & $\begin{array}{l}3 \\
3 \\
2 \\
4 \\
4\end{array}$ \\
\hline $\begin{array}{l}\text { Demarcation } \\
\text { Point }\end{array}$ & Reiser and others (1980) & - & 3 \\
\hline Dillingham & $\begin{array}{c}\text { Beikman (1974) } \\
\text { Mertie (1938) } \\
\text { Reger (1992 photointerpretation) }\end{array}$ & $\begin{array}{l}1 \\
2 \\
-\end{array}$ & $\begin{array}{l}- \\
-\end{array}$ \\
\hline Eagle & Foster (1976) & 3 & 3 \\
\hline Fairbanks & Péwé and others (1966) & $\begin{array}{l}3 \\
- \\
4 \\
-\end{array}$ & $\begin{array}{l}3 \\
4 \\
4 \\
4\end{array}$ \\
\hline
\end{tabular}




\begin{tabular}{|c|c|c|c|}
\hline \multirow[t]{2}{*}{ Quadrangle } & \multirow[t]{2}{*}{ Reference } & \multicolumn{2}{|c|}{ Data Quality } \\
\hline & & Bedrock Geology & Surficial Geology \\
\hline Flaxman Island & Carter and others (1986) & - & 3 \\
\hline Harrison Bay & $\begin{array}{l}\text { Carter and Galloway (1985) } \\
\text { Rawlinson (1990) }\end{array}$ & $\begin{array}{l}3 \\
5\end{array}$ & $\begin{array}{l}3 \\
5\end{array}$ \\
\hline Healy & $\begin{array}{l}\text { Csejtey and others (1986) } \\
\text { Gilbert and Bundtzen (1976) } \\
\text { Wahrhaftig }(1970 a, b)\end{array}$ & $\begin{array}{l}3 \\
4 \\
5 \\
5\end{array}$ & $\begin{array}{l}3 \\
- \\
5 \\
5\end{array}$ \\
\hline Holy Cross & $\begin{array}{c}\text { Beikman (1974) } \\
\text { Livingston (1983) } \\
\text { Livingston (1992 } \\
\text { photointerpretation) }\end{array}$ & $\begin{array}{l}1 \\
- \\
-\end{array}$ & $\begin{array}{l}- \\
5 \\
3\end{array}$ \\
\hline Howard Pass & $\begin{array}{l}\text { Hamilton (1984) } \\
\text { Mull (unpub. data) }\end{array}$ & - & $\begin{array}{l}3 \\
-\end{array}$ \\
\hline Hughes & $\begin{array}{c}\text { Patton and Miller (1966) } \\
\text { Reger (1992 photointerpretation) }\end{array}$ & $\begin{array}{l}3 \\
-\end{array}$ & $\begin{array}{l}2 \\
3\end{array}$ \\
\hline Iditarod & $\begin{array}{c}\text { Bundtzen and Laird }(1982,1983) \\
\text { Bundtzen and others }(1988,1992) \\
\text { Miller and Bundtzen }(1994) \\
\text { Reger (1992 photointerpretation) }\end{array}$ & $\begin{array}{l}5 \\
5 \\
5 \\
5 \\
3 \\
-\end{array}$ & $\begin{array}{l}5 \\
5 \\
5 \\
5 \\
- \\
3\end{array}$ \\
\hline Ikpikpuk River & Carter and Galloway (1988) & 2 & 3 \\
\hline Iliamna & Detterman and Reed $(1973,1980)$ & - & $\begin{array}{l}3 \\
-\end{array}$ \\
\hline Juneau & $\begin{array}{c}\text { Beikman (1975) } \\
\text { Pinney (1992 photointerpretation) }\end{array}$ & $\begin{array}{l}1 \\
-\end{array}$ & - \\
\hline Kantishna River & $\begin{array}{c}\text { Chapman and others }(1975 \mathrm{a}, \mathrm{b}) \\
\text { Collins }(1985)\end{array}$ & $\begin{array}{l}3 \\
3 \\
-\end{array}$ & $\begin{array}{l}3 \\
3 \\
2\end{array}$ \\
\hline Karluk & Detterman and others (1987) & 3 & 3 \\
\hline Kateel River & Patton (1966) & 3 & 2 \\
\hline
\end{tabular}




\begin{tabular}{|c|c|c|c|}
\hline \multirow[t]{2}{*}{ Quadrangle } & \multirow{2}{*}{ Reference } & \multicolumn{2}{|c|}{ Data Quality } \\
\hline & & Bedrock Geology & Surficial Geology \\
\hline Killik River & $\begin{array}{l}\text { Chapman and others (1964) } \\
\text { Hamilton (1980) }\end{array}$ & $\begin{array}{l}3 \\
-\end{array}$ & $\overline{3}$ \\
\hline Lake Clark & $\begin{array}{c}\text { Nelson and others (1983) } \\
\text { Reger (1992 photointerpretation) }\end{array}$ & $\begin{array}{l}3 \\
-\end{array}$ & $\overline{-}$ \\
\hline Lime Hills & $\begin{array}{c}\text { Capps (1930) } \\
\text { DGGS (unpub. data) } \\
\text { Gilbert (1981) } \\
\text { Gilbert and others (1990) } \\
\text { Reger (1992 photointerpretation) } \\
\text { Reger (1992 satellite-imagery } \\
\text { interpretation) } \\
\text { Weber (1961) }\end{array}$ & $\begin{array}{l}2 \\
3 \\
5 \\
5 \\
- \\
- \\
3\end{array}$ & $\begin{array}{l}- \\
- \\
5 \\
5 \\
3 \\
2 \\
3\end{array}$ \\
\hline Livengood & Chapman and others (1971) & 3 & 2 \\
\hline Lookout Ridge & Yeend (1983) & 2 & 3 \\
\hline McGrath & $\begin{array}{c}\text { Blodgett and Gilbert (1983) } \\
\text { Bundtzen and others (1982) } \\
\text { Bundtzen and Laird (1983) } \\
\text { DGGS (unpub. data) } \\
\text { Fernald (1960) } \\
\text { Gilbert (1981) } \\
\text { Gilbert and others }(1988,1990) \\
\text { Kline and others (1986) } \\
\text { Reed and Elliott (1970) } \\
\text { Weber (1961) }\end{array}$ & $\begin{array}{l}5 \\
5 \\
5 \\
3 \\
- \\
5 \\
5 \\
5 \\
5 \\
2 \\
-\end{array}$ & $\begin{array}{l}- \\
5 \\
5 \\
2 \\
2 \\
5 \\
5 \\
5 \\
5 \\
- \\
3\end{array}$ \\
\hline Meade River & Williams (1983) & 3 & 3 \\
\hline Medfra & $\begin{array}{c}\text { Fernald (1960) } \\
\text { Patton and others (1980) }\end{array}$ & $\begin{array}{l}2 \\
2\end{array}$ & $\begin{array}{l}3 \\
2\end{array}$ \\
\hline Melozitna & $\begin{array}{c}\text { Patton and others (1978) } \\
\text { Reger (1992 photointerpretation) }\end{array}$ & $\begin{array}{l}3 \\
-\end{array}$ & $\begin{array}{l}2 \\
3\end{array}$ \\
\hline $\begin{array}{l}\text { Misheguk } \\
\text { Mountain }\end{array}$ & $\begin{array}{l}\text { Sable and Mangus (1984) } \\
\text { Sable and others }(1984 a, b)\end{array}$ & $\begin{array}{l}5 \\
5 \\
5\end{array}$ & $\begin{array}{l}5 \\
5 \\
5\end{array}$ \\
\hline
\end{tabular}




\begin{tabular}{|c|c|c|c|}
\hline \multirow[t]{2}{*}{ Quadrangle } & \multirow[t]{2}{*}{ Reference } & \multicolumn{2}{|c|}{ Data Quality } \\
\hline & & Bedrock Geology & Surficial Geology \\
\hline Mount Katmai & Riehle and others (1987) & 3 & 3 \\
\hline Mount McKinley & $\begin{array}{c}\text { Bundtzen and others (1976) } \\
\text { Reed (1961) } \\
\text { Ten Brink (1984) }\end{array}$ & $\begin{array}{l}5 \\
3 \\
-\end{array}$ & $\begin{array}{l}- \\
3 \\
4\end{array}$ \\
\hline $\begin{array}{c}\text { Mount } \\
\text { Michelson }\end{array}$ & $\begin{array}{l}\text { Carter and others (1986) } \\
\text { Rawlinson (1990) }\end{array}$ & $\begin{array}{l}- \\
-\end{array}$ & $\begin{array}{l}3 \\
5\end{array}$ \\
\hline Naknek & $\begin{array}{c}\text { Ottley (1982) } \\
\text { Reger (1992 photointerpretation) } \\
\text { Riehle and others (1987) }\end{array}$ & $\begin{array}{l}- \\
- \\
3\end{array}$ & $\begin{array}{l}5 \\
3 \\
-\end{array}$ \\
\hline Noatak & $\begin{array}{c}\text { Beikman and Lathram (1976) } \\
\text { Krause (1985) } \\
\text { Pinney (1992 photointerpretation) }\end{array}$ & $\begin{array}{l}1 \\
1 \\
-\end{array}$ & $\begin{array}{l}- \\
2 \\
3\end{array}$ \\
\hline Norton Bay & $\begin{array}{c}\text { Cass (1959) } \\
\text { Riehle and others (1981) }\end{array}$ & $\begin{array}{l}2 \\
-\end{array}$ & $\begin{array}{l}2 \\
4\end{array}$ \\
\hline Nulato & $\begin{array}{c}\text { Bickel and Patton (1957) } \\
\text { Cass (1959) } \\
\text { Martinson (1982) }\end{array}$ & $\begin{array}{l}3 \\
3 \\
-\end{array}$ & $\begin{array}{l}2 \\
2 \\
-\end{array}$ \\
\hline Ophir & $\begin{array}{l}\text { Bundtzen and Laird (1980) } \\
\text { Chapman and others (1985) }\end{array}$ & $\begin{array}{l}5 \\
3\end{array}$ & $\begin{array}{l}5 \\
3\end{array}$ \\
\hline Petersburg & Brew and others (1984) & 3 & 2 \\
\hline $\begin{array}{l}\text { Philip Smith } \\
\text { Mountains }\end{array}$ & $\begin{array}{c}\text { Brosgé and others (1979) } \\
\text { Hamilton (1978) }\end{array}$ & $\begin{array}{l}3 \\
-\end{array}$ & $\overline{3}$ \\
\hline Port Moller & $\begin{array}{c}\text { Reger (1992 satelline-imagery } \\
\text { interpretation } \\
\text { Wilson and others (1991) }\end{array}$ & 3 & 2 \\
\hline Ruby & $\begin{array}{c}\text { Cass (1959) } \\
\text { Pinney (1992 photointerpretation) }\end{array}$ & $\begin{array}{l}2 \\
-\end{array}$ & $\overline{3}$ \\
\hline Russian Mission & $\begin{array}{l}\text { Bundtzen and Laird (1991) } \\
\text { Hoare and Coonrad (1959b) }\end{array}$ & $\begin{array}{l}5 \\
2\end{array}$ & $\begin{array}{l}5 \\
2\end{array}$ \\
\hline
\end{tabular}




\begin{tabular}{|c|c|c|c|}
\hline \multirow[t]{2}{*}{ Quadrangle } & \multirow[t]{2}{*}{ Reference } & \multicolumn{2}{|c|}{ Data Quality } \\
\hline & & Bedrock Geology & Surficial Geology \\
\hline Sagavanirktok & $\begin{array}{c}\text { Beikman (1976) } \\
\text { Ferrians (1971) } \\
\text { Pinney (1992 photointerpretation) } \\
\text { Yeend (1971) }\end{array}$ & $\begin{array}{l}1 \\
3 \\
- \\
3\end{array}$ & $\begin{array}{l}- \\
3 \\
3 \\
3\end{array}$ \\
\hline Selawik & Patton and Miller (1968) & 3 & 2 \\
\hline Shungnak & $\begin{array}{c}\text { Fernald (1964) } \\
\text { Fritts }(1969,1970) \\
\text { Hitzman and others (1982) } \\
\text { Pallister and Carlson (1988) } \\
\text { Patton and others (1968) }\end{array}$ & $\begin{array}{l}- \\
5 \\
5 \\
3 \\
5 \\
3\end{array}$ & $\begin{array}{l}3 \\
- \\
- \\
- \\
- \\
2\end{array}$ \\
\hline Sleetmute & $\begin{array}{c}\text { Decker and others (1984) } \\
\text { Miller and others (1989) } \\
\text { Reger (1992 photointerpretation) } \\
\text { Reifenstuhl and others (1984) } \\
\text { Robinson and others (1984) } \\
\text { Waythomas (1990) }\end{array}$ & $\begin{array}{l}5 \\
3 \\
- \\
5 \\
5 \\
-\end{array}$ & $\begin{array}{l}- \\
3 \\
3 \\
- \\
- \\
5\end{array}$ \\
\hline Solomon & $\begin{array}{c}\text { Kaufman (1986) } \\
\text { Till and others (1986) }\end{array}$ & $\overline{3}$ & $\begin{array}{l}3 \\
-\end{array}$ \\
\hline Survey Pass & $\begin{array}{c}\text { Hamilton (1981) } \\
\text { Nelson Grybeck (1980) }\end{array}$ & - & $\begin{array}{l}3 \\
-\end{array}$ \\
\hline Taku River & $\begin{array}{c}\text { Beikman (1975) } \\
\text { Brew and Ford (1985) } \\
\text { Pinney (1992 photointerpretation) }\end{array}$ & $\begin{array}{l}1 \\
3 \\
-\end{array}$ & $\begin{array}{l}- \\
- \\
3\end{array}$ \\
\hline Talkeetna & $\begin{array}{l}\text { Nelson and Reed (1978) } \\
\text { Reed and Nelson (1980) }\end{array}$ & $\overline{3}$ & $\begin{array}{l}3 \\
2\end{array}$ \\
\hline Tanana & Chapman and others (1982) & 3 & 3 \\
\hline $\begin{array}{c}\text { Taylor } \\
\text { Mountains }\end{array}$ & $\begin{array}{c}\text { Beikman (1974) } \\
\text { Cady and others (1955) } \\
\text { DGGS (unpub. data) } \\
\text { Reger (1992 photointerpretation) }\end{array}$ & $\begin{array}{l}1 \\
2 \\
3 \\
-\end{array}$ & $\begin{array}{l}- \\
- \\
2 \\
3\end{array}$ \\
\hline Teshekpuk & Carter (1983) & - & 3 \\
\hline
\end{tabular}




\begin{tabular}{|c|c|c|c|}
\hline \multirow[t]{2}{*}{ Quadrangle } & \multirow[t]{2}{*}{ Reference } & \multicolumn{2}{|c|}{ Data Quality } \\
\hline & & Bedrock Geology & Surficial Geology \\
\hline Tyonek & $\begin{array}{c}\text { Barnes (1966) } \\
\text { Detterman and others }(1974,1976) \\
\text { Magoon and others }(1976) \\
\text { Odum and others }(1988) \\
\text { Rawlinson and others (1982a, b, c) } \\
\text { Reger (1978) } \\
\text { Reger and Updike (1983) } \\
\text { Schmoll and Yehle (1987) } \\
\text { Solie and others (1991) } \\
\text { Weber (1961) }\end{array}$ & $\begin{array}{l}5 \\
- \\
3 \\
3 \\
- \\
- \\
- \\
- \\
- \\
- \\
5 \\
5 \\
-\end{array}$ & $\begin{array}{l}- \\
3 \\
- \\
- \\
3 \\
5 \\
5 \\
5 \\
5 \\
3 \\
5 \\
5 \\
3\end{array}$ \\
\hline Ugashik & Detterman and others (1987) & 3 & 3 \\
\hline Umiat & Carter and Galloway (1986) & 2 & 3 \\
\hline Unalakleet & $\begin{array}{c}\text { Cass (1959) } \\
\text { Patton and Moll (1985) } \\
\text { Riehle and others (1981) }\end{array}$ & $\begin{array}{l}2 \\
3 \\
-\end{array}$ & $\begin{array}{l}2 \\
2 \\
4\end{array}$ \\
\hline Utukok River & Yeend (1984) & 2 & 3 \\
\hline Wiseman & $\begin{array}{l}\text { Dillon and others (1986) } \\
\text { Hamilton (1979) }\end{array}$ & $\begin{array}{l}3 \\
-\end{array}$ & $\overline{3}$ \\
\hline
\end{tabular}

